\title{
Group-III-nitride and halide-perovskite semiconductor gain media for amplified spontaneous emission and lasing applications
}

\author{
Tien Khee $\mathrm{Ng}^{1}\left(\mathbb{D}\right.$, Jorge A Holguin-Lerma ${ }^{1}\left(\mathbb{D}\right.$, Chun Hong Kang ${ }^{1}(\mathbb{D}$, \\ Islam Ashry $\mathbb{D}$, Huafan Zhang $\mathbb{D}$, Giada Bucci and Boon S Ooi \\ Photonics Laboratory, Computer, Electrical and Mathematical Sciences and Engineering (CEMSE), \\ King Abdullah University of Science and Technology (KAUST), Thuwal 21534, Saudi Arabia \\ E-mail: tienkhee.ng@kaust.edu.sa and boon.ooi@kaust.edu.sa
}

Received 28 April 2020, revised 28 September 2020

Accepted for publication 23 December 2020

Published 22 January 2021

\begin{abstract}
Group-III-nitride optical devices are conventionally important for displays and solid-state lighting, and recently have garnered much interest in the field of visible-light communication. While visible-light laser technology has become mature, developing a range of compact, small footprint, high optical power components for the green-yellow gap wavelengths still requires material development and device design breakthroughs, as well as hybrid integration of materials to overcome the limitations of conventional approaches. The present review focuses on the development of laser and amplified spontaneous emission (ASE) devices in the visible wavelength regime using primarily group-III-nitride and halide-perovskite semiconductors, which are at disparate stages of maturity. While the former is well established in the violet-blue-green operating wavelength regime, the latter, which is capable of solution-based processing and wavelength-tunability in the green-yellow-red regime, promises easy heterogeneous integration to form a new class of hybrid semiconductor light emitters. Prospects for the use of perovskite in ASE and lasing applications are discussed in the context of facile fabrication techniques and promising wavelength-tunable light-emitting device applications, as well as the potential integration with group-III-nitride contact and distributed Bragg reflector layers, which is promising as a future research direction. The absence of lattice-matching limitations, and the presence of direct bandgaps and excellent carrier transport in halide-perovskite semiconductors, are both encouraging and thought-provoking for device researchers who seek to explore new possibilities either experimentally or theoretically. These
\end{abstract}

Authors contributed equally. 
combined properties inspire researchers who seek to examine the suitability of such materials for potential novel electrical injection devices designed for targeted applications related to lasing and operating-wavelength tuning.

Keywords: group-III nitride, halide perovskite, laser, amplified spontaneous emission, superluminescent diode

(Some figures may appear in colour only in the online journal)

\section{Introduction}

\subsection{Group-III-nitride semiconductors}

Group-III-nitride materials have been of significant interest to researchers and the industrial community for decades. The various versatile properties of these materials have led to broad interest for solid-state lighting, power electronics, and energy conversion devices, such as light-emitting diodes (LEDs) and lasers [1-4]; high-electron-mobility transistors [5, 6]; photodetectors [7]; solar cells; and photoelectrochemical [8] devices.

1.1.1. Material properties. Three binary group-III-nitride crystalline structures (i.e. InN, AlN, and GaN) exist physically: wurtzite, zinc blende, and rock salt, as shown in figure 1 [9]. Wurtzite structures exhibit better thermodynamic stability at ambient temperature and pressure than the latter two lattice structures [1]. Moreover, because of the large ionicities of the chemical bonds in wurtzite structures, group-III nitrides exhibit chemical and thermal stabilities that are superior to those of other covalent group-III-V compounds (i.e. InP, $\mathrm{GaAs}$, and $\mathrm{GaSb}$ ), and are thus suitable for use in harsh environments $[1,10]$.

However, the large ionicities of metal-nitride bonds and non-centrosymmetric atomic arrangements along the $c$-axis cause spontaneous polarization in wurtzite group-III nitrides [11-13]. These polarization fields can increase drift velocities and interfacial charges at heterojunctions [14-16]. This must be considered when designing optoelectronic and electronic devices.

As essential semiconductor materials, the group-III nitrides exhibit direct bandgap characteristics that allow effective radiative recombination and photon emission [1, 10]. The bandgaps of the binary compounds $\mathrm{AlN}, \mathrm{GaN}$, and $\mathrm{InN}$ are $\sim 6.11 \mathrm{eV}, \sim 3.42 \mathrm{eV}$, and $\sim 0.65 \mathrm{eV}$, respectively $[17,18]$. The bandgap of InN was initially accepted as $1.9 \mathrm{eV}$ [19] until several pivotal experimental verifications eventually adjusted the value to $\sim 0.65 \mathrm{eV}$. The new value was recognized after 2002 [18, 20, 21]. Simply tuning the group-III element composition (i.e. In, $\mathrm{Ga}$, and $\mathrm{Al}$ ) in ternary compounds allows the emission wavelengths of these materials at room temperature to provide broad coverage of the optical spectrum, i.e. from the deep-ultraviolet $(\sim 200 \mathrm{~nm})$ to the infrared spectrum $(\sim 1771 \mathrm{~nm})$, as shown in figure 2 . This wavelength selection flexibility has inspired a wide range of optoelectronics, e.g. visible-wavelength LEDs and lasers, which eventually led to the development of deep-ultraviolet devices.
1.1.2. Material growth and other important issues. Hydride vapor phase epitaxy (HVPE) is an important technique used to grow single-crystal group-III nitride layers during their early development $[22,23]$. The first $\mathrm{GaN}$ violet emitter was grown via this technique in the 1970s [24-26]. This technique has exhibited high growth rates and repeatable growth of relatively high-quality group-III nitrides. However, p-type dopant incorporation has remained an issue. After substantial growth optimization and device development, HVPE has been used for the production of group-III nitride bulk layers [27] and free-standing substrates [28,29] since the 1990s, mainly because it offers high growth rates $\left(>100 \mu \mathrm{m} \mathrm{hr}^{-1}\right)$.

Later, metal-organic chemical vapor deposition (MOCVD), also known as metal-organic vapor phase epitaxy, attracted substantial attention because of its eventual success in p-type doping of group-III nitrides [30] and the invention of the first blue LED [31]. Since then, MOCVD has become an industrial workhorse for the epitaxial growth of group-III nitrides. It typically operates at high temperatures and moderate pressures (10 Torr-760 Torr) and uses metal-organic precursors. The growth rates are relatively fast and can be controlled via chemical flows. There are reports of growth of various nanostructures [32], e.g. quantum dots (QDs) [33] and nanowires [34], as well as their subsequent implementations as LEDs or lasers [35]. Due to precursor availability, high growth rates, and growth technique scalability, they are appropriate for commercial mass production. To increase the In composition, the growth temperature and pressure must be lowered [36]. However, growing high-quality, In-rich $\mathrm{InGaN}$ is challenging because of the low thermodynamic stability and high evaporation rate of $\mathrm{InN}$ at high temperatures. This is the cause for the lack of green-yellow emitters for commercial solid-state lighting applications, which continues to exist despite some indications of research success [37-40] and even the pursuit of MOCVD-fabricated $>630 \mathrm{~nm}$ GaN LEDs [41, 42].

In addition to the aforementioned chemical vapor deposition (CVD) techniques, physical vapor deposition techniques, especially molecular beam epitaxy (MBE), represent another group of established epitaxial growth methods used in research. One variant of the MBE growth technique uses ammonia gas as a group- $\mathrm{V}$ source in high growth rate implementations [43-46]. A nitrogen-plasma-assisted MBE system uses high-purity group-III metallic sources and a radio-frequency (RF) nitrogen plasma as a group-V source. Unlike MOCVD or ammonia-assisted MBE, nitrogen-plasmaassisted MBE growth can be performed in high vacuum environments. This provides much lower growth rates but excellent atomic layer arrangement controllability and high 


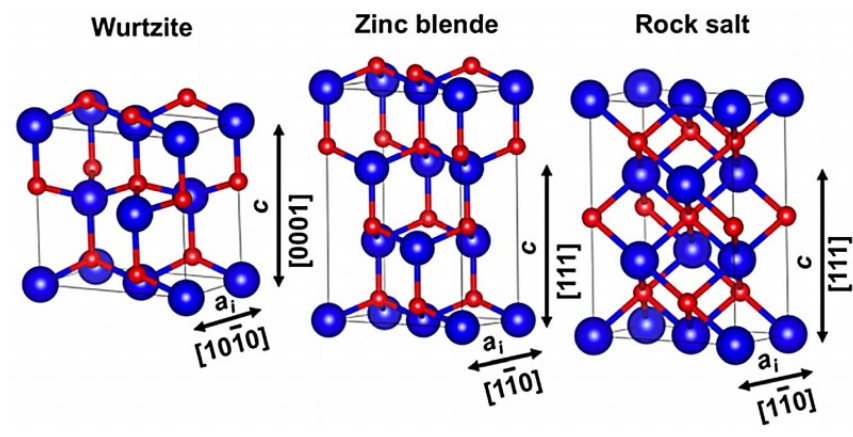

Figure 1. Three basic group-III-nitride crystal structures. Adapted from [9], with permission of AIP publishing.

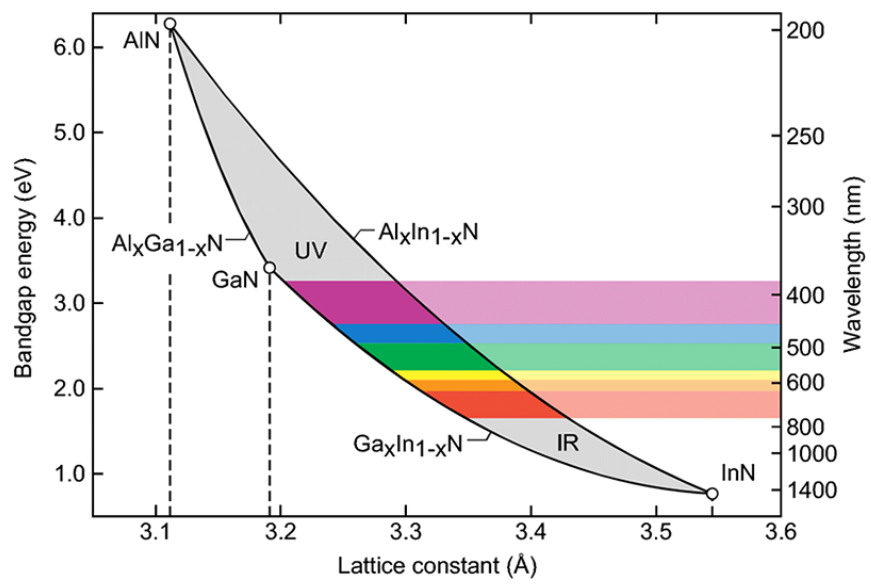

Figure 2. Tunable group-III-nitride ternary alloy bandgaps. Reproduced with permission from [11]. (C) Cambridge University Press. Reprinted with permission.

semiconductor crystal quality. Thus, it can be utilized to grow nanostructures such as QDs [47, 48] and nanowires [4, 49], as well as to grow monolayers such as quantum wells (QWs) [48] and distributed Bragg reflectors (DBRs) [50] in a precise manner. On the other hand, MBE can be performed at much lower temperatures than MOCVD. This can inhibit In desorption from the substrate surface and increase the In content while eliminating phase segregation [36].

Other growth techniques have also been reported, although these are not directly related to the epitaxial growth of laser structures. Their exploration has continued because of their potential to enable low-cost device application solutions. These techniques include reactive sputtering [51], thermal atomic layer deposition (ALD) [52], pulsed-laser deposition (PLD) [53], activated reactive evaporation (ARE) [54], and electrochemical solution syntheses [55]. Prabaswara et al [51] highlighted the technique of magnetron sputter epitaxy, which is a variant of reactive sputter deposition and a subset of the physical vapor deposition method. The technique was developed for high-vacuum $\mathrm{GaN}$ deposition, and has the potential for large-scale production of channel layers for thin-film transistors [56]. Banergee et al [52] drew a parallel between the potential of poly-GaN and the proven industrial application of poly-Si to solar cell devices. The researchers described a poly-GaN deposition based on the thermal
ALD process that used thermal reactions at $400{ }^{\circ} \mathrm{C}$, without cracking $\mathrm{NH}_{3}$ into $\mathrm{N}$ radicals using a plasma, hot-filament, or electron beam. Vispute et al [53] conducted PLD of GaN on dissimilar substrates for scientific evaluation. This technique is still being studied. Biju et al [54] reported a modified ARE method based on nitrogen plasmas and gallium evaporation that can be used to grow polycrystalline films. Kang et al [55] further demonstrated the electrochemical deposition of $\mathrm{GaN}$ on silicon using an aqueous solution that contained $\mathrm{Ga}\left(\mathrm{NO}_{3}\right)_{3}$ and $\mathrm{NH}_{4} \mathrm{NO}_{3}$. These examples further elucidate the motivation of the community to explore alternative deposition techniques for LED and non-LED applications and the direction towards low-cost, scalable production.

More recently, remote epitaxy using two-dimensional layers such as graphene has received substantial attention. For example, Jeong et al [57] reported the fabrication of deformable microrod LEDs and achieved substrate reuse. Zhao et al [4] and Min et al [58] further reported advances in nanowire and nanorod growth on unconventional substrates such as metal and quartz substrates.

Various low-dislocation-density planar epitaxial growth issues still remain [59].

1.1.2.1. Mismatch between epitaxy and substrates. One major concern is the substrate-epilayer mismatch in crystal structures, lattice constants, and thermal expansion coefficients. This leads to substantial challenges in selecting suitable substrates, managing structural defects, and manipulating polarization fields.

The growth of group-III nitrides is hindered by a lack of native substrates. Heteroepitaxial growth results in large mismatches between wurtzite group-III nitrides and conventional substrates such as silicon (111) and corundum sapphire (0001) ( $\alpha$-phase $\mathrm{Al}_{2} \mathrm{O}_{3}$ or $\left.\alpha-\mathrm{Al}_{2} \mathrm{O}_{3}\right)$. This introduces high edge- and screw-dislocation densities, as well as hexagonal macro defects and even cracks [2, 60]. While GaN maintains its hexagonal lattice and epitaxial relationship with $\mathrm{Si}$ (111) in a straightforward manner, the GaN hexagonal lattice is spontaneously rotated by $30^{\circ}$ when grown onto the trigonal lattice of sapphire (see figure 3) [61]. Interestingly, this allows the GaN lattice to align with the corresponding sapphire lattices and produces a $\sim 16 \%$ coincidental lattice mismatch instead of the $\sim 33 \%$ expected if we were to calculate the lattice mismatch value using the free-standing lattice constants of GaN and sapphire [62].

Differences in thermal expansion coefficients (see table 1) further result in dislocations and residual strain with regard to epitaxy. This contributes to the piezoelectric polarization fields in InGaN/GaN QWs, as well as to residual compressive and tensile strains in GaN grown on sapphire (0001) and Si (111), respectively (see figure 3). This can be measured using Raman backscattering spectroscopy or high-resolution $\mathrm{x}$-ray diffraction. We should be aware that in actual LED epitaxial growth, a low-temperature AlN buffer layer is grown on the substrates to enhance strain field interactions and annihilate dislocations, as well as to ensure the growth of metal-polar group-III nitrides. Nevertheless, mismatch values 
Lattice Mismatch

(a)
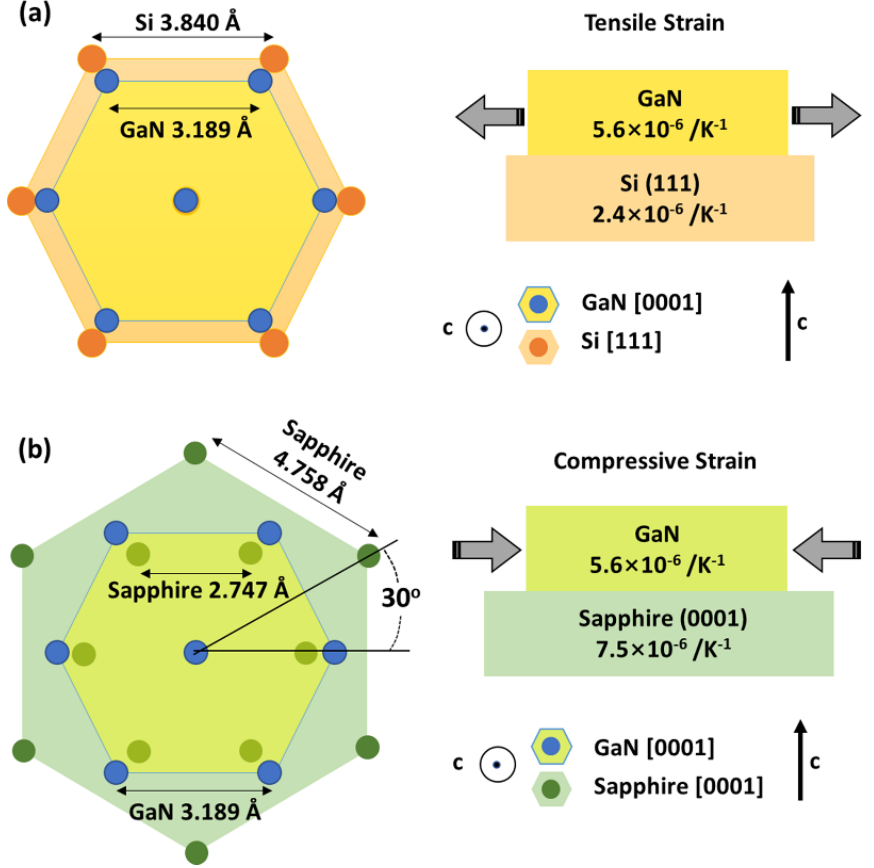

c $\begin{aligned} & \text { GaN [0001] } \\ & \text { Sapphire [0001] }\end{aligned} \mid$ c

Figure 3. Lattice and thermal mismatches of (a) GaN on $\mathrm{Si}$ (111) and (b) GaN on Sapphire (0001). GaN experiences tensile and compressive strains on the two substrates, respectively, due to thermal mismatch. These strains can be measured using Raman spectroscopy or x-ray diffraction. c denotes the (0001) crystal orientation or the Ga-polar orientation. Adapted by permission from Springer Nature Customer Service Centre GmbH: Springer Nature, Journal of the Korean Physical Society [62], () 2013.

Table 1. Considerations for $\mathrm{GaN}$ epitaxial growth on foreign substrates (data relative to the GaN properties) [63]. Republished with permission of the Institution of Engineering and Technology, from [63] permission conveyed through Copyright Clearance Center, Inc.

\begin{tabular}{lllll}
\hline Substrate & Bulk GaN & SiC & Sapphire & Silicon \\
\hline Lattice mismatch $^{\mathrm{a}}$ & None & $3.5 \%$ & $16 \%$ & $17 \%$ \\
Thermal mismatch & None & $+33 \%$ & $-25 \%$ & $+116 \%$
\end{tabular}

a Magnitude of lattice mismatch. Readers are referred to page 57 of [64] on definition and discussion on lattice mismatch and strain, as well as perpendicular lattice-mismatch.

remain excessively large and high-performance laser devices still require native substrates, i.e. GaN or potentially AlN.

To minimize the lattice mismatch between group-III nitride and substrate, $6 \mathrm{H}-\mathrm{SiC}$ was also studied. Devices grown on $\mathrm{SiC}$ have lower defect densities and exhibit relatively good performances compared to those grown on sapphire. Despite the high costs that almost inhibit the commercialization of these devices [50], 6H-SiC was adopted by Cree as the substrate of choice for LED production. Meanwhile, other efforts based on the use of sapphire substrates have demonstrated buffer layer insertion [27] and epitaxial lateral overgrowth techniques $[2,33]$, although the overall processes employed are often complex.

Although the free-standing native substrates are expensive and challenging with regard to substrate up-scaling, direct epitaxial growth on native substrates leads to highperformance lasers and superluminescent diodes. This will be discussed further in section 2.

Because of the economies of scale relevant to producing group-III-nitride LED devices, Si substrates can be used as an alternative platform for lowering the cost of production while enabling the integration with $\mathrm{Si}$ electronics through wafer bonding. However, the thermal expansion coefficient and lattice mismatches tend to cause cracking issues that degrade device performance. To tackle the problem, researchers have demonstrated group-III nitride epitaxial growth on $\mathrm{Si}$ substrates using GaN templates by MBE [36]. Another team carefully suppressed the wafer bow and flattened the (In, Ga)N LEDs-on-Si wafer by designing the buffer layer as AlN/stepgraded-AlN in an MOCVD growth process [65]. Another option can be growing low-dimensional nanostructures [4], including QDs [35] and nanowires [4]. Lateral nanowire strain relief can result in dislocation-free active regions, while large surface-to-volume ratios offer larger electrochemical reaction interaction areas than planar epitaxy [3, 4].

Lattice mismatch between binary group-III nitrides, e.g. InN and GaN or AlN and GaN, is another impediment to epitaxial development. It leads to high defect densities in their ternary compounds. These can act as non-radiative recombination centers and greatly reduce minority carrier lifetimes. Moreover, lattice mismatch can cause many structural defects between layered optoelectronic structures. One example of a structural defect is DBR cracking, which is especially relevant to AlGaN-based UV-emitting vertical-cavity surface-emitting lasers (VCSELs) [66, 67].

In addition, the external strain caused by lattice mismatch between different wurtzite epilayers can lead to piezoelectric polarization and further increases the total polarization fields $[15,16]$. These large polarization fields can reduce electronhole wavefunction overlap and further lead to severe issues, including the quantum confined Stark effect (QCSE), potentially Auger recombination-induced efficiency droop, and carrier leakage $[4,68,69]$. In this case, the active region should be designed carefully to suppress such polarization and achieve better carrier confinement.

1.1.2.2. P-type doping issues. Effective p-type doping is another major issue for group-III nitride optoelectronics. The problem was solved by the Nobel laureates Amano, Akasaki, Nakamura et al when they achieved Mg-doping of GaN during MOCVD growth using an ex situ low-energy electron beam treatment and post-growth annealing [30, 70], and later fabricated the first blue GaN laser [71]. The research field of InGaN/GaN-based LEDs and lasers has since gained a significant boost. However, effective $\mathrm{Mg}$ doping remains somewhat unresolved, mainly because of limited acceptor solid solubility and the high activation energy of $\mathrm{Mg}$ in the GaN matrix, as well as self-compensation of the intrinsic n-type characteristics of native nitrogen vacancies in group-III nitride lattices [72-74]. A high $\mathrm{Mg}$ doping level in the order of $>10^{19}-10^{20} \mathrm{~cm}^{-3}$ is required to yield a carrier concentration in the order of $10^{17} \mathrm{~cm}^{-3}$. However, excessive dopant is known 
to deteriorate crystal quality and cause high optical losses of $\sim 65 \mathrm{~cm}^{-1}$ [2].

Although ultraviolet-C (200-280 nm) AlGaN/GaN-based LEDs have been demonstrated, e.g. $275 \mathrm{~nm}$ LED with EQE of more than $20 \%$ as demonstrated by Takano et al [75], electrically injected ultraviolet-C laser diodes did not exist for a long time. This is because it is even more challenging to activate $\mathrm{Mg}$ in $\mathrm{AlGaN}$ materials compared to that in $\mathrm{GaN}$, as $\mathrm{Al}$ mole fractions increase, as the acceptor activation energy increases from $0.17 \mathrm{eV}$ for $\mathrm{GaN}$ to $0.51 \mathrm{eV}$ for AlN [74]. It was not until 2019 that Zhang et al [76] demonstrated the first AlGaN-based ultraviolet-C laser diode at $271.8 \mathrm{~nm}$ under pulsed current injection. This successful demonstration was attributed to the ability of distributed polarization-induced carrier density enhancement in the p-cladding layer to achieve high conductivity and hole injection with low internal loss. During the global pandemic in 2020, ultraviolet-C light-emitting devices appeared to have a beneficial impact on healthcare governance and the healthcare industry, as they enabled speedy disinfection of unoccupied rooms and public transport vehicles.

1.1.2.3. TE-TM polarization issues. In-plane strain in QWs can affect laser emission due to its strong polarization dependence. In particular, the progressive change from in-plane compressive strain to in-plane tensile strain causes the valence band structures to either be in a degenerate state or split into three valence sub-bands due to crystal field and spin-orbit splitting. In [77, 78], as the $\mathrm{Al}$ increases from 0 to 0.25 mole fraction (see figures 4(a) and (b), respectively), the valence band structure changes from having a heavy hole $\left(\mathrm{HH}, \boldsymbol{\Gamma}_{\mathbf{9 v b m}}\right)$ at the top-most valence sub-band to exhibiting a degenerate state where the valence sub-bands merge. For the mole fraction of 1 , the crystal field split-off hole band $\left(\mathrm{CH}, \boldsymbol{\Gamma}_{\mathbf{7 v b m}}\right)$ crosses over the heavy hole band and became the top-most valence band in AIN (see figure 4(c)). An early description of the valence band structure can be found in [79], and a recent discussion of the related topics of optical polarization and the light-extraction efficiencies of ultraviolet-C AlGaNbased LEDs that emit between $264 \mathrm{~nm}$ and $220 \mathrm{~nm}$ is reported in [80].

Since the electron-HH transition leads to transverse electric (TE)-dominant emission $(\mathbf{E} \perp \mathbf{c})$, while the electron- $\mathrm{CH}$ transition favors the transverse magnetic (TM)-dominant emission $(\mathbf{E} \| \mathbf{c})$, switching from TE- to TM-polarized light occurs as the $\mathrm{Al}$ content increases. The degree of polarization $\left(\mathrm{P}_{\mathrm{TE} / \mathrm{TM}}\right)$ is defined as $\mathbf{P}_{\mathbf{T E} / \mathbf{T M}}=\left(I_{\perp}-I_{\|}\right) /\left(I_{\perp}+I_{\|}\right)$, where $I_{\perp}$ and $I_{\|}$are the integrated photoluminescence intensities for the polarization components of $\mathbf{E}$ perpendicular and parallel to c, respectively. Using $\mathrm{Al}_{x} \mathrm{Ga}_{1-x} \mathrm{~N}$ grown on the sapphire substrate as an example, the corresponding degree of polarization changes from positive in GaN to negative in AlN, as depicted in figure 4 . The broader beam profile of the TM-polarized light results in mode leakage into the p-cladding region and thus reduces AlGaN device performance [77, 78, 85]. Readers are referred to $[81-84,86]$ for further reading on the unique challenges that affect AlGaN development in the ultraviolet-C regime and the optical properties of $\mathrm{GaN}$.

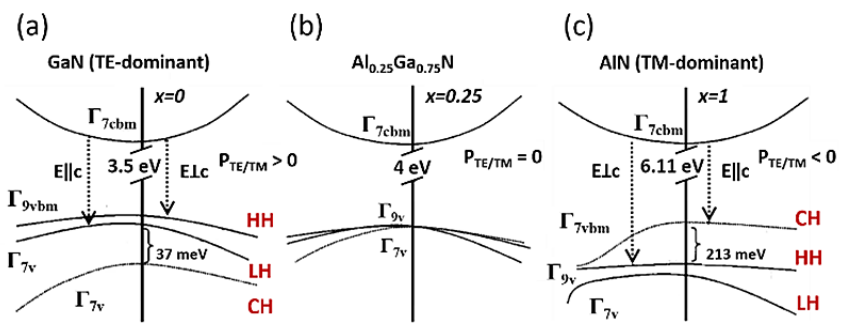

Figure 4. Band structure near the $\Gamma$-valley. The horizontal axis and vertical axis are wavevector and energy respectively. The electron (cbm), heavy hole $(\mathrm{HH})$, light hole ( $\mathrm{LH})$, and crystal field split-off hole $(\mathrm{CH})$ bands for $\mathrm{Al}_{\mathrm{x}} \mathrm{Ga}_{1-\mathrm{x}} \mathrm{N}$ materials grown on sapphire with Al mole fractions of: (a) $\mathrm{x}=0(\mathrm{GaN})$, TE-(polarized light)-dominant, (b) $\mathrm{x}=0.25$, (c) $\mathrm{x}=1$ (AlN), and TM-(polarized light)-dominant are shown. The symbols $\mathbf{P}_{\mathbf{T E} / \mathbf{T M}}, \mathbf{E}, \mathbf{c}, \mathrm{cbm}$, and vbm denote the degree of TE/TM polarization, electric field, [0001] crystal orientation, conduction band minimum, and valence band maximum, respectively. The dotted arrows are offset from the $\Gamma$-valley for clarity. Adapted from [77], with the permission of AIP Publishing. Prior investigation of the valence band structure is provided in [79] and a recent discussion of device implementation for ultraviolet-C AlGaN-based LEDs between $264 \mathrm{~nm}$ and $220 \mathrm{~nm}$ is reported in [80]. The band structure of $\mathrm{AlGaN}$ and related properties are widely documented by multiple sources [81-84].

1.1.3. State-of-the-art devices and applications. Group-IIInitride optoelectronics are attractive mostly because of their superior material characteristics, such as broad bandgap tunability, thermal and chemical stability, and relatively mature growth techniques. Therefore, despite the challenges mentioned above, excellent optoelectronic devices, e.g. LEDs, lasers, superluminescent diodes and photodetectors, have been demonstrated via growth optimization and meticulous device design.

As mentioned before, active-region polarization fields can lead to QCSE and reduced radiative recombination efficiency. Hence, suppression of undesired polarization fields in the active region is essential to various optoelectronic devices. There are several reports on tailoring group-III element mole fractions in QWs and quantum barriers to enhance laser recombination efficiency [87-89]. Another solution that has received significant attention is the growth of device structures on native non-polar and semipolar GaN substrates [90-93]. This solution seeks to address several polarization-field related problems that affect $c$-plane GaN devices [94], e.g. reduction of carrier recombination lifetimes and increase in the electron-hole wavefunctions overlap, to provide higher radiative recombination efficiencies. The use of native non-polar and semipolar GaN substrates has also been demonstrated to produce higher In incorporation efficiency than a $c$-plane substrate, thus allowing the growth of devices such as green LEDs that require high In contents [95].

However, in AlGaN-based deep-UV devices, the polarization field helps to tackle p-type doping issues as shown in figure 5. Conventional thermally activated doping of the structure in figure 5(a) produces a limited activated hole carrier concentration. As shown in figure 5(b), the polarization field $(\boldsymbol{P})$ in the graded-AlGaN layer results in a bulk bound 
(a)

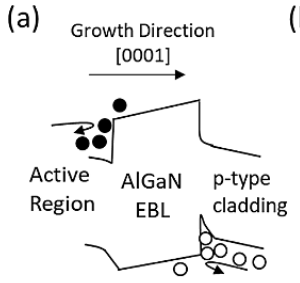

(b) Growth Direction
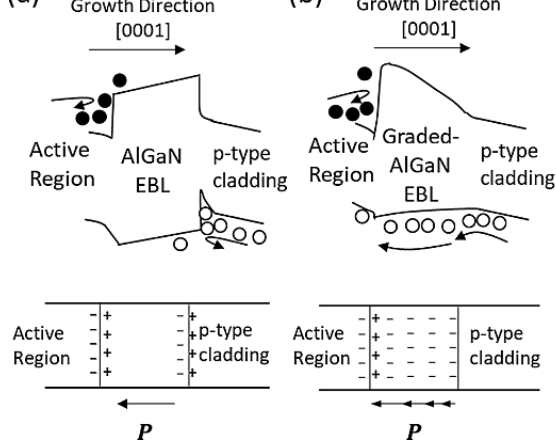

(c) Growth Direction



$\bullet$ electrons

Figure 5. Band diagrams and polarization-induced charge distributions in (a) bulk-AlGaN, (b) graded-AlGaN, and (c) SL-AlGaN EBLs. Representations of interface and bulk charges are included for (a) and (b), respectively. $\boldsymbol{P}$ is the polarization vector.

charge $\rho_{\text {bound }}=-\nabla \cdot \boldsymbol{P}$. These unbalanced negative polarization charges exist as a mobile 3D electron slab/gas and create an electric field. Holes can be field-ionized from the deep acceptor level to compensate for the negative polarization charge, thereby increasing the free hole concentrations. In similar scenarios, the term polarization-induced 'doping' has been widely used in the literature, which should be physically referred to as an increase in 'carrier' concentrations (not the dopant concentration). Further, a graded-AlGaN layer [76, 96-99] can assist the hole transport from the p-type cladding layer towards the active regions [100-102]. As shown in figures 5(a)-(c), hole transport can be facilitated by replacing the bulk AlGaN electron blocking layer (EBL) with a gradedAlGaN EBL or alternating AlN/AlGaN superlattice (SL) layers, thereby reducing the valence band offset or increasing the hole tunneling probability $[96,97,103,104]$.

While blue InGaN-based LEDs and laser diodes have received significant attention and broad adoption for solidstate lighting, the green LED still suffers from a low external quantum efficiency (EQE) of $<60 \%$ because the strain and QCSE increase with the indium mole fraction in the active region and because of the lack of an effective p-doping method $[105,106]$. This is far from the approximately $80 \%$ peak EQE reported for the violet-blue wavelength region using groupIII nitrides [107-109] and for the red wavelength region using group-III arsenide/phosphide materials [110]. Thus, we reference the well-known 'green gap' in the vicinity of $530 \mathrm{~nm}$ [111]. It is noted, however, that the peak EQE of an InGaNbased LED can be as high as $53.3 \%$ in the green wavelength region, as demonstrated by Li et al [112]. A peak EQE of $32.7 \%$ was demonstrated in the yellow wavelength region by Jiang et al [113]. In this work, the 'green-yellow gap' references the range of approximately $530-600 \mathrm{~nm}$.

To improve quantum efficiencies, green InGaN-based LEDs have been optimized via surface roughening of the $\mathrm{p}-\mathrm{GaN}$ layer, as well as the introduction of V-pits in the green LED layer structure. In particular, the V-pits produce epitaxy that suppresses diffusion of charged carriers into the non-radiative recombination centers formed at threading dislocations $[114,115]$. Despite the green LED efficiency

improvements mentioned above and the recent development of Watt-class $532 \mathrm{~nm}$ laser diodes by Nichia, as well as $530 \mathrm{~nm}$ and $465 \mathrm{~nm}$ laser diodes on semipolar substrates by Sony and Toyoda Gosei with peak wall-plug-efficiencies of $\sim 19 \%$, $\sim 18 \%$, and $\sim 37 \%$ respectively [116, 117], efficient true-green $(555 \mathrm{~nm})$ laser diodes have yet to be demonstrated. Devices with emission near the true-green color are essential to matching the peak sensitivity of human photopic vision, and thereby achieving the highest possible luminous intensity and efficacy. These (true-)green-emitting laser devices are needed for spectroscopy, picoprojectors, and micro-display applications [118].

\subsection{Halide-perovskite semiconductors}

The inherent bandgap tunability of halide perovskite resembles that of group-III nitrides. Halide perovskite was first discovered by the German mineralogist Gustav Rose in the Ural Mountains of Russia in 1839. It was then named after the Russian mineralogist, Count Lev Alekseyevich von Perovski [119]. This situation remained until 1893, when Wells reported the synthesis of bulk perovskite compounds from aqueous solutions [120]. The crystal structure of perovskite was first described by Norwegian mineralogist Victor Moritz Goldschmidt in 1926 [121] and subsequently established by Helen Dick Megaw, a renowned Irish x-ray crystallographer, in 1945 [122]. Since then, this material system has received substantial attention that has led to several key reports, such as that of the first hybrid organic-inorganic perovskite by Weber in 1978 [123, 124], as well as the development of perovskitebased solar cells following their first demonstration by Miyasaka et al in 2009 [125].

1.2.1. Material properties. Perovskite material has the chemical form $\mathrm{ABX}_{3}$ and the same crystallographic structure as $\mathrm{CaTiO}_{3}$, as shown in figure 6. The perovskite crystal structure is an ideal cubic unit cell with cation $\mathrm{A}$ in the center with 12-fold cuboctahedral coordination, cation B at the corners with six-fold coordination, and anion $\mathrm{X}$ at the midpoints of the edges. However, it is noteworthy that the crystal structure can transform between cubic, tetragonal, and orthorhombic phases depending on the ionic or elemental radii of the elements [126, 127].

While oxide perovskites, in which $\mathrm{X}$ is an oxygen anion, exhibit interesting features such as piezoelectricity, ferromagnetism, magnetoresistance, and multiferroic properties [129], halide perovskites, where $\mathrm{X}$ is a halide anion, are widely used in optoelectronic devices due to their inherent semiconducting properties. The latter can be further categorized into inorganic and hybrid organic-inorganic halide perovskites. Hybrid organic-inorganic halide perovskites are made by substituting an organic cation for A. The most common types of halide perovskites are shown in table 2. Furthermore, readers are referred to a review article by Ono et al [130] that addresses progress in organic-inorganic halide-perovskite materials and devices.

As with various group-III-nitride (e.g. GaN, InN, and AlN) and colloidal group-II-VI (e.g. CdSe) materials, perovskites 


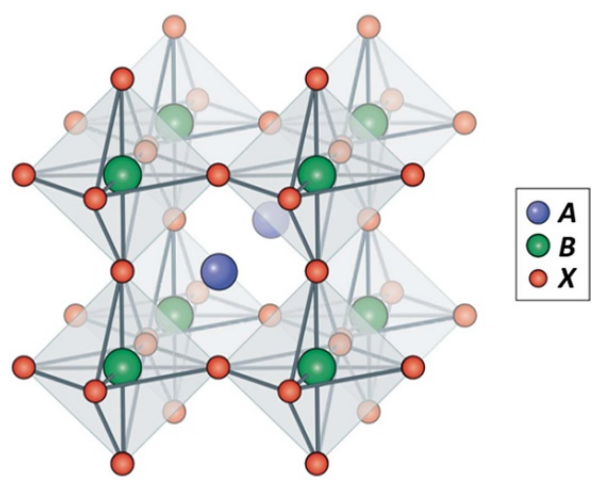

Figure 6. Perovskite crystal structure of $\mathrm{CaTiO}_{3}$ with the general formula of $\mathrm{ABX}_{3}$. Adapted by permission from Springer Nature Customer Service Centre GmbH: Springer Nature, Nature Reviews Materials [128], (C) 2019.

Table 2. Common types of halide perovskites.

\begin{tabular}{ll}
\hline Inorganic perovskite & Organic-inorganic perovskite \\
\hline $\mathrm{CsPbBr}_{3}$ & $\mathrm{MAPbI}_{3}{ }^{\mathrm{a}}$ \\
$\mathrm{CsPbCl}_{3}$ & $\mathrm{MAPbBr}_{3}{ }^{\mathrm{a}}$ \\
$\mathrm{CsPbI}_{3}$ & $\mathrm{MAPbI}_{3-x} \mathrm{Cl}_{x}{ }^{\mathrm{a}}$ \\
- & $\mathrm{FAPb}_{\left.\mathrm{I}_{1-x} \mathrm{Br}_{x}\right)_{3}{ }^{\mathrm{b}}}$ \\
- & $\mathrm{FAPbBr}_{3}{ }^{\mathrm{b}}$ \\
\hline
\end{tabular}

a MA refers to methylammonium $\left(\mathrm{CH}_{3} \mathrm{NH}_{3}\right)$.

${ }^{b}$ FA refers to formamidinium $\left(\mathrm{CH}\left(\mathrm{NH}_{2}\right)_{2}\right)$.

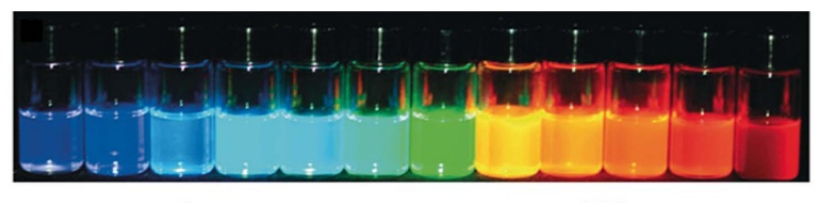

Cs

MA

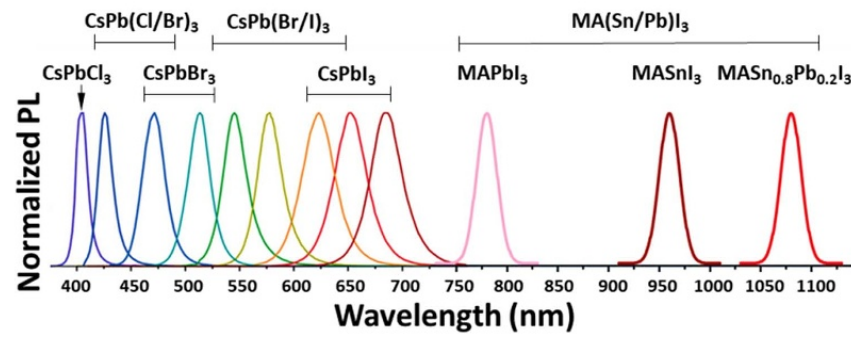

Figure 7. Normalized photoluminescence (PL) spectra of compositionally tunable metal-halide-perovskite semiconductors and their wavelength-tunability across the visible-to-NIR regime. [131] John Wiley \& Sons. Copyright (c) 2018 WILEY-VCH Verlag $\mathrm{GmbH} \& \mathrm{Co}$. KGaA, Weinheim.

exhibit typical semiconductor direct bandgaps. The metalhalide perovskite has a general formula of $\mathrm{ABX}_{3}(\mathrm{X}=\mathrm{Cl}$, $\mathrm{Br}$, or I). Depending on the constituent elements and composition, this class of semiconductor exhibits wide wavelength tunability across the visible-to-NIR region, i.e. from $400 \mathrm{~nm}$ to $1.1 \mu \mathrm{m}$ (see figure 7).

The tunable emission wavelength characteristics of halide perovskite within the visible light spectrum can be explored using various strategies. One of the most explored methods


2D perovskite
nanosheet
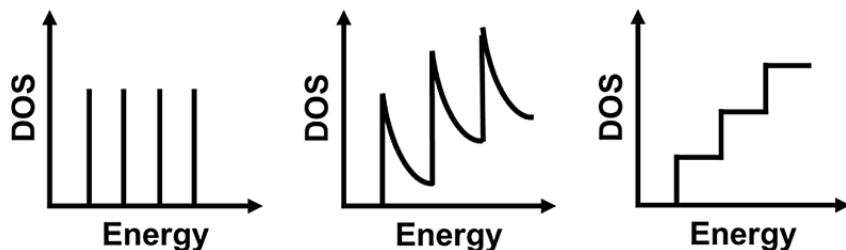

Figure 8. Schematic illustrations and DOSs versus energy for halide perovskites with various dimensionalities. Reproduced from [142] with permission of The Royal Society of Chemistry.

is the exploitation of quantum-size effects. It is well known that nanoparticle dimensionality changes lead to emission wavelength changes [132]. However, the most direct way to achieve tunability is via anion or cation substitution. For instance, Cs cation substitution for $\mathrm{MA}$ in a $\mathrm{CsPbBr}_{3}$ nanoparticle can change its emission wavelength from $\sim 510 \mathrm{~nm}$ to $\sim 525 \mathrm{~nm}$ [133].

Similarly, exchanging the anion of $\mathrm{CsPbX}_{3}$ changes the corresponding bandgap from $3.11 \mathrm{eV}\left(\mathrm{X}=\mathrm{Cl}^{-}\right)$to $2.3 \mathrm{eV}$ $\left(\mathrm{X}=\mathrm{Br}^{-}\right)$or $1.55 \mathrm{eV}\left(\mathrm{X}=\mathrm{I}^{-}\right)[133,134]$. Easy solutionprocessable emission wavelength tuning is highly advantageous for various optoelectronic devices. For laser-based devices, tunable emission from perovskite-based materials has also been reported simply by varying the nanoimprinted perovskite thin film grating period $[135,136]$. Thus, the possibility of covering the entire visible spectra using perovskitebased materials is highly attractive and worth exploring with regards to optoelectronic device gain media.

Furthermore, low-dimensional nanostructures of metalhalide perovskites based on nanoplatelets, nanowires, and QDs have received significant attention because of their inherent quantum size effects. It has been reported that these low-dimensional nanostructures exhibit enhanced photogenerated carriers because they offer higher crystallinities, larger quantum confinement effects, and larger surface-to-volume ratios than their bulk counterparts [137-139]. For lightemitting and lasing devices, both one-dimensional (1D) and zero-dimensional (OD) nanostructures have received extensive attention because of their high photoluminescence quantum yields (PLQYs), which are driven by large quantum confinement effects, as well as because of their high crystallinities and low defect state contents [140, 141]. Figure 8 shows the effect of dimensionalities of halide perovskites on their corresponding densities of states (DOSs), which resemble those of group-III-nitride semiconductors.

Broad operating wavelengths are ideal for various optoelectronic devices such as solar cells [131, 143-145], radiation detectors [146], photodetectors [147-150], LEDs [151, 152], and lasers [128, 153]. Solution-based processing is typically 
utilized to avoid the need for costly, rigorous epitaxial growth technologies, such as MBE and MOCVD, when producing low-cost optoelectronic devices. As mentioned above, it remains challenging to achieve high wall-plug efficiencies among group-III-nitride light-emitting devices that operate in the 'green-yellow gap'. Hence, halide-perovskite could fill in the wavelength gap where group-III nitrides is inefficient.

1.2.2. Material growth and key issues. Compared to groupIII-nitride semiconductors, which typically rely on epitaxial growth methods such as MBE, MOCVD, and PLD, perovskite-based materials can be synthesized via solutionphase growth or CVD [137, 139, 142]. In particular, lowdimensional perovskite nanostructures, e.g. QDs or nanowires, can be prepared primarily via solution-phase growth.

While solution-phase growth is relatively low-cost and helps researchers to understand fundamental material properties, CVD-based growth of perovskite-based materials has received increasing attention in recent years, as it offers better scalability than solution-based growth methods [137]. Vaporphase growth has been used to produce perovskite-based multiple quantum well (MQW) structures [154] similar to those produced via epitaxial growth of group-III-nitride semiconductors. Such methods offer higher flexibility and scalability so that we can provide sub-micrometer thicknesses and layered structures, as well as crystallinity favorable to the design of perovskite-based optoelectronic devices.

While the synthesis method of perovskite has reached a certain level of maturity, the pressing photostability issue related to perovskite-based material and devices, especially under exposure to high temperature and moisture conditions, has also improved significantly in recent years. During the early development, a lack of stability constrained the practical application of perovskite-based materials, particularly for photovoltaic (PV) applications, where stable operation is required for reliable characterization and atmospheric environment device applications. Air-stable operation has become one of the key requirements for sustained development in this field. To move towards commercialization, many groups have sought to improve and prolong the operational stabilities of perovskite-based devices using either intrinsic (via e.g. mixing with different cations, stoichiometric changes, surface passivation, or core/shell structures) [155-157] or extrinsic modifications (e.g. coating with an encapsulation layer) [158, 159]. Readers are referred to the comprehensive review article by Castro-Hermosa et al [160] that addresses stability in perovskite devices.

The Goldschmidt tolerance factor $(t)$ in equation (1) is often used as a rule of thumb when improving material stability:

$$
t=\frac{r_{\mathrm{A}}+r_{\mathrm{X}}}{\sqrt{2}\left(r_{\mathrm{B}}+r_{\mathrm{X}}\right)}
$$

where $r_{\mathrm{A}}, r_{\mathrm{B}}$, and $r_{\mathrm{X}}$ are the effective radii of the ions in the perovskite $\mathrm{ABX}_{3}$ structure [161]. Importantly, the size of the A-cation may allow it to induce distortion in perovskite crystal phases [162]. A typical tolerance factor of between 0.8 and 1 is known to maintain the formation of the perovskite structure. For cases in which the A-cation is too small (i.e. $t<0.8$ ) or too large (i.e. $t>1$ ), it will distort the formation of perovskite, and may result in alternative non-perovskite structures [163]. Inorganic cations such as $\mathrm{Cs}^{+}$typically yield better thermal, moisture, and optical stabilities than organic-based cations such as MA (methylammonium, $\mathrm{CH}_{3} \mathrm{NH}_{3}$ ) and $\mathrm{FA}$ (formamidinium, $\mathrm{CH}\left(\mathrm{NH}_{2}\right)_{2}$ ). For instance, the all-inorganic $\mathrm{CsPbBr}_{3}$ perovskite is widely used in LEDs [164-166] and optically pumped laser devices [136, 167, 168].

1.2.3. State-of-the-art devices. Metal-halide perovskites, which have outstanding optoelectronic properties, were first demonstrated as PV cells in 2009 by Miyasaka et al [125]. Since then, the perovskite-based solar cell research community has grown substantially. Over the years, the power conversion efficiency (PCE) has increased from 3.8\% at the first demonstration to $25.2 \%$, as demonstrated in 2019 by a team from the Korea Research Institute of Chemical Technology and Massachusetts Institute of Technology [169]. Oxford Photovoltaics has achieved a PCE of $28 \%$ using perovskitesilicon tandem solar cells [170], surpassing various other categories of solar cells, such as single-junction $\mathrm{Si}$, cadmium telluride, CuInGaSe (CIGS), and a-Si:H solar cells. Such achievements within this short timeframe may suggest that perovskite-based materials offer immense potential for various other optoelectronic devices because of their easy processability and the compositional tunability of halide-perovskite materials.

In fact, halide-perovskite materials have already received significant attention for applications related to LEDs and photodetectors. For instance, green-emitting $\mathrm{Br}_{3}$-based LEDs with EQEs of up to $17.5 \%$ and $20.3 \%$ have been demonstrated by Zhang et al [171] and Lin et al [172], respectively. While these EQEs are far from those offered by InGaN-based LEDs, easy solution-processability makes these materials attractive for applications in solid-state lighting and nextgeneration display technologies [164, 173-177]. In addition, various perovskite-based photodetectors with high responsivities [178-180], flexibility [181-184], and fast response speeds [150, 178, 185-187] have also been demonstrated widely [188].

The extraordinary properties of lead-halide perovskite have enabled the aforementioned optoelectronic applications. This is because of their favorable emission linewidths and the ability to tune direct bandgaps by simply modifying chemical compositions [133, 189]. Using quantum-confined nanostructures, perovskite also exhibits stimulated emission with low lasing thresholds due to high defect tolerance, high optical gain, long carrier lifetime, long charge carrier diffusion lengths, and high optical absorption coefficients [153]. In particular, the inherent advantage of high optical gain $\left(>3000 \mathrm{~cm}^{-1}\right.$ ) [190] exhibited by perovskite thin films is attractive for applications in micro- and nano-lasers. This creates an alternative research path that is distinct from the technologically mature group-III-nitride platform. 




Figure 9. A complete suite of halide-perovskite-based optoelectronic devices, including solar cells, LEDs, and photodetectors, that have received substantial attention. Further device exploration includes the potentially viable light emitters based on electrically driven laser and ASE devices to complement group-III-nitride-based materials in various photonic applications.

This motivates the present review, which discusses the technological readiness of both classes of semiconductors with regard to light-emitting applications. We consider both horizontal waveguide devices (Fabry-Pérot lasers and superluminescent diodes, as well as related amplified spontaneous emission (ASE) devices) and vertical waveguide devices (VCSELs or ASE devices), respectively, in section 2 (group-III nitrides) and section 3 (halide perovskites). In section 3 , we further discuss the heterogeneous integration of halide perovskites and group-III nitrides into an integrated gain medium for laser or ASE devices, and thus explain the relevance of both classes of semiconductors.

Figure 9 summarizes key perovskite-based devices that are currently receiving focused attention (e.g. solar cells, LEDs, and photodetectors), as well as the desired perovskitebased components, such as electrically driven lasers and ASE devices. Significant effort is still required to accelerate progress in using halide-perovskite semiconductors to produce a complete suite of electrically injected perovskite-based optoelectronic devices.

The use of halide-perovskite is still in its infancy as compared to the group-III-nitride light-emitters, which is likely to remain the mainstay for laser technology. This review set off to steer the interested research community and readers toward utilization of halide-perovskite for eventual electrically injected tunable wavelength lasing and ASE applications. Section 3 brings out the recent limited hybrid group-III-nitride/halideperovskite examples (section 3.1) before reviewing the relevant halide-perovskite lasing and ASE devices (section 3.2). Although the approach of combining the robust process technology of group-III nitride with the ease of processing and tunable wavelength (by composition tuning) of the halideperovskite is challenging, the future realization of electrically injected halide-perovskite can potentially be resolved by the group-III-nitride/halide-perovskite hybrid. It is believed that the topic is a new research direction that could harness the effort of the research community at large.

\section{Group-III-nitride lasers and superluminescent diodes}

In this section, we first review the techniques of Fabry-Pérot lasers and their integrated variants, such as single-section and multi-section laser diodes (section 2.1). Next, we highlight the unique properties of distributed feedback (DFB) laser, which incorporates a surface grating for achieving narrow-linewidth emission (section 2.2). Finally, the technology of superluminescent diodes is described in section 2.3.

\subsection{Fabry-Pérot and integrated lasers}

2.1.1. Overview. Fabry-Pérot cavities are the mainstay of semiconductor lasers as a light source, which is the focus of this section. However, to shed light on new integrated optical functionalities of a modulator and a photodetector, additional sub-sections are included. Prior to the work described herein, a different approach to enhance the laser functionality was undertaken by Chua et al by introducing additional contacts resulting in a laser that can serve as an optoelectronic 'AND' gate [191], a photo triggerable laser [192] and a beam sweeping laser [193]. Central to all these concepts is the manipulation of the gain medium with differential current pumping either through multiple contacts in one cavity or in separate cavities.

Fabry-Pérot laser diodes based on the group-III-nitride system are commonly designed to operate at $405 \mathrm{~nm}$ (violet), $450 \mathrm{~nm}$ (blue), and $520 \mathrm{~nm}$ (green). These devices are essential to consumer electronics and optoelectronics and are already widely reported as discrete devices for applications in optical communication [194, 195], biology [196-198], and cuttingedge instrumentation [199, 200]. However, the group-IIInitride laser diode family requires a substantial market push for the demonstration of photonic integrated circuits and integrated device architectures, which are well established for their near-infrared counterparts [201, 202]. The desired technologies include monolithic tunable lasers, integrated mode-locked lasers, and multisection laser-modulator-amplifier devices.

Conventionally, a laser diode [71] is composed of a layered epitaxial structure with a horizontal waveguide design comprising an active region, a separate confinement heterostructure, cladding, and contact layers. Subsequent fabrication of ridge-waveguides enhances lateral mode confinement via index or gain guiding. Further formation of the laser cavity is achieved via substrate thinning and facet cleaving or etching to form parallel mirrors, which are further coated to increase the modal gain and improve the optical properties. Metal contact deposition completes the fabrication, forming an electrically injected Fabry-Pérot laser diode (see figure 10(a)).

A general form of a multi-section InGaN-based laser diode involves isolating the contact region of the laser structure to obtain separate sections (e.g. the gain, absorber, modulator, and photodetector). The resulting sections are therefore electrically isolated but coupled because the cladding, waveguide, and active region remain connected. For example, a two-section laser diode (see figure 10(b)) can be implemented for purposes such as generation of high-power ultrashort 


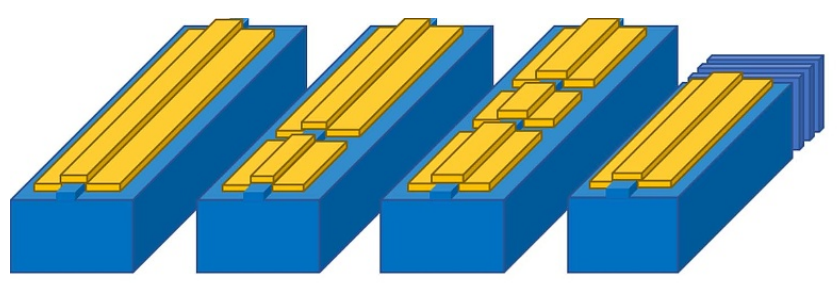

(a)

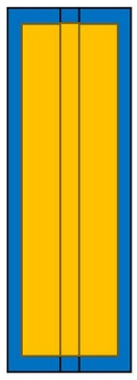

(b)

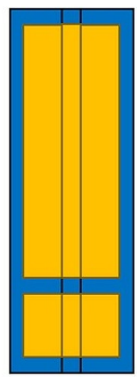

(c)

(d)
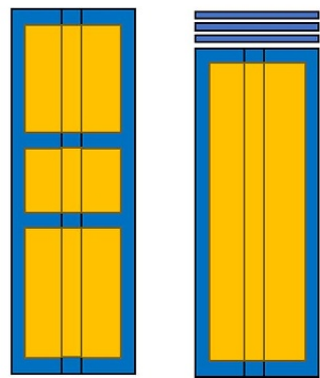

Figure 10. Elevation and top views of: (a) a conventional ridge-waveguide laser diode, (b) a two-section laser diode, (c) a three-section laser diode, and (d) a laser diode with an integrated optical element (e.g. an air/semiconductor DBR).

pulses [203], superluminescence [204], superradiance [205], light amplification [206], Q-switching [207], mode-locking [208], or fast modulation [209]. Variants of the multi-section laser, such as three-section devices, have been developed using the same principle (see figure 10(c)). Concepts such as gain-absorber-gain [210], and gain-modulator-photodetector [211] integrated devices open the path for highly tunable selfpulsating lasers and photonic integrated circuits. Other types of multi-section lasers include tapered structures for laser amplification [212] and diffraction gratings for vertical emission [213], which can incorporate external elements such as DBRs [214] (see figure 10(d)).

It is noted that the reported work on multi-section lasers focused on wavelengths between $400 \mathrm{~nm}$ and $450 \mathrm{~nm}$. Lasing emission at longer wavelengths using group-III-nitride multisection lasers clearly represents a gap to be addressed.

\subsubsection{Device performance and applications}

2.1.2.1. Integrated laser-modulator. One of the main advantages of integrated modulators is their small footprint, which leads to low capacitance and high-speed modulation. In these devices, the principle of operation is the electroabsorption modulation, i.e. the control of the optical absorption in the modulator section by applying an electric field. For GaN-based devices, the piezoelectric fields found in the InGaN QWs enable this scheme even though the laser and the modulator section share the same active region. By applying a reverse voltage at the modulator section, the piezoelectric fields of InGaN are compensated and its absorption edge is modified, counteracting the QCSE and changing the effective bandgap of the modulator section. Utilizing these characteristics, it is possible to turn the laser on and off by simply modifying the

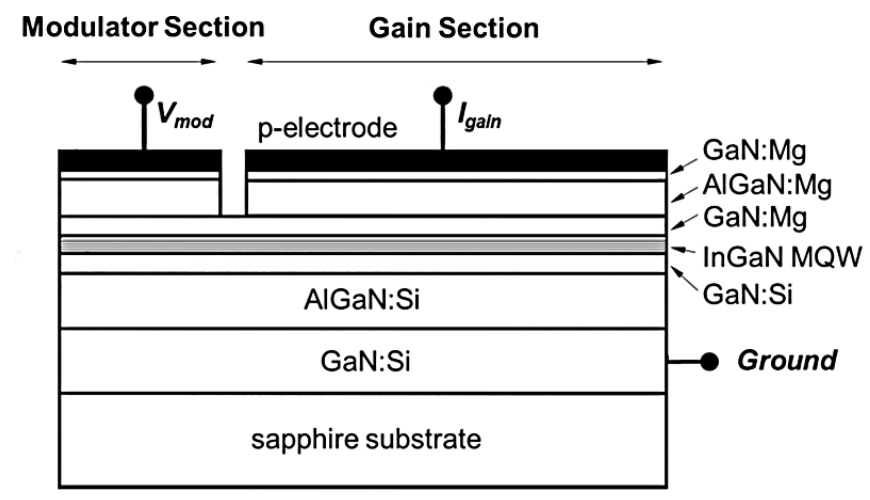

Figure 11. Schematic of a modulator-integrated two-section GaN laser diode showing the $100 \mu \mathrm{m}$ modulator section (modulation voltage, $V_{\text {mod }}$ ), $700 \mu \mathrm{m}$ gain section (operating current, $I_{\text {gain }}$ ), and a common ground. The depicted laser diode includes the following layers grown on a sapphire substrate: p-electrode, top $\mathrm{GaN}: \mathrm{Mg}$ and bottom GaN:Si contact layers, core structure consisting of $\mathrm{GaN}: \mathrm{Mg}$ and GaN:Si waveguide layers that encapsulate MQW charge-carrier confinement layers, and a doped $\mathrm{AlGaN}$ optical-confinement waveguide structure. Adapted from [215], with the permission of AIP Publishing.

voltage at the modulator section. This is promising for highspeed applications such as visible light communication and high-frequency laser displays.

Two-section InGaN laser diodes with integrated modulators were first reported by Kneissl et al in $2002(\lambda=401 \mathrm{~nm}$, where $\lambda$ is the operating wavelength), as shown in figure 11 [215]. A $15 \mu \mathrm{m}$ shallow-trench isolation was used to create a front-facet modulator section $(100 \mu \mathrm{m})$ that operated under reverse bias down to $-7.2 \mathrm{~V}$ with absorption-change as large as $5000 \mathrm{~cm}^{-1}$ and power modulation from approximately $3 \mathrm{~mW}$ to $0.5 \mathrm{~mW}$. These parameters resulted in an on/off ratio $\left(\mathrm{R}_{\mathrm{on} / \mathrm{off}}\right)$ of $\sim 7.8 \mathrm{~dB}$ and a modulation efficiency $\left(\mathrm{R}_{\mathrm{on} / \mathrm{off}} / \Delta \mathrm{V}\right)$ of $\sim 1.1 \mathrm{~dB} \mathrm{~V}^{-1}$. The device had a $3 \mu \mathrm{m}$ wide ridge-waveguide and a $700 \mu \mathrm{m}$ long gain section. Due to the piezoelectric builtin fields in $c$-plane GaN, the modulator exhibited the lowest extinction at the highest reverse bias. Further progress was made more than 10 years later.

Shen et al reported an integrated $2 \mu \mathrm{m}$ wide ridgewaveguide laser-modulator in a semipolar $(20 \overline{21}) \mathrm{GaN}$-based platform $(\lambda=448 \mathrm{~nm})$ [209]. A $200 \mu$ m modulator section was isolated from a $1290 \mu \mathrm{m}$ laser gain section by a $10 \mu \mathrm{m}$ shallow trench. Unlike the modulator from $c$-plane $\mathrm{GaN}$, this semipolar device exhibited a higher extinction at $-3.5 \mathrm{~V}$, with power modulation from $15.9 \mathrm{~mW}$ to $1.8 \mathrm{~mW}$ leading to an on/off ratio $\left(\mathrm{R}_{\mathrm{on} / \mathrm{off}}\right)$ of $9.4 \mathrm{~dB}$ and a modulation efficiency $\left(\mathrm{R}_{\mathrm{on} / \mathrm{off}} / \Delta \mathrm{V}\right)$ of $2.68 \mathrm{~dB} \mathrm{~V}^{-1}$. A larger modulation efficiency of $4.5 \mathrm{~dB} \mathrm{~V}^{-1}$ was also reported using a similar structure $(\lambda=404 \mathrm{~nm})$ based on semipolar $(20 \overline{21}) \mathrm{GaN}$ [216]. The improved modulation efficiency of the semipolar GaN platform is characteristic of the increased wavefunction overlap [217], alongside reduced built-in piezoelectric fields and the corresponding band edge dynamics [218].

Feng et al reported on an integrated laser-modulator $(\lambda=412.8 \mathrm{~nm})$ with a third photodetector section on a silicon substrate [211]. A $4 \mu \mathrm{m}$ wide, $790 \mu \mathrm{m}$ long ridge-waveguide 
gain section preceded a $190 \mu \mathrm{m}$ long modulator and a $290 \mu \mathrm{m}$ photodetector, all of which were electrically isolated by a $10 \mu \mathrm{m}$ wide shallow trench. Power modulation (as measured at the photodetector section) from approximately $30 \mathrm{~mW}$ to $22 \mathrm{~mW}$ corresponded to modulator bias from $2 \mathrm{~V}$ to $-4 \mathrm{~V}$, thus giving an $\mathrm{R}_{\mathrm{on} / \mathrm{off}}$ of $1.35 \mathrm{~dB}$ and modulation efficiency of $0.22 \mathrm{~dB} \mathrm{~V}^{-1}$. Further improvement of $\mathrm{GaN}$-on-Si platform may enable large-scale, low-cost integration while retaining the high modulation efficiencies of lasers grown on native substrates.

In terms of the applications of the integrated lasermodulator based on the GaN-based platform, Shen et al demonstrated optical communications of up to $1.7 \mathrm{Gbit} \mathrm{s}^{-1}$ [216] with a modulation bandwidth of $\sim 1 \mathrm{GHz}[209,216]$, limited by the bandwidth of the characterization setup, i.e. the receiver. Nevertheless, these demonstrations are not conclusive regarding the modulation bandwidth of integrated GaN laser-modulators. No other reports have been made on this platform, and thus the full potential of GaN-based integrated laser-modulator has yet to be realized. As a reference, a traditional group-III-V-based integrated lasermodulator is capable of achieving $\sim 14 \mathrm{GHz}$ of modulation bandwidth and $10 \mathrm{Gbit} \mathrm{s}^{-1}$ transmission speed [219]; however, unlike the GaN platform, the group-III-V devices require selective area growth, and thus their realization is more involved.

\subsubsection{Integrated laser-saturable-absorber. The ability} to control the absorber section independently and at different laser cavity locations (see figure 12) enables broad tunability across different regimes of operation. Among the various functions that have been developed based on the laser-saturable-absorber structure, we should highlight the self-pulsating laser. The role of the absorber section in generating a pulsed laser beam has been a subject of detailed investigation that includes observation of self-pulsating operation at zero absorber bias [220]. Figure 13 shows ps-scale pulses from a three-section laser with a saturable absorber integrated at its center. Self-pulsating lasers offer high peak output power in a compact form and have the potential to replace conventional solid-state lasers used in multiphoton physics and other applications.

Initially reported by Tronciu et al in 2004 [222] $(\lambda=400 \mathrm{~nm})$, self-pulsation frequencies between $1.6 \mathrm{GHz}$ and $2.2 \mathrm{GHz}$ were achieved using a so-called 'tandem' laser diode, where a $52.3 \mu \mathrm{m}$ long absorber section was grounded while biasing a $490 \mu \mathrm{m}$ long gain section. Since then, Q-switching and mode-locking of multi-section laser diodes with integrated saturable absorbers have been the subjects of numerous reports $(\lambda=400 \mathrm{~nm}$ to $\sim 430 \mathrm{~nm})$ [203, 207, 208, $210,220-240]$ that cover a range of self-pulsating frequencies and pulse widths as small as 2 ps. Moreover, femtosecond pulse durations with peak power and energy levels of up to $9 \mathrm{~kW}$ and $3 \mathrm{~nJ}$, respectively, have been reported by integrating these multi-section lasers into an external cavity configuration, e.g. to form the GaN-based master oscillator power amplifier (MOPA) [236].



Figure 12. Integrated laser-saturable-absorbers with three-section Gain (G)-Absorber (A)-Gain (G) (left) and two-section Gain (G)-Absorber (A) (right) configurations. Adapted with permission from [221]. Copyright (2013) Society of Photo-Optical Instrumentation Engineers (SPIE).



Figure 13. Pulsing characteristics of an integrated laser-saturable-absorber based on a three-section Gain (G)-Absorber (A)-Gain (G) configuration, as seen via a streak camera. The saturable absorber is maintained at zero bias. Adapted from [220]. (C) IOP Publishing Ltd. All rights reserved.

Q-switching refers to changing the quality factor (Q-factor) of the laser cavity via the absorber section. A low Q-factor is present when light generated in the gain section is captured by the absorber section (usually under a high reverse bias) [231]. Upon increasing electrical injection into the gain section, the absorber continues to capture light until it achieves optical transparency. This suddenly allows the laser cavity to achieve a high Q-factor. This process creates a sudden release of laser light in the form of a pulse. This rapid release of energy depletes the excited carriers in the gain section and the absorber becomes opaque again, and is ready to repeat the Q-switching process once again. In a mode-locked laser, self-pulsation involves synchronous phase relations among the various longitudinal cavity modes of the laser. The absorber section can be selectively modulated or reverse biased to influence the phases of the various longitudinal modes [229], thus creating losses for out-of-phase modes. This means that pulse formation in a mode-locked laser simply occurs when the various longitudinal modes arrive at the output facet simultaneously. Therefore, the pulsation frequency of a modelocked laser is related to the roundtrip time inside the cavity. Consequently, Q-switching typically provides higher optical power levels than mode-locking, but mode-locked lasers may achieve higher repetition rates. It is expected that exploring these types of self-pulsating lasers across the visible spectrum may enable new imaging capabilities, such as compact 
two-photon microscopy and on-chip multi-photon applications. The reader can refer to detailed descriptions of both Qswitched and mode-locked lasers such as those in [241] and [242].

Monolithic integration of the saturable absorber in a laser diode also enables investigation of the concept of ultrashort pulse generation created by superradiance and superfluorescence using InGaN-based emission across wavelengths in the violet-blue color range (i.e. $\lambda=408 \mathrm{~nm}$ to $\sim 423 \mathrm{~nm}$ ) [205, 243-247]. Nonetheless, achieving superradiance and superfluorescence in semiconductors appears to be a controversial topic that requires further examination.

In a different role, the integrated absorber section can be used to suppress lasing. This leads to the production of a superluminescent diode that operates in the ASE regime [204, 234, 248-251]. In section 2.3, the InGaN-based superluminescent diode is discussed separately, and a review article is recommended for further reading.

\subsubsection{Semiconductor optical amplifier integrated laser. A} two-section laser diode that implements a semiconductor optical amplifier (SOA) section is attractive for optical amplification of modulated or continuous wave (CW) laser signals. Under electrical injection, an SOA produces excited carriers leading to population inversion. This is needed to amplify incoming light via stimulated emission [252]. SOAs are important for telecommunication wavelengths, but recent attention has been given to visible SOAs in support of the development of new technology platforms such as MOPAs [236] and swept sources [253]. Integration of an SOA alongside a laser in a chip opens a pathway for realizing visible-range photonic integrated circuits that could exhibit immunity to the intrinsic optical loss of visible-light waveguides [254].

To this end, Shen et al reported an integrated laser-SOA based on an InGaN-based laser $(\lambda=404 \mathrm{~nm})$ grown on a semipolar (2021) GaN substrate in 2018 [206] (see figure 14). A nonlinear gain of $5.7 \mathrm{~dB}$ was achieved upon applying $6.25 \mathrm{~V}$ to the amplifier section. The $2 \mu \mathrm{m}$ wide ridge-waveguide laser, which had a $300 \mu \mathrm{m}$ long SOA section integrated in front of a $1190 \mu \mathrm{m}$ long gain section, gave a maximum output optical power of $30.5 \mathrm{~mW}$.

In 2012, Stanczyk et al reported a single top-contact device ( $\lambda \sim 408 \mathrm{~nm}$ ) composed of a $3 \mu \mathrm{m}$ straight ridge-waveguide section (between $150 \mu \mathrm{m}$ and $325 \mu \mathrm{m}$ long) and a flared waveguide section used for amplification (between $325 \mu \mathrm{m}$ and $500 \mu \mathrm{m}$ long with a taper angle between $2^{\circ}$ and $5^{\circ}$ ) [212]. This configuration produced a maximum optical output power of $600 \mathrm{~mW}$.

While the integration of the SOA-laser is an attractive topic, gain saturation challenges should be addressed. Gain saturation is due to the inability of the SOA to continuously amplify incoming light at increasing optical power levels. This is related to the rate at which carriers can be injected into the SOA while the photon density increases along the SOA. This problem affects SOAs and superluminescent diodes (section 2.3) alike [255, 256].

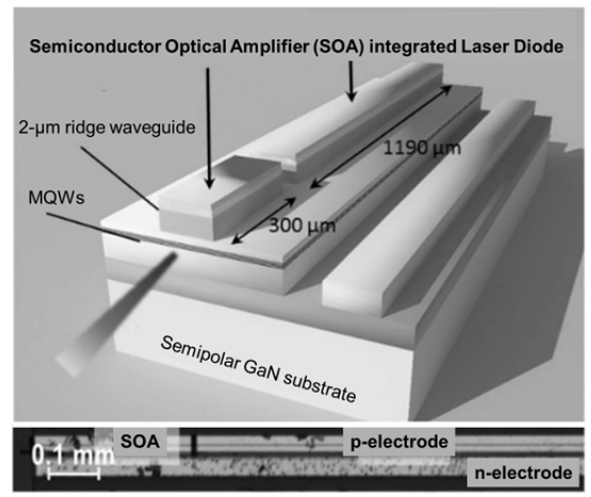

Figure 14. Schematic and microscope images (inset below) of an SOA integrated laser diode implemented using a $2 \mu \mathrm{m}$ ridge-waveguide structure and a semipolar (2021) GaN substrate. The dimensions of the amplifier and gain sections are indicated accordingly. Adapted with permission from [206]. (C) The Optical Society.

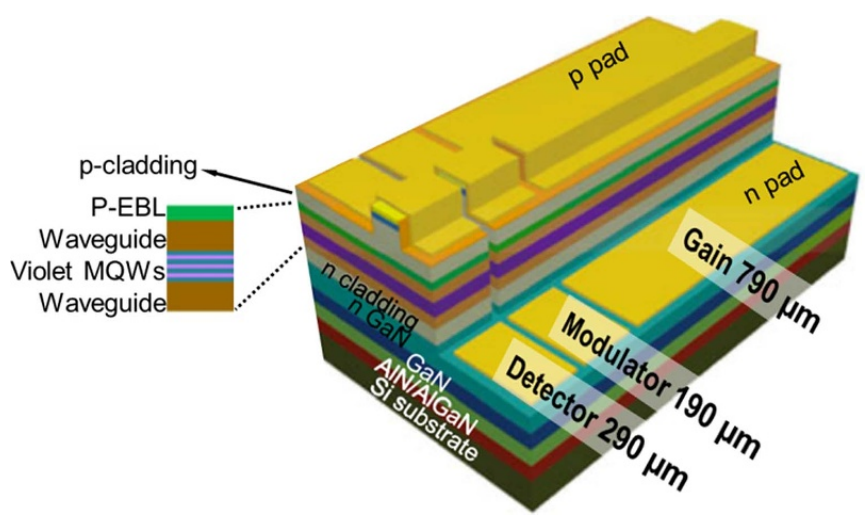

Figure 15. Schematic of a device in which the detector and modulator are integrated with the gain section to form a three-section laser diode. The dimensions of the various sections and layer structures are indicated. (C) 2018 IEEE. Reprinted, with permission, from [211].

\subsubsection{Integrated laser-photodetector. A necessary step} towards multicomponent integration and the development of photonic integrated circuits based on $\mathrm{InGaN} / \mathrm{GaN}$ is the study of integrated laser-photodetectors, which can aid in monitoring the operation of laser diodes in stand-alone integrated devices and photonic integrated circuits. This was investigated by Shen et al in 2017 using a semipolar (20 $\overline{21})$ GaN-based platform $(\lambda=405 \mathrm{~nm})$ [257] with a photodetector responsivity of $0.051 \mathrm{~A} \mathrm{~W}^{-1}$ at $-10 \mathrm{~V}$ and a modulation bandwidth of $230 \mathrm{MHz}$. This strategy was further explored in 2018 by Feng et al $(\lambda \sim 413 \mathrm{~nm})$ [211], who demonstrated a three-section gain-modulator-photodetector configuration (see figure 15). This device was demonstrated using a $c$-plane InGaN/GaN laser structure grown on a silicon substrate, and could potentially offer a low-cost, large-scale implementation. The photodetector exhibited a responsivity of $\sim 0.015 \mathrm{~A} \mathrm{~W}^{-1}$ at $-4 \mathrm{~V}$.

The fact that the photodetector section shares the epitaxial structure with the laser and modulator provides a significant device integration advantage. In contrast, traditional silicon photonics and photonic integrated circuits require individual 
growth of the laser source and photodetector, followed by device transfer [258]. This increases the complexity of largescale integration.

\subsubsection{Integrated laser-optical element: air/semiconductor} mirrors and diffraction grating. The monolithic integration of passive optical elements with a laser diode via a single etching step is attractive for large-scale photonic integration. A small footprint can be achieved by forming passive optical elements in the same semiconductor structure as the laser.

Air-semiconductor DBRs (figure 16(a)) were initially developed to improve reflectivity at laser facets and thus reduce the threshold current and improve the lifetime of the GaN laser. This approach is important for lasers that are integrated into monolithic photonic circuits where it is difficult to implement facet cleaving and dielectric mirror coatings. Available reports discuss discrete laser-DBR integration ( $\lambda$ range of 400-407 nm) [214, 259-264], but further investigation is required at the level of photonic circuit integration. Similarly, integration of laser diodes with a special type of diffraction grating enables vertical emission from in-plane lasers at $\lambda$ of $407 \mathrm{~nm}$ (see figure 16(b)) [213, 265]. This can be attractive for board-to-board free-space data transfer, as well as diagnostics of processes that occur within a photonic integrated circuit and post-fabrication device testing.

\subsection{Narrow-linewidth laser via integration of a DFB grating}

\subsubsection{Overview. DFB gratings were demonstrated using} InGaN-based lasers to overcome low reflectivity at the facets during early development [266]. Now, DFB lasers are being designed to achieve single-frequency operation. Ideally, DFB gratings would selectively diffract light from the laser diode back into the cavity based on the Bragg condition [267] and the chosen dominant wavelength or frequency. DFB lasers differ from the above-mentioned multi-section lasers because the DFB grating is incorporated within the gain section instead of being a separate section. The DFB grating can be implemented near the active region as a buried grating (e.g. $80 \mathrm{~nm}$ period and $100 \mathrm{~nm}$ depth) via electron-beam lithography, inductively coupled plasma etching, and subsequent overgrowth. A $405 \mathrm{~nm}$ DFB laser (see figure 17(a)) based on such a fabrication scheme utilized a first-order grating to achieve $\mathrm{CW}$ operation at $25{ }^{\circ} \mathrm{C}$ showing a threshold current and slope efficiency of $22 \mathrm{~mA}$ and $1.44 \mathrm{~W} \mathrm{~A}^{-1}$, respectively [268]. This first GaN-based continuous-wave DFB laser determined the goals of later efforts.

Alternately, a DFB grating can be formed on the surface of a laser device but outside of the p-contact stripe, as shown in figure 17(b). The scanning electron microscope (SEM) images show the DFB grating in the form of V-shaped grooves fabricated beside the $p$-contact stripe. The violet $(\lambda$ of approximately $405-410 \mathrm{~nm}$ ) laser sustained CW operation and was based on a 10th order laterally coupled surface grating [269].

To put things in perspective, buried gratings represent a significant challenge for the already complex growth of groupIII-nitride lasers. This has driven the use of surface gratings (a)

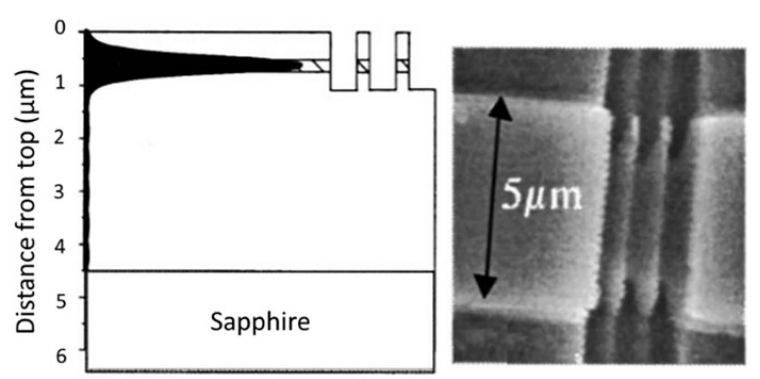

(b)

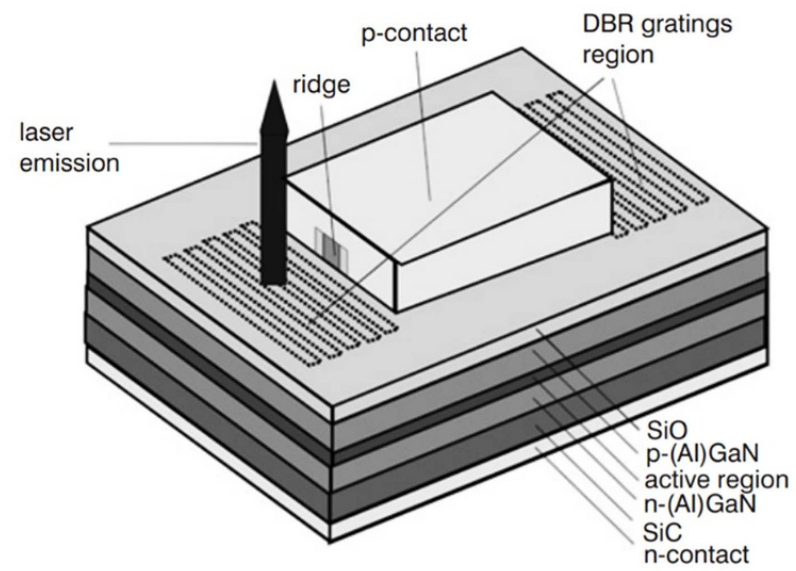

Figure 16. (a) Device schematic and SEM image of an air/semiconductor DBR-integrated edge-emitting laser on a sapphire substrate. Adapted from [260], with the permission of AIP Publishing. (b) Schematic of integrated laser-DBR grating designed to achieve vertical laser emission. Green and orange colors indicate the $\mathrm{SiO}_{2}$ layer and top or bottom electrodes, respectively. Epitaxial layer structures and the $\mathrm{SiC}$ substrate are indicated accordingly. Republished with permission of the Institution of Engineering and Technology (IET), from [213], permission conveyed through Copyright Clearance Center, Inc.

as the dominant grating technology in recent years. Together with surface gratings, higher order gratings have also been adopted to relax fabrication requirements. Together, these two approaches have led to the successful demonstration of highperformance DFB lasers and are expected to remain relevant.

2.2.2. Device performance. Since 1996, the development of InGaN-based DFB lasers has produced improved electrical operation, narrower linewidths, increased side-mode suppression ratios (SMSRs), and higher optical output power [268-295]. Most studies have focused on violet-to-blue emission ( $\lambda$ of approximately $400-450 \mathrm{~nm}$ ) due to material availability, although recent reports have addressed sky-blue $(\lambda$ of approximately $480 \mathrm{~nm}$ ) [288] (see figure 18) and green ( $\lambda$ of approximately $514 \mathrm{~nm}$ ) lasers [287, 296]. These recent efforts indicate strong progress in the development of DFB single-frequency emitters. CW operation has become the norm [269, 278, 282, 287, 288], with output power as high as $\sim 90 \mathrm{~mW}$ (continuous wave) [269] and $200 \mathrm{~mW}$ (pulsed) [286], as well as SMSRs as high as $\sim 42 \mathrm{~dB}$ [288] (see figure 18). It is important to mention that measurement of 
(a)



(b)

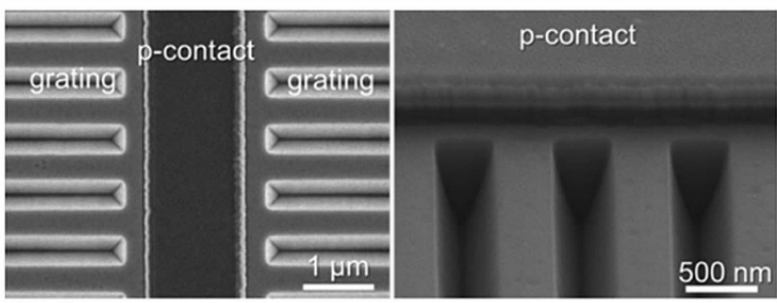

Figure 17. (a) Device schematic of a $405 \mathrm{~nm}$ DFB laser with a first-order grating implemented on an n-type cladding layer (buried grating with an $80 \mathrm{~nm}$ period and $100 \mathrm{~nm}$ depth via electron-beam lithography). Adapted from [268]. (C) IOP Publishing Ltd. All rights reserved. (b) Scanning electron microscope images of 10th order laterally coupled surface gratings in the form of V-shaped grooves fabricated beside the p-contact stripe of a violet (approximately $405-410 \mathrm{~nm}$ ) laser diode. Adapted with permission from [269]. (C) The Optical Society.

the linewidths of InGaN-based DFB lasers has not been well established, as it mostly relies on the resolution provided by optical spectrum analyzers. Characterization of the real linewidth is a necessary step towards understanding GaN DFB laser noise and frequency stability. This could lead to the eventual implementation of such compact devices in frequency standards and atomic clocks. A comprehensive report on the wavelengths and linewidths required for such applications can be found in [297].

DFB lasers at visible wavelengths are aimed at enabling compact solutions for atomic clocks, spectroscopy, and optical communication. DFB lasers provide a monolithic solution capable of being deployed in space or underwater probes

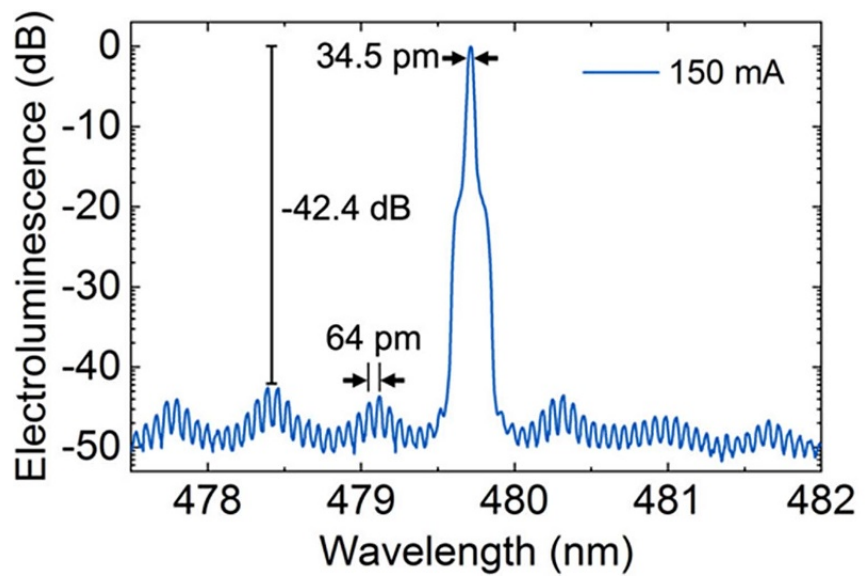

Figure 18. The characteristic emission spectrum of an InGaN-based DFB laser diode exhibits a single lasing mode centered at approximately $480 \mathrm{~nm}$ with an SMSR of approximately $42 \mathrm{~dB}$. High-speed optical wireless communication of up to $10.5 \mathrm{Gbit} \mathrm{s}^{-1}$ was demonstrated using such a laser in [288, 298]. Adapted with permission from [288]. () The Optical Society.

where small device sizes and immunity to vibration are important. A recent demonstration of high-speed optical communication (up to $10.5 \mathrm{Gbit} \mathrm{s}^{-1}$ ) using an InGaN-based DFB laser shows the potential for further development of these devices for data transmission and fast modulation [288, 298].

\subsection{Superluminescent diodes and ASE}

2.3.1. Overview. Superluminescent diodes are light sources that operate in the ASE regime [299], in which the amplification of spontaneous emission is achieved via stimulated emission, such as in laser diodes, but restricted by a lack of optical feedback. This lack of optical feedback restraining the resonating cavity is the main difference between the laser diode and both superluminescent diode and SOA. The superluminescent diode amplifies its own generated light, whereas an SOA merely amplifies incoming external light; nonetheless, their epitaxial structure can be identical. Superluminescent diodes achieve stimulated emission by utilizing a waveguide epitaxial structure equivalent to that of a laser diode, where spontaneous emission from the heterostructure is guided and amplified within the device. Disruption of the optical feedback is achieved by reducing the facet reflectivity using any of several methods including tilted facets [300], antireflection (AR) facet coatings [301], waveguide bending [302], passive absorbers [204], and facet roughening [303]. A combination of broad spectral emission, droop-free operation, and a high optical power density can then be achieved to support applications in optical coherence tomography [304] and fiber gyroscopes [305].

For ASE to occur, there must be sufficient material gain to overcome material losses (e.g. non-radiative recombination and absorption). One way to exploit this material gain is by using a double-pass superluminescent diode configuration, which relies on a cavity where the reflectivity of one facet is 
enhanced by a dielectric DBR coating. Thus, the optical power $\left(P_{o}\right)$ is proportional to the square of the optical gain $\left(G_{s}\right)$ [306]:

$$
P_{\mathrm{o}} \approx P_{\mathrm{sp}} R G_{\mathrm{s}}^{2},
$$

where $P_{\mathrm{sp}}$ is the power of the spontaneous emission, $R$ denotes the reflectance of the high-reflection DBR mirror, and $G_{\mathrm{s}}$ is the single-pass optical gain. The value of $G_{\mathrm{s}}$ is related to the current density $J$ and cavity length $L$, as follows [306]:

$$
G_{\mathrm{s}}(J, L)=\exp \left[\left(\Gamma g_{\mathrm{o}} \eta \frac{J}{d}-\alpha\right) L\right],
$$

where $\Gamma$ is the confinement factor, $g_{0}$ is the gain of the active region, $\eta$ denotes the internal quantum efficiency, $d$ is the thickness of the active layer, and $\alpha$ is the modal absorption. Since the device length and current density can be adjusted simply, the optimal regime of operation can be achieved in standard epitaxial structures. Nonetheless, we should remember the gain saturation regime (similar to that of an SOA), where the photon density is higher than the carrier density and thus the amplification process is hindered [255, 256]. In this case, epitaxial engineering is needed to improve other parts of equation (3).

\subsubsection{Device performance and applications. InGaN-based} superluminescent diodes offer characteristics such as low speckle noise, large modulation bandwidth, high optical power density, and droop-free operation at short wavelengths [250, $256,307,308]$. Since the first demonstration of GaN superluminescent diodes in 2009 [309], they have been included in applications such as white lighting [248] and visible-light communication [251, 310, 311]. Furthermore, to fulfill the needs of picoprojectors, sensing, and biomedicine, consistent research has been performed to enhance the optical bandwidth, output optical power, and emission at different wavelengths (e.g. near ultraviolet, blue, and green light) from $\mathrm{GaN}$ superluminescent diodes [307, 312-314].

The highest optical power from a CW InGaN-based superluminescent diode is $350 \mathrm{~mW}$ recorded for a ' $\mathrm{j}$-shaped' curved-waveguide superluminescent diode that emits light at $405 \mathrm{~nm}$ and was reported by Castiglia et al [315]. In contrast, the highest obtained optical power of a pulsed superluminescent diode is $2.2 \mathrm{~W}$, as reported by Cahill et al, who fabricated the superluminescent diode on a $c$-plane GaN-substrate that emits blue light at $416 \mathrm{~nm}$ [316]. This is a horizontal device that uses deflecting mirrors to produce vertical emission.

The highest superluminescent diode modulation bandwidth reported is $2.5 \mathrm{GHz}$ by Rashidi $e t a l$ and was produced using a non-polar GaN substrate [317]. The leading role of non-polar $\mathrm{GaN}$ in achieving record modulation bandwidths is likely to be maintained [217]. The shortest device cavity was reported by Zhang et al, who demonstrated a $40 \mu \mathrm{m}$ long superluminescent diode [318] with a significantly broadened emission $(15 \mathrm{~nm})$ but limited in optical power $(\sim 2 \mathrm{~mW})$, as expected, due to its short device length.
The first report of a GaN-superluminescent diode grown on silicon was presented by Liu et al [319]. It provided a path for integrating monolithic silicon photonics and $\mathrm{GaN}$ sources. However, heteroepitaxy remains a challenge to provide the optical output power levels of such devices. A $15.5 \mathrm{~nm}$ broadest full-width at half-maximum (FWHM) was recorded from an InGaN violet superluminescent diode designed by Kafar et al using the concept of a non-linear indium content profile along the superluminescent diode waveguide [320]. This approach can provide a way to extend the limited optical bandwidths of GaN superluminescent diodes (usually 5-8 nm), but difficulties with optical absorption due to self-pumping of the active region remain to be solved.

In the latest development in green-emitting superluminescent diodes, Primerov et al at EXALOS demonstrated the provision of up to $10 \mathrm{~mW}$ of superluminescent diode light at $517 \mathrm{~nm}$ (targeted for picoprojection applications) [321, 322]. The production of such a green-emitting device indicates a level of GaN superluminescent diode design and fabrication maturity and the ability to overcome the drop in efficiency, reduced optical confinement, and related material issues that occur at longer wavelengths.

The future of GaN superluminescent diodes is affected by material constraints that affect device efficiency limitations that in turn affect green emission. Despite this, truegreen superluminescent diodes may play a significant role in the upcoming augmented reality and virtual reality revolution, which includes consumer electronics. InGaN QDs [314] seem to offer a relevant development path, although this concept has barely been explored. Blue superluminescent diodes with increased modulation bandwidths will be necessary for smart lighting applications. Engineering approaches aimed at increasing energy efficiency [323] may eventually accelerate large-scale market adoption.

Readers are further referred to a review article by Shen $e t a l$, which provides a detailed account of progress in InGaN-based superluminescent diodes [307].

\section{Group-III nitride and halide perovskite lasers and ASE towards the green-gap}

Recent reports on the use of halide perovskite as an optical gain medium that can exhibit ASE and lasing action bear testament to its potential for producing 'green-yellow gap' lasers. Such lasers are currently difficult to produce efficiently using group-III nitride or group-III phosphide-arsenide material systems due to lattice and thermal mismatches and physical limitations related to bandgap structures. Ease of solution processability can reduce the cost of fabrication relative to conventional epitaxial growth methods. Most importantly, while InGaN- and AlGaInP-based lasers have correspondingly been demonstrated to cover the $375-530 \mathrm{~nm}$ wavelength region and the red wavelength region at $>630 \mathrm{~nm}$, respectively, they are inadequate for efficient operation in the green-yellow wavelength range, which remains a topic of research focus [324]. 




Figure 19. Combinations of laser and superluminescent diode devices with varying gain-medium constituents. Blue and green colors represent devices with only group-III-nitride- or perovskite-based gain media, respectively. Transitional blue-green colors indicate the utilization of both semiconductors in a heterogeneous, integrated manner.

\subsection{Towards hybrid integration}

As noted in figure 19, laser and ASE devices can be implemented using horizontal and vertical waveguide configurations. The laser and superluminescent diodes described in section 2 mostly focus on horizontal emission made up of group-IIInitride semiconductors on sapphire or GaN substrates. Using such a horizontal waveguide configuration and $\mathrm{SiO}_{2} / \mathrm{TiO}_{2}$ DBRs, nanowire was grown on a silicon substrate and fabricated into a laser emitting at $533 \mathrm{~nm}$ [325].

In addition, a VCSEL [326, 327] and ASE device or random laser based on a nanowires ensemble [328, 329] were demonstrated on hybrid-dielectric or all-dielectric DBRs, as well as photonic crystal $(\mathrm{PhC})$ elements. Apart from that, Br-based perovskite can be integrated with DBRs $[330,331]$ or porousGaN/GaN DBRs [332] to produce vertical emission devices, as discussed below.

Despite the rapid development of halide-perovskite-based materials as preferred gain media, heterogeneous integration between solution-processed perovskite films and existing device technology, e.g. in-plane and VCSELs, remains a major challenge. In particular, although optically pumped VCSELs realized based on all-dielectric DBR structures have been widely reported in recent years, it is still challenging technologically in implementing electrically-conducting DBR structures, which would allow current-injection devices. In 2017, Chen et al [332] were among the earliest to produce a perovskite-based optically pumped VCSEL based on $\mathrm{CH}_{3} \mathrm{NH}_{3} \mathrm{Pbl}_{3}$ thin films and a custom-designed GaN-based high-quality-factor optical resonator. Here, we highlight an encouraging work based on nanoporous-GaN VCSEL with a bottom conductive DBR structure [333], which could be promising in future electrically injected halide-perovskite VCSELs if successfully integrated.

Lattice-matched nanoporous-GaN (NP-GaN) and GaN stacks are deposited as a DBR structure on the VCSEL in figure 20(a), and layered with the perovskite thin films. The VCSEL structure exhibits a single-mode lasing peak at $778.4 \mathrm{~nm}$ (see figure 20(b)), an SMSR of more than $20 \mathrm{~dB}$, and a low threshold density of $113.9 \mu \mathrm{J} \mathrm{cm}^{-2}$. Instead of relying on (a)

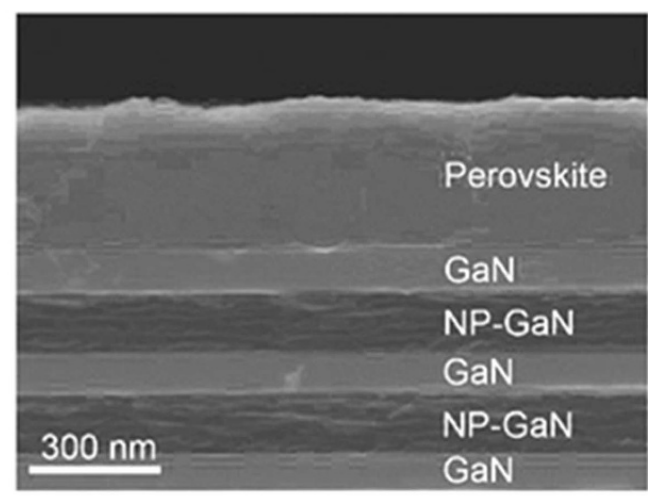

(b)



Figure 20. (a) SEM image of a perovskite-based thin film with a lattice-matched NP-GaN/GaN DBR structure intended for perovskite-based VCSEL. (b) Single-mode lasing at $778.4 \mathrm{~nm}$ with an SMSR that exceeds $20 \mathrm{~dB}$. [332] John Wiley \& Sons. Copyright (C) 2017 WILEY-VCH Verlag GmbH \& Co. KGaA, Weinheim.

dielectric DBR structures typically consisted of oxide-based layers, this represents a significant step towards monolithic integration of a perovskite-based cavity medium with a nitridebased DBR to produce an electrically pumped VCSEL. This effort seeks to address the scarcity of visible light-based lasers, and in particular the 'green-gap' in conventional group-IIInitride-based materials. Moreover, by simply substituting the halide-anions in the perovskite gain medium, this would allow reconfigurable, solution-processable and wavelength-tunable light-emitting devices without the need of considering the issues of lattice-mismatch, which remained a key consideration in group-III-nitride growth processes.

Although the integration of perovskite with group-IIInitride materials is still not well studied, it would be of great interest to explore multifunctional properties available via hybrid structures and potentially open up a new avenue for novel applications. Along with the development in this regard, there remain a few key challenges that need to be resolved. For instance, it has been noted that perovskite might suffer from poor thermal dissipation under high electrical injection [128] and could possibly degrade the long-term performance of the lasing devices due to the sensitivity of the perovskite gain medium to the heating effect. Thus, integration of GaN-based 
DBRs on bulk-metal substrates may potentially relieve heating issues and extend the lifetimes of future electrically driven perovskite-based laser devices. In terms of device fabrication and processability, the dissociation of perovskite structure under external heating $\left(>150{ }^{\circ} \mathrm{C}\right)$ could be challenging for integration with nitride-based DBR structures, as the deposition of group-III-nitride-based materials requires a higher growth temperature of up to $>500{ }^{\circ} \mathrm{C}$.

A recent demonstration by Kelvin et al [334] has also provided a promising perspective on the formation of composite structures from $\mathrm{MAPbBr}_{3}$ and nanoporous GaN. Infiltration of $\mathrm{MAPbBr}_{3}$ into the highly porous $\mathrm{GaN}$ layer via a low-temperature post-processing step may enhance spatial confinement in the perovskite within the encapsulation matrix while preserving perovskite photostability [335]. Although this remains to be demonstrated, such a composite may be promising with regard to monolithic integration of phosphorLED technology, as well as for its potential for random lasing due to its improved quantum confinement effect.

\subsection{Perovskite lasing and ASE}

In addition to their inherent high defect tolerances, high absorption coefficients, high exciton binding energies, and high PLQYs, semiconducting metal-halide perovskites exhibit promise for development as optical gain materials [336-338]. In particular, perovskite-based materials may be used for lowcost optoelectronic devices operating in the green-yellow gap in which the conventional group-III-nitride-based materials face significant challenges, as well as offering an alternative approach for wavelength-tunable visible lasers in which solution-processed perovskite is used as an optical gain medium. This section summarizes the recent development of perovskite-based materials as a gain medium for optically pumped devices. We further provide perspectives on the choice of gain medium and design structures.

An earlier study of perovskite-based gain media for ASE and lasing devices was performed by Xing et al in 2014 [339]. Despite the low PLQY demonstrated, the solution-processed perovskite-based gain media (e.g. $\mathrm{MAPbCl}_{3}, \mathrm{MAPbCl}_{1.5} \mathrm{Br}_{1.5}$, $\mathrm{MAPbBr}_{3}, \mathrm{MAPbBrI}_{2}$, and $\mathrm{MAPbI}_{3}$ ) exhibit ASE across the entire visible wavelength region (i.e. 390-790 nm) through simple halide substitution, as shown in figure 21 . In the subsequent year, Priante et al [340] demonstrated true-green ASE at $553 \mathrm{~nm}$ using $\mathrm{MAPbBr}_{3}$ as an optical gain medium. The emission spectra of $\mathrm{MAPbBr}_{3}$ thin films are correlated to bulk defect states that have also been shown to generate radiative energy levels and lead to strong ASE. This work represents an alternative path for replacing InGaN-based platforms for emission at these wavelengths, particularly low indium incorporation efficiency in MOCVD-grown commercial LEDs, high lattice mismatch, and excessive threading dislocations that lead to low EQEs of typically less than $20 \%$.

After these initial studies, a significant number of recent works also demonstrated optically pumped ASE and lasing characteristics using a perovskite platform with an emphasis on the green-emitting region. Studies have also demonstrated

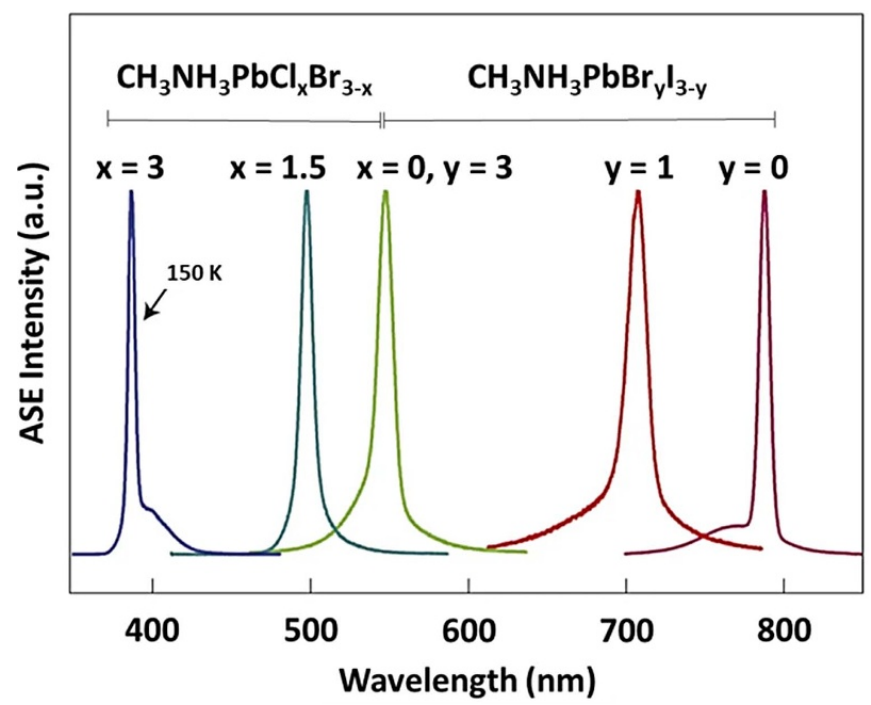

Figure 21. Tunable ASE wavelengths produced using perovskites with various halide substitutions as gain media. All spectra were measured at room temperature, except for $\mathrm{CH}_{3} \mathrm{NH}_{3} \mathrm{PbCl}_{3}$, which was measured at a lower temperature of $150 \mathrm{~K}$. Adapted by permission from Springer Nature Customer Service Centre GmbH: Springer Nature, Nature Materials [339], () 2014.

various design structure improvements, e.g. dimensional confinement, active region design, and surface-passivated gain media.

Table 3 summarizes recent progress in ASE and lasing using perovskite-based gain media. The table focuses on advances that target the true-green wavelength, i.e. $555 \mathrm{~nm}$. Hybrid organic-inorganic perovskites based on $\mathrm{MA}^{+}$and $\mathrm{FA}^{+}$ cations are the dominant choice of gain medium in early works starting around 2015. However, inorganic perovskites based on $\mathrm{Cs}^{+}$cations have received progressively increased attention due to their greater tolerance for thermal-, moisture-, and photo-induced degradation relative to their organic-inorganic counterparts [145, 341]. Along with rapid advances in surface passivation [155, 342], core/shell structures [157], and the use of low-dimensional nanostructures with high confinement factors (e.g. QD and nanowires) [343, 344], the pumping thresholds of high-performance perovskite-based gain media have decreased from hundreds to few $\mu \mathrm{J} \mathrm{cm}^{-1}$ over a mere 4 year period. Moreover, at the current stage of development, further progress towards lowering the ASE and lasing thresholds will undoubtedly require an external optical feedback structure, e.g. DBR mirrors based on dielectric materials (e.g. $\mathrm{SiO}_{2}, \mathrm{Si}_{3} \mathrm{~N}_{4}, \mathrm{Al}_{2} \mathrm{O}_{3}, \mathrm{HfO}_{2}$ ) [330-332, 345] and stamped DFB gratings on the perovskite-based active medium $[135,346]$. Although lithographically defined perovskitebased superluminescent diodes and lasers remain a challenge, a recent demonstration of CW-pumped ASE and lasing [330] lays a strong foundation for the fabrication of multi-section devices that are critical to monolithic integration for various commercial applications and progress towards electrically injected halide-perovskite-based laser diodes. Importantly, optical gains related to $\mathrm{Br}$-based nanorods have also been characterized at up to $980 \mathrm{~cm}^{-1}$ using the variable stripe 







\section{Demonstrated strategies towards ASE and lasing in perovskite-based gain media}

\section{Strategies}

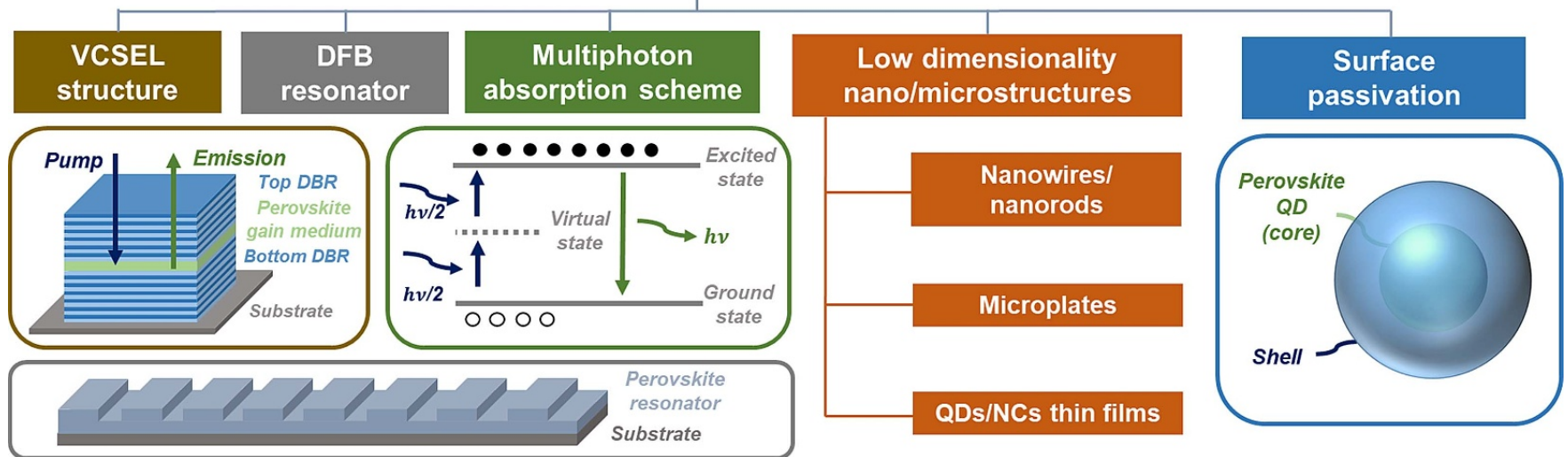

Figure 22. A summary of demonstrated strategies for ASE and lasing in perovskite-based gain media.

length method by Wang et al [343]. This suggests an approach to further lowering lasing thresholds and enhancing the efficiencies of future electrically injected perovskite-based laser diodes [347].

Figure 22 summarizes the strategies commonly employed to achieve ASE and lasing in halide-perovskite-based gain media, including vertical VCSEL or horizontal DFB resonator schemes. The strategies are somewhat similar in concept and implementation to group-III nitride nanophotonics approaches [360]. Further, the existing work focused on optically pumped devices by using femtosecond-based lasers, including multiphoton-absorption pumping, to achieve ASE and lasing. Some devices were even operated under cryogenic conditions, which limits their practical implementation. Low dimensionality nanostructures and surface passivation are also employed to ensure high PLQY and lasing operation. The following summarizes and discusses these strategies:

3.2.1. VCSEL structure. CW optically pumped lasing system, with sustained population inversion and lasing, is a prerequisite for electrically driven laser diodes [361, 362]. For room temperature operation, in 2017, Alias et al [330] demonstrated CW optically pumped, green-emitting $\mathrm{MAPbBr}_{3}$ VCSEL with a peak wavelength of $541 \mathrm{~nm}$. As shown in figure 23(a), the optically pumped perovskite-based VCSEL is formed by sandwiching an active region consisting of $\mathrm{MAPbBr} 3$ gain media with a $\mathrm{SiO}_{2} / \mathrm{Si}_{3} \mathrm{~N}_{4} \mathrm{DBR}$ structure and the PMMA layers. Figure 23(b) shows lasing characteristics with an enhanced integrated intensity and a significantly reduced FWHM above the CW pumping threshold of $89 \mathrm{~kW} \mathrm{~cm} \mathrm{~cm}^{-2}$. For improved thermal dissipation, a singlecrystal sapphire was used as the substrate during the measurement. For the development of an electrically driven perovskitebased laser, it is critical to demonstrate the optical gain under uncooled $\mathrm{CW}$ operation at room temperature or at lower temperatures achievable via Peltier cooling for future electrically pumped configurations [347, 363]. (a)

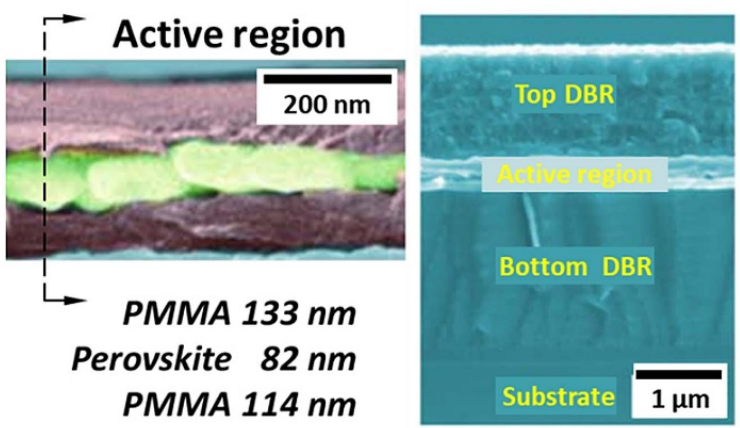

(b)

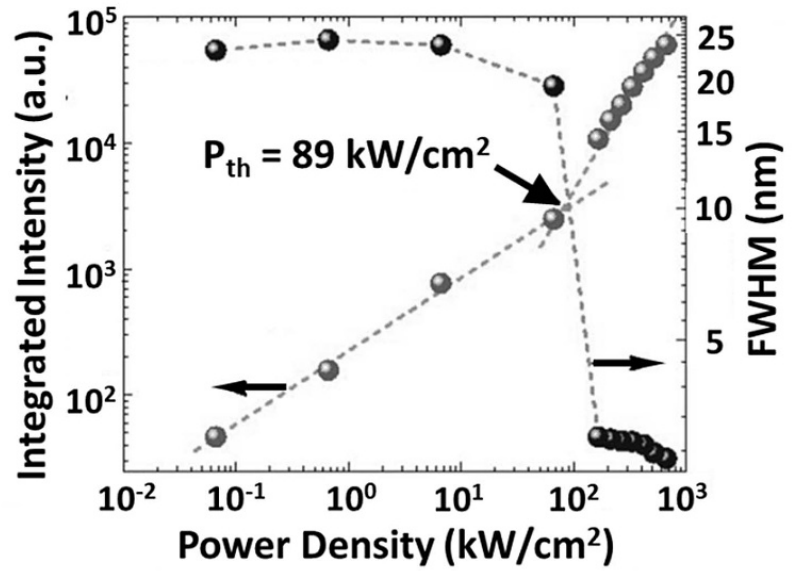

Figure 23. (a) Scanning electron microscopy images showing a cross-section of the CW-pumped, green-emitting perovskite-based VCSEL with the active region thicknesses indicated. The active region is sandwiched between the top and bottom DBRs and the device is prepared on a sapphire substrate. (b) Integrated PL intensity and FWHM of a VCSEL under CW excitation by a $325 \mathrm{~nm}$ $\mathrm{He}-\mathrm{Cd}$ laser with an optical power of $\sim 8 \mathrm{~mW}$. Adapted with permission from [330]. () The Optical Society.

For practical applications, such as high-speed optical fiber communication [364], atomic clocks [365], and sensing [366], single-mode lasing is required because of its high spectral purity and beam quality. While each of these applications 
may require a specific laser linewidth depending on a specific situation, useful linewidths are regularly found to be a few $\mathrm{MHz}$ for optical communication [364]; sub-MHz to sub$\mathrm{Hz}$ for atomic clocks [365]; and sub-MHz for high-resolution sensing [366]. Although most prior work has produced evidence of ASE and lasing in halide-perovskite-based gain media, various results indicate random or multi-mode lasing with high pumping thresholds. This is due to several challenges, including a random distribution of gain media that results from irregular morphology, defect sites on the gain medium, and poor interfaces.

To obtain high-quality coherent beams and single-mode lasing, in 2016, Chen et al [367] embedded red-emitting $\mathrm{MAPbI}_{3}$ thin films with 2D PhC resonator, which is also employed in group-III nitride semiconductor diode lasers. Temporally coherent single-mode lasing in the green-emitting yet flexible $\mathrm{FAPbBr}_{3}$-based VCSEL was also demonstrated by Chen et al [331] in 2017. Polycrystalline $\mathrm{FAPbBr}_{3}$ thin films were sandwiched between sputtered dielectric $\mathrm{HfO}_{2} / \mathrm{SiO}_{2}$ DBR structures, which naturally form much-needed encapsulation layers for enhanced lasing lifetimes (i.e. up to $\sim 20 \mathrm{~h}$ or equivalent to $\sim 10^{8}$ laser shots) under ambient conditions. For the flexible VCSEL, temporally coherent single-mode lasing is still observed with a peak wavelength of $552.7 \mathrm{~nm}$. Even though the achieved quality factor $(Q)$ of $\sim 920$ in the flexible substrate is lower than that in the rigid substrate $(\sim 1420)$, the ease of fabrication highlights the potential for solutionprocessable perovskite-based gain media to be used in flexible, single-mode laser devices. In comparison, a lift-off and fabrication process for group-III-nitride-based VCSEL is much more elaborate and challenging.

3.2.2. $D F B$ resonator. To realize compact and highperformance perovskite-based emitters, the lithographydefined perovskite-based structures remained a critical issue due to the structural instability upon exposure to the ambient environment and other processing solvents; this, in particular, hinders the realization of resonator structures. It was until around 2018, where Pourdavoud et al fabricated secondorder DFB resonator gratings with a periodicity of $300 \mathrm{~nm}$ formed on $\mathrm{MAPbBr}_{3}$ thin film via thermal nanoimprinting, as shown in figure 24(a). The work demonstrated tunable lasing in the wavelength region between $543 \mathrm{~nm}$ and $557 \mathrm{~nm}$, with a low lasing threshold of $\sim 3.4 \mu \mathrm{J} \mathrm{cm}^{-2}$ [135]. Due to the sensitivity of perovskite-based materials to various commonly used solvents in wet-chemical lithography, the formation of high-Q resonators had thus far been limited to direct thermalnanoimprinting on perovskite thin film using pre-patterned $\mathrm{SiO}_{2}$ mold [346] or linear photonic grating [135]. Using cavities formed by this method, optically pumped ASE and lasing actions have been observed. For instance, a shallow grating imprinted onto $\mathrm{MAPbI}_{3}$ thin film results in a 16-fold reduction in linewidth from $38 \mathrm{~nm}$ to $2.4 \mathrm{~nm}$, indicating ASE, as shown in figure 24(b).

3.2.3. Multiphoton absorption scheme. While singlephoton absorption (1 PA) schemes are widely used to obtain

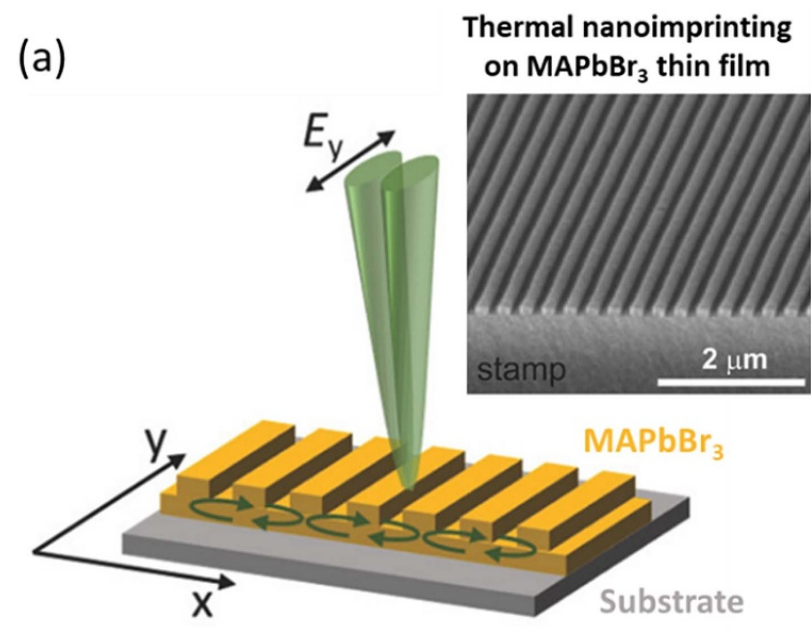

(b)

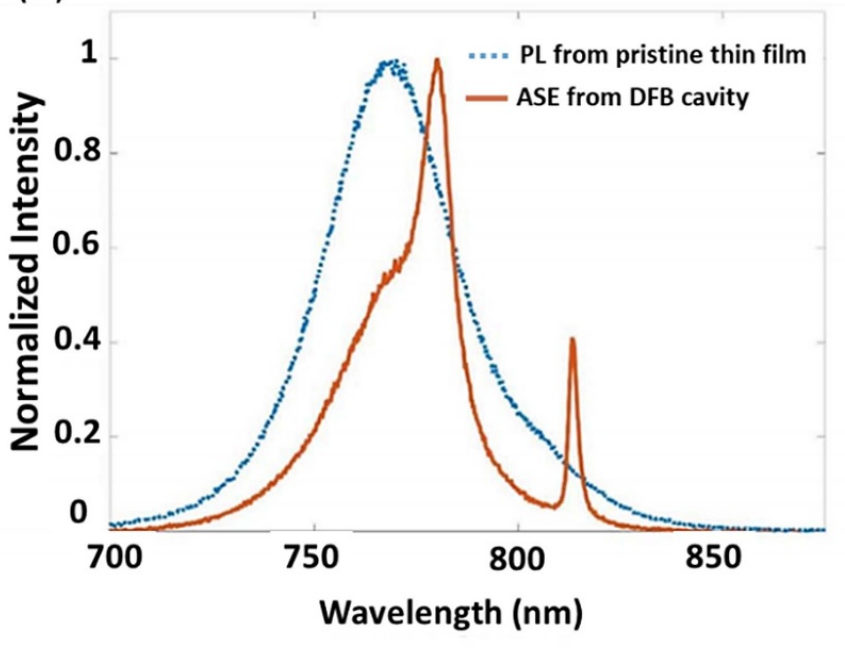

Figure 24. (a) Schematic diagram of DFB resonator formed on optically pumped $\mathrm{MAPbBr}_{3}$-based devices with the assignment of pump direction and laser emission normal to the sample surface. The inset shows the thermal-nanoimprinted gratings with a periodicity of $300 \mathrm{~nm}$ formed onto the active medium of $\mathrm{MAPbBr}_{3}$. [135] John Wiley \& Sons. Copyright (C) 2018 WILEY-VCH Verlag GmbH \& Co. KGaA, Weinheim. (b) Comparison between the normalized spectra of pristine $\mathrm{MAPbI}_{3}$ thin film (see dotted blue line) and with the thermal-nanoimprinted DFB cavity (see solid red line). The narrowing of linewidth to $2.4 \mathrm{~nm}$ with the incorporation of the DFB cavity indicating ASE. Adapted with permission from [346]. () The Optical Society.

the desired ASE and lasing phenomena, the non-linear optical properties of perovskite-based gain media that use multiphoton (two- to six-photon) absorption have also been explored. Multiphoton absorption minimizes photoinduced damage, increases penetration depths, and increases spatial resolutions [155, 348, 368]. Via their non-linear absorption properties, perovskite-based gain media may potentially produce an alternative path towards applications in high-capacity data storage [369, 370], bioimaging [371, 372], photodynamic therapy [373], and multiphoton lithography [374]. In 2015, Pan et al [155] demonstrated the first highly stable ASE based on two-photon absorption (2 PA) with surface-passivated, solution-processed $\mathrm{CsPbBr}_{3}$ QDs. The ASE intensity with 
an excitation threshold of $12 \mathrm{~mJ} \mathrm{~cm}{ }^{-2}$ remained stable over $1.2 \times 10^{8}$ laser shots. These prior works highlight the potential of emerging perovskite-based materials as frequencyupconverted optical gain media for non-linear optoelectronic devices. They may serve to complement non-linear waveguides based on group-III-nitride materials [375, 376] under photonic chip integration schemes.

3.2.4. Low dimensionality nano/microstructures. In addition, while the ASE or lasing characteristics of primarily two-dimensional perovskite-based thin films have been reported widely, utilization of gain media with different dimensionalities, e.g. microwires and nanowires [349, 377], microplates [351, 358], and bulk single crystals [357], has also been explored. In particular, perovskite-based nanowires have received significant attention due to the naturally formed cavity effect that stems from having two end facets that are suitable for optical amplification [161, 349, 377]. In 2015, Zhu et al [377] were among the early researchers to demonstrate room-temperature, wavelength-tunable lasing in nanowires, with an average length of $\sim 20 \mu \mathrm{m}$, fabricated from compositionally tunable $\mathrm{CH}_{3} \mathrm{NH}_{3} \mathrm{PbBr}_{y} \mathrm{I}_{3-y}$ and $\mathrm{CH}_{3} \mathrm{NH}_{3} \mathrm{PbCl}_{x} \mathrm{Br}_{3-x}$. These devices covered the approximate wavelength range of $500-787 \mathrm{~nm}$. It is worth noting that the smooth rectangular end facets (see the inset of figure 25(a)) observed at both sides of the nanowire form Fabry-Pérot cavities necessary for optical amplification. Based on the observation of multiple, equally spaced lasing peaks, the Fabry-Pérot longitudinal cavity lasing modes are confirmed via the linear relationship between mode spacing $(\Delta \lambda)$ and the reciprocal of the nanowire length $\left(L^{-1}\right)$, as shown in figure $25(\mathrm{a})$. The $\Delta \lambda$ is calculated using equation (4) as shown below:

$$
\Delta \lambda=\frac{\lambda^{2}}{2 n_{\mathrm{g}} L}
$$

where $\lambda$ is the wavelength of guided light and $n_{\mathrm{g}}$ is the group refractive index.

The optical amplification effect from perovskite-based nanowires is dominated by their intrinsic Fabry-Pérot resonators. In contrast, in 2016, Zhang et al [349] reported that perovskite-based microplates rely on whispering gallery mode (WGM) resonators for light amplification. Lasing action from perovskite-based microplates (with equal side length varying from 3.1 to $14.1 \mu \mathrm{m}$ ) tends to localize on the four microplate edges (see inset of figure 25(b)) and the travelling wave is confined by total internal reflection within the boundary, whose characteristics resemble those of confined light in a WGM resonator. This is further evidenced by comparing the group refractive index $\left(n_{\mathrm{g}}\right)$ calculated from the WGM equation with other reported works. The WGM equation can be represented using equation (5), as shown below:

$$
\Delta \lambda=\frac{\lambda^{2}}{n_{\mathrm{g}} 2 \sqrt{2} d}
$$

where $\lambda$ is the wavelength of guided light and $d$ is the side length of a microplate. Figure 25(b) shows the fitted curve of (a)

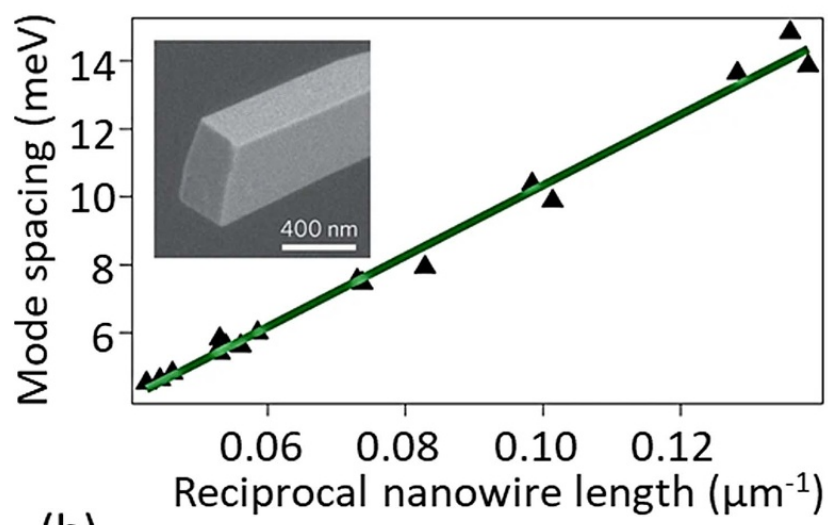

(b)

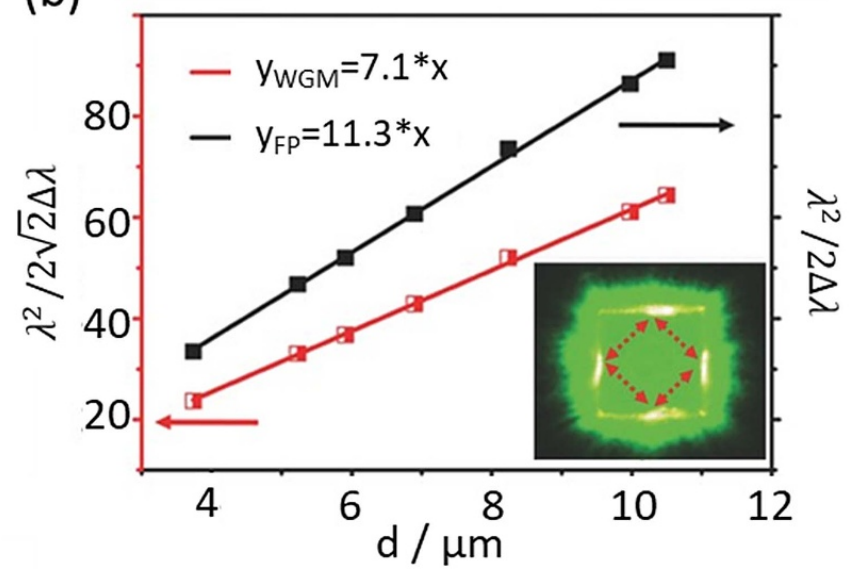

Figure 25. (a) Plot of mode spacing versus reciprocal of nanowire length based on the FP cavity formed by the two end facets. The inset shows the SEM image of the smooth end facet in the nanowire. Adapted by permission from Springer Nature Customer Service Centre GmbH: Springer Nature, Nature Materials [377], (C) 2015. (b) Plot of $\lambda^{2} / 2 \sqrt{2} \Delta \lambda$ versus the side length, $d$, of the microplate calculated based on WGM equation, as well as the plot of $\lambda^{2} / 2 \Delta \lambda$ versus $d$ calculated based on FP equation. The inset shows the lasing emission from the microplate with a side length of $6.5 \mu \mathrm{m}$ which is due to the WGM effect. [349] John Wiley \& Sons. Copyright (C) 2016 WILEY-VCH Verlag GmbH \& Co. KGaA, Weinheim.

$\lambda^{2} / 2 \sqrt{2} \Delta \lambda$ versus $d$ (red curve), which yields the $n_{\mathrm{g}}$ of 7.1 , closer to the value reported elsewhere. As compared to larger $n_{\mathrm{g}}$ of 11.3 that was determined based on the FP equation (see black curve in figure 25(b)), it is thus confirming that the lasing from the microplate is dominated by the WGMbased amplification effect. It is also noteworthy that singlemode lasing was achieved when the side length of the microplate was reduced to $3.1 \mu \mathrm{m}$ since the mode spacing $(5 \mathrm{~nm}$, in this case) was greater than the transition linewidth. Reduceddimensionality gain media are pivotal for enhanced quantum confinement, as well as for the development of nanophotonic devices and subsequent integration with existing photonic integrated circuits.

It is further envisaged that recent advances in ligand passivation may reduce defects in perovskite-based nanorods. For instance, Wang et al [343] recently reported a record-breaking 
net mode gain of up to $980 \mathrm{~cm}^{-1}$ in $\mathrm{CsPbBr}_{3}$ nanorodbased gain media with a diameter of $\sim 5.8 \mathrm{~nm}$ and a length of $\sim 26 \mathrm{~nm}$ using an intermediate monomer reservoir synthetic strategy. Similarly, group-III nitrides have benefitted from low-dimensionality nanostructures such as the previously discussed QDs [314, 378] and nanorods or nanowires [4, 327, 379].

3.2.5. Surface passivation. Perovskite-based material photostability has also improved substantially since early results where photoemission lasted for less than a few hours of continuous operation. For instance, a novel surface passivation technique demonstrated by Pan et al [155] in 2015, in which $\mathrm{CsPbBr}_{3}$-based QD surfaces were coated with an inorganic-organic hybrid ion pair, allowed passivated thin films to exhibit stable ASE operation for $\geqslant 34 \mathrm{~h}$ under ambient conditions. This demonstration laid a strong foundation for stable, heat- and moisture-resistant operation of perovskitebased gain media that is crucial to future applications.

Another passivation technique that uses a $\mathrm{CsPbBr} 3 / \mathrm{SiO}_{2}$ core/shell structure was demonstrated by $\mathrm{Hu}$ et al [157]. The researchers achieved recoverable ASE operation even after 2 months of storage under ambient conditions, as well as comparably lower threshold and more stable emission than that of $\mathrm{CsPbBr}_{3}$ QDs without a passivating shell. It was pointed out that the observed lower threshold and higher efficiency for ASE in core/shell structure could potentially be attributed to the large refractive index differences at the $\mathrm{SiO}_{2}$-air interface, which thus naturally forms a cavity that bounds the emitted light from the $\mathrm{CsPbBr}_{3}$ QDs back into the gain medium. In particular, the core/shell passivating method is also known to effectively suppress the PL blinking effect from colloidal $\mathrm{NCs}$, i.e. random switching between an emission state and dark state due to nonradiative Auger recombination via charge transfer. Such a passivation technique had also been commonly applied to other colloidal NCs, e.g. CdSe/CdS [380] and $\mathrm{CdTe} / \mathrm{ZnS}$ [381]. Importantly, the core/shell structure is also known to isolate the perovskite-based QDs from moist environments, as well as to increase susceptibility when used as the amplification medium and for subsequent fabrication steps without causing undesirable quenching in the PL. The use of perovskite-based QDs encompassing with a passivating shell structure would also be crucial in addressing the instability in terms of photoluminescence, preventing structural dissociation due to external moist environment, as well as for the realization of long-lasting lasing devices and applications. However, in addition to concerns about photostability, thermal issues in perovskite-based lasing devices remain largely unexplored and require significant attention, especially with respect to future electrically pumped lasing device designs. These devices are often required to operate at elevated temperatures. This may lead to malfunctions unless the design addresses heat dissipation.

Electrically injected perovskite-based lasers are still far from reaching high levels of technological readiness with respect to fabrication techniques and on-chip monolithic integration, as compared to group-III-nitride. However, the development of related materials is on a promising path because these materials benefit from lower capital costs than group-III-nitride materials. As shown previously, Br-based perovskite nanostructures have also been envisaged to partly address the 'green-yellow gap' in conventional group-IIInitride materials. Integration of group-III-nitride materials with perovskite-based gain media may allow the two technologies to further complement each other, for example via the enhanced quantum confinement effect, and thus completing a suite of technologies that covers the visible wavelength spectrum and supports various optoelectronic applications.

\section{Conclusions and perspectives}

As group-III-nitride laser and superluminescent devices have gained maturity and industrial adoption, halide-perovskite has emerged as another class of semiconductor with desired colortunable characteristics. In a regime where group-III nitrides fall short, metal-halide-perovskite semiconductors can potentially fill important gaps, especially for low-cost production. Solution processability in the green-yellow gap (530-600 nm) promises further lasing and ASE devices that may complement group-III nitride devices. While it is noted that the operating lives of halide perovskites have improved significantly from a few tens of hours to thousands of hours over the years and with the commercialization of perovskite-based solar cells expected from companies such as Oxford PV, Saule Technologies, and Tandem PV [382], development of more rigorous processing techniques, e.g. grating patterning, facet polishing, and doping, is required for electrically injected perovskite-based superluminescent and laser diodes. The main sources of concern are chemical sensitivity to common processing solvents, e.g. photoresists, strippers, and wet etchants [383], and subsequent photodegradation of perovskite-based structures. Compatibility of perovskite-based materials with industry-standard processing remains critical and represents a challenge. Moreover, the inclusion of lead in perovskite is regarded as a barrier to commercialization because of concerns over environmental pollution. Although companies such as Oxford PV and Saule Technologies have rebutted these claims and indicated that only a negligible amount of lead is included [382], some researchers are developing lead-free perovskites [384-386] that may potentially pave a new path towards more environmentally friendly optoelectronic devices. In addition to general application to perovskite-based LEDs, superluminescent diodes, and laser diodes for solid-state lighting, perovskite-based media remain attractive as conversion phosphors for high-speed visible light communication at both the transmitter and receiver ends [150, 387]. Furthermore, heterogeneous integration of both classes of semiconductor, i.e. group-III nitrides and perovskites, can offer new opportunities not previously available due to constraints related to high electrical injection density, lattice constants, crystal structures, and thermal expansion coefficient mismatches. Such integration may offer simultaneous optical feedback and external passivation, while the high thermal conductivities of group-IIInitride media ( $>230 \mathrm{~W} \mathrm{~m}^{-1} \mathrm{~K}$ ) may mitigate thermal issues associated with perovskite-based gain media. This is known to 
be important for improving device reliability [347]. However, as researchers pursue halide-perovskites and heterogeneous integration of group-III nitrides with halide-perovskites for lasing and ASE devices, standardized reporting of semiconductors and device characterization will be required to maintain scientific rigor [388] and address material stability [389] as the field gains popularity and moves towards technological maturity.

Current group-III nitride light emitting technology is moving towards new frontiers. Material-related developments are ongoing in the areas of polarization-assisted carrier concentration enhancement for ultraviolet emitters [76], tunnel junctions, and porous reflectors for blue vertical-emitting lasers [390, 391], and QDs and non- or semi-polar platforms for increased efficiency at green wavelengths [116, 314], including strain compensating engineering methods used to produce red-emitting InGaN-based devices [42]. While there is room for improvement in each of these segments, the device community has an interest to develop visible lasers into photonic integrated circuits [254, 392-394]. Heterogeneous integration of blue lasers with visible waveguides is an area of opportunity for topics of current interest such as beam steering for displays [395], optogenetics [396], and underwater applications where visible light exhibits the highest penetration [397]. Similarly, visible-light photonic integrated circuits can benefit from integrated DFB gratings, multiple section design including photodetectors, modulators, and amplifiers, and visiblelight waveguide development [398]. The potential integration of this platform with perovskites remains to be explored but could eventually accelerate the utilization of a broader range of wavelengths, which is needed for reconfigurable multifunctional photonics on a chip.

\section{Acknowledgments}

This work is based upon research supported in part by the U. S. Office of Naval Research under award number N6290919-1-2079 (KAUST reference number: RGC/3/4119-01-01) and in part by the King Abdullah University of Science and Technology (KAUST) Office of Sponsored Research (OSR) under Award No. OSR-CRG2017-3417, and KAUST baseline funding BAS/1/1614-01-01. T K N and B S O. acknowledge support from King Abdulaziz City for Science and Technology for the establishment of KACST-Technology-InnovationCenter on Solid State Lighting at KAUST (Grant No. KACST TIC R2-FP-008).

\section{ORCID iDs}

Tien Khee Ng (D) https://orcid.org/0000-0002-1480-6975 Jorge A Holguin-Lerma (D) https://orcid.org/0000-00030138-9073

Chun Hong Kang (D) https://orcid.org/0000-0003-4649-1127 Islam Ashry (D) https://orcid.org/0000-0002-6130-998X Huafan Zhang (D) https://orcid.org/0000-0002-8172-2632 Boon S Ooi (D) https://orcid.org/0000-0001-9606-5578

\section{References}

[1] Acharya A R 2015 Group III-nitride semiconductors: preeminent materials for modern electronic and optoelectronic applications Himalayan Phys. 5 22-26

[2] Hardy M T, Feezell D F, Denbaars S P and Nakamura S 2011 Group III-nitride lasers: a materials perspective Mater. Today 14 408-15

[3] Zhao S, Nguyen H P T, Kibria M G and Mi Z 2015 III-nitride nanowire optoelectronics Prog. Quantum Electron. 44 14-68

[4] Zhao C, Alfaraj N, Chandra Subedi R, Liang J W, Alatawi A A, Alhamoud A A, Ebaid M, Alias M S, Ng T K and Ooi B S 2018 III-nitride nanowires on unconventional substrates: from materials to optoelectronic device applications Prog. Quantum Electron. 61 1-31

[5] Meneghesso G, Meneghini M, Rossetto I, Bisi D, Stoffels S, Van Hove M, Decoutere S and Zanoni E 2016 Reliability and parasitic issues in GaN-based power HEMTs: a review Semicond. Sci. Technol. 31093004

[6] Millán J 2012 A review of WBG power semiconductor devices CAS 2012 (Int. Semiconductor Conf.) pp 57-66

[7] Li D, Jiang K, Sun X and Guo C 2018 AlGaN photonics: recent advances in materials and ultraviolet devices $A d v$. Opt. Photon. 10 43-110

[8] Chatterjee U, Park J H, Um D Y and Lee C R 2017 III-nitride nanowires for solar light harvesting: a review Renew. Sustain. Energy Rev. 79 1002-15

[9] Yadav S K, Wang J and Liu X Y 2016 Ab initio modeling of zincblende AlN layer in Al-AlN-TiN multilayers J. Appl. Phys. 119224304

[10] Ambacher O 1998 Growth and applications of group III-nitridese J. Phys. D: Appl. Phys. 312653

[11] Schubert E F 2003 Light-Emitting Diodes (Cambridge: Cambridge University Press)

[12] Chuang S L 2009 Physics of Photonic Devices (New York: Wiley)

[13] Wood C and Jena D 2008 Polarization Effects in Semiconductors: From Ab Initio Theory to Device Applications (New York: Springer)

[14] Park S H and Chuang S L 2000 Spontaneous polarization effects in wurtzite GaN/AlGaN quantum wells and comparison with experiment Appl. Phys. Lett. 76 1981-3

[15] Park S H 2003 Piezoelectric and spontaneous polarization effects on many-body optical gain of wurtzite $\mathrm{InGaN} / \mathrm{GaN}$ quantum well with arbitrary crystal orientation Japan. J. Appl. Phys. 142 5052-5

[16] Ambacher O et al 1999 Role of spontaneous and piezoelectric polarization induced effects in group-III nitride based heterostructures and devices Phys. Status Solidi 216 381-9

[17] Wu J, Walukiewicz W, Yu K M, Ager J W, Li S X, Haller E E, Lu H and Schaff W J 2003 Universal bandgap bowing in group-III nitride alloys Solid State Commun. 127 411-4

[18] Butcher K S A and Tansley T L 2005 InN, latest development and a review of the band-gap controversy Superlattices Microstruct. 38 1-37

[19] Tansley T L and Foley C P 1984 Electron mobility in indium nitride Electron. Lett. 20 1066-8

[20] Davydov V Y et al 2002 Absorption and emission of hexagonal InN. Evidence of narrow fundamental band gap Phys. Status Solidi 229 r1-3

[21] Higashiwaki M and Matsui T 2004 Estimation of band-gap energy of intrinsic InN from photoluminescence properties of undoped and Si-doped InN films grown by plasma-assisted molecular-beam epitaxy J. Cryst. Growth 269 162-6 
[22] Maruska H P and Tietjen J J 1969 The preparation and properties of vapor-deposited single-crystal-line $\mathrm{GaN}$ Appl. Phys. Lett. $15327-9$

[23] Yim W M, Stofko E J, Zanzucchi P J, Pankove J I, Ettenberg M and Gilbert S L 1973 Epitaxially grown AlN and its optical band gap J. Appl. Phys. 44 292-6

[24] Pankove J I, Miller E A, Richman D and Berkeyheiser J E 1971 Electroluminescence in GaN J. Lumin. 4 63-66

[25] Pankove J I, Miller E A and Berkeyheiser J E 1972 GaN blue light-emitting diodes J. Lumin. 5 84-86

[26] Maruska H P and Rhines W C 2015 A modern perspective on the history of semiconductor nitride blue light sources Solid State Electron. 111 32-41

[27] Usui A 1997 Bulk GaN crystal with low defect density grown by hydride vapor phase epitaxy Materials Research Society Symp.-Proc. vol 482 (MRS) pp 233-44

[28] Kim S T, Lee Y J, Moon D C, Hong C H and Yoo T K 1998 Preparation and properties of free-standing HVPE grown GaN substrates J. Cryst. Growth 194 37-42

[29] Naniwae K, Itoh S, Amano H, Itoh K, Hiramatsu K and Akasaki I 1990 Growth of single crystal GaN substrate using hydride vapor phase epitaxy J. Cryst. Growth $99381-4$

[30] Amano H, Sawaki N, Akasaki I and Toyoda Y 1986 Metalorganic vapor phase epitaxial growth of a high quality GaN film using an AlN buffer layer Appl. Phys. Lett. 48 353-5

[31] Nakamura S, Mukai T and Senoh M 1994 Candela-class high-brightness InGaN/AlGaN double-heterostructure blue-light-emitting diodes Appl. Phys. Lett. 64 1687-9

[32] Su J et al 2005 Catalytic growth of group III-nitride nanowires and nanostructures by metalorganic chemical vapor deposition Appl. Phys. Lett. 86013105

[33] Wang J, Nozaki M, Ishikawa Y, Hao M S, Morishima Y, Wang T, Naoi Y and Sakai S 1999 Fabrication of nanoscale structures of InGaN by MOCVD lateral overgrowth J. Cryst. Growth 197 48-53

[34] Kuykendall T, Pauzauskie P J, Zhang Y, Goldberger J, Sirbuly D, Denlinger J and Yang P 2004 Crystallographic alignment of high-density gallium nitride nanowire arrays Nat. Mater. 3 524-8

[35] Wierer J J, Tansu N, Fischer A J and Tsao J Y 2016 III-nitride quantum dots for ultra-efficient solid-state lighting Laser Photon. Rev. 10 612-22

[36] Sánchez-García M A, Pau J L, Naranjo F, Jiménez A, Fernández S, Ristic J, Calle F, Calleja E and Muñoz E 2002 Plasma-assisted MBE growth of group-III nitrides: from basics to device applications Mater. Sci. Eng. B 93 189-96

[37] Ohkawa K, Watanabe T, Sakamoto M, Hirako A and Deura M 2012 740-nm emission from InGaN-based LEDs on $c$-plane sapphire substrates by MOVPE J. Cryst. Growth 343 13-16

[38] Zou X, Wong K M, Zhu X, Chong W C, Ma J and Lau K M 2013 High-performance green and yellow LEDs grown on $\mathrm{SiO}_{2}$ nanorod patterned $\mathrm{GaN} / \mathrm{Si}$ templates IEEE Electron Device Lett. 34 903-5

[39] Zhang J, Xiong C, Liu J, Quan Z, Wang L and Jiang F 2014 High brightness InGaN-based yellow light-emitting diodes with strain modulation layers grown on Si substrate Appl. Phys. A 114 1049-53

[40] Conroy M, Li H, Kusch G, Zhao C, Ooi B, Edwards P R, Martin R W, Holmes J D and Parbrook P J 2016 Site controlled red-yellow-green light emitting InGaN quantum discs on nano-tipped GaN rods Nanoscale 8 11019-26

[41] Iida D, Niwa K, Kamiyama S and Ohkawa K 2016 Demonstration of InGaN-based orange LEDs with hybrid multiple-quantum-wells structure Appl. Phys. Express 9111003
[42] Iida D, Zhuang Z, Kirilenko P, Velazquez-Rizo M and Ohkawa K 2020 Demonstration of low forward voltage InGaN-based red LEDs Appl. Phys. Express 13031001

[43] Malin T, Mansurov V, Galitsyn Y and Zhuravlev K 2014 Thermodynamic and kinetic aspects of AlN crystal formation on (0001) $\mathrm{Al}_{2} \mathrm{O}_{3}$ surface by ammonia MBE Phys. Status Solidi c 11 613-6

[44] Oliver R A, Kappers M J, Humphreys C J and Briggs G A D 2004 The influence of ammonia on the growth mode in InGaN/GaN heteroepitaxy J. Cryst. Growth 272 393-9

[45] Ravikiran L, Agrawal M, Dharmarasu N and Radhakrishnan K 2013 Effect of stress mitigating layers on the structural properties of $\mathrm{GaN}$ grown by ammonia molecular beam epitaxy on $100 \mathrm{~mm} \mathrm{Si(111)} \mathrm{Japan.} \mathrm{J.}$ Appl. Phys. 52 08JE05

[46] Dharmarasu N, Radhakrishnan K, Sun Z Z and Agrawal M 2011 Realization of two-dimensional electron gas in $\mathrm{AlGaN} / \mathrm{GaN}$ HEMT structure grown on $\mathrm{Si}(111)$ by PA-MBE Phys. Status Solidi c 8 2075-7

[47] Damilano B, Grandjean N, Dalmasso S and Massies J 1999 Room-temperature blue-green emission from $\mathrm{InGan} / \mathrm{GaN}$ quantum dots made by strain-induced islanding growth Appl. Phys. Lett. 75 3751-3

[48] Grandjean N, Damilano B and Massies J 2001 Group-III nitride quantum heterostructures grown by molecular beam epitaxy J. Phys.: Condens. Matter 136945

[49] $\mathrm{Ng} \mathrm{T} \mathrm{K}$ et al 2014 Red to near-infrared emission from InGaN/GaN quantum-disks-in-nanowires LED Conf. on Lasers and Electro-Optics Europe-Technical Digest (January) vol 2014 (Optical Society of America) p SM2J.2

[50] Mastro M A, Holm R T, Bassim N D, Eddy C R, Gaskill D K, Henry R L and Twigg M E 2005 High-reflectance III-nitride distributed Bragg reflectors grown on Si substrates Appl. Phys. Lett. 87 1-3

[51] Prabaswara A, Birch J, Junaid M, Serban E A, Hultman L and Hsiao C-L 2020 Review of GaN thin film and nanorod growth using magnetron sputter epitaxy Appl. Sci. 103050

[52] Banerjee S, Aarnink A A I, Gravesteijn D J and Kovalgin A Y 2019 Thermal atomic layer deposition of polycrystalline gallium nitride J. Phys. Chem. C $12323214-25$

[53] Vispute R D, Talyansky V, Sharma R P, Choopun S, Downes M, Venkatesan T, Jones K A, Iliadis A A, Asif Khan M and Yang J W 1997 Growth of epitaxial GaN films by pulsed laser deposition Appl. Phys. Lett. 71 102-4

[54] Biju K P, Subrahmanyam A and Jain M K 2009 Low-temperature growth of polycrystalline GaN films using modified activated reactive evaporation $J$. Cryst. Growth 311 2275-80

[55] Kang J, Mitsuhashi T, Kuroda K and Okido M 2019 Low-temperature synthesis of GaN film from aqueous solution by electrodeposition J. Appl. Electrochem. 49 871-81

[56] Chen R, Zhou W and Hoi Sing K 2012 Top-gate thin-film transistors based on GaN channel layer Appl. Phys. Lett. 100022111

[57] Jeong J et al 2020 Remote heteroepitaxy of GaN microrod heterostructures for deformable light-emitting diodes and wafer recycle $S c i$. Adv. 6 eaaz5180

[58] Min J-W et al 2018 Unleashing the potential of molecular beam epitaxy grown AlGaN-based ultraviolet-spectrum nanowires devices J. Nanophoton. 1243511

[59] Morkoç H 2001 III-nitride semiconductor growth by MBE: recent issues J. Mater. Sci. Mater. Electron. 12 677-95

[60] Kimura T, Horibuchi K, Kataoka K and Nakamura D 2018 Macro-defect-free homoepitaxial GaN growth through halogen-free vapor-phase epitaxy on native GaN seeds $J$. Cryst. Growth 494 17-25 
[61] Dadgar A 2019 GaN-On-Si epitaxy Encyclopedia of Applied Physics (New York: Wiley) pp 1-13

[62] Shin H, Jeon K, Jang Y, Gang M, Choi M, Park W and Park K 2013 Comparison of the microstructural characterizations of GaN layers grown on $\mathrm{Si}(111)$ and on sapphire J. Korean Phys. Soc. 63 1621-4

[63] Kaminski N and Hilt O 2014 SiC and GaN devices-wide bandgap is not all the same IET Circuits, Devices Syst. $8227-36$

[64] Panalytical M 2018 (Booklet) XRD of Gallium Nitride and Related Compounds: Strain, Composition and Layer Thickness

[65] Zhang L, Hong Lee K, Riko I M, Huang C C, Kadir A, Lee K E, Jin Chua S and Fitzgerald E A 2017 MOCVD growth of GaN on SEMI-spec $200 \mathrm{~mm} \mathrm{Si} \mathrm{Semicond.} \mathrm{Sci.}$ Technol. 32065001

[66] Waldrip K E, Han J, Figiel J J, Zhou H, Makarona E and Nurmikko A V 2001 Stress engineering during metalorganic chemical vapor deposition of $\mathrm{AlGaN} / \mathrm{GaN}$ distributed Bragg reflectors Appl. Phys. Lett. 78 3205-7

[67] Liu Y S et al 2016 Strain management of AlGaN-based distributed Bragg reflectors with $\mathrm{GaN}$ interlayer grown by metalorganic chemical vapor deposition Appl. Phys. Lett. 109081103

[68] Kim M H, Schubert M F, Dai Q, Kim J K, Schubert E F, Piprek J and Park Y 2007 Origin of efficiency droop in GaN-based light-emitting diodes Appl. Phys. Lett. 91183507

[69] Piprek J 2010 Efficiency droop in nitride-based light-emitting diodes Phys. Status Solidi a 207 2217-25

[70] Nakamura S, Mukai T and Senoh M 1991 High-power gan p-n junction blue-light-emitting diodes Japan. J. Appl. Phys. 30 L1998-2001

[71] Nakamura S, Pearton S and Fasol G 2000 The Blue Laser Diode (Berlin Heidelberg: Springer-Verlag)

[72] Yan Y, Li J, Wei S H and Al-Jassim M M 2007 Possible approach to overcome the doping asymmetry in wideband gap semiconductors Phys. Rev. Lett. 98135506

[73] Kozodoy P, Hansen M, DenBaars S P and Mishra U K 1999 Enhanced $\mathrm{Mg}$ doping efficiency in $\mathrm{Al}_{0.2} \mathrm{Ga}_{0.8} \mathrm{~N} / \mathrm{GaN}$ superlattices Appl. Phys. Lett. 74 3681-3

[74] Nam K B, Nakarmi M L, Li J, Lin J Y and Jiang H X 2003 $\mathrm{Mg}$ acceptor level in AlN probed by deep ultraviolet photoluminescence Appl. Phys. Lett. 83 878-80

[75] Takano T, Mino T, Sakai J, Noguchi N, Tsubaki K and Hirayama H 2017 Deep-ultraviolet light-emitting diodes with external quantum efficiency higher than $20 \%$ at 275 $\mathrm{nm}$ achieved by improving light-extraction efficiency Appl. Phys. Express 10031002

[76] Zhang Z, Kushimoto M, Sakai T, Sugiyama N, Schowalter L J, Sasaoka C and Amano H 2019 A 271.8 $\mathrm{nm}$ deep-ultraviolet laser diode for room temperature operation Appl. Phys. Express 12124003

[77] Nam K B, Li J, Nakarmi M L, Lin J Y and Jiang H X 2004 Unique optical properties of $\mathrm{AlGaN}$ alloys and related ultraviolet emitters Appl. Phys. Lett. 84 5264-6

[78] Detchprohm T, Li X, Shen S C, Yoder P D and Dupuis R D 2017 III-N wide bandgap deep-ultraviolet lasers and photodetectors Semiconductors and Semimetals vol 96, ed Z Mi and C Jagadish (Amsterdam: Elsevier) pp 121-66

[79] Suzuki M, Uenoyama T and Yanase A 1995 First-principles calculations of effective-mass parameters of AlN and GaN Phys. Rev. B 52 8132-9

[80] Guttmann M, Mehnke F, Belde B, Wolf F, Reich C, Sulmoni L, Wernicke T and Kneissl M 2019 Optical light polarization and light extraction efficiency of AlGaN-based LEDs emitting between 264 and $220 \mathrm{~nm}$ Japan. J. Appl. Phys. 58 SCCB20
[81] Kneissl M and Rass J 2016 III-Nitride Ultraviolet Emitters (Berlin: Springer)

[82] Piprek J 2007 Nitride Semiconductor Devices: Principles and Simulation (New York: Wiley)

[83] Morkoç H 2009 Optical processes in semiconductors and optical properties of nitride semiconductors and heterostructures Handbook of Nitride Semiconductors and Devices (New York: Wiley) pp 491-827

[84] Zhang R and Xiu X 2019 GaN substrate material for III-V semiconductor epitaxy growth Light-Emitting Diodes (Berlin: Springer) pp 1-39

[85] Jmerik V N, Nechaev D V and Ivanov S V 2018 Kinetics of metal-rich PA molecular beam epitaxy of AlGaN heterostructures for mid-UV photonics Molecular Beam Epitaxy (Amsterdam: Elsevier) pp 135-79

[86] Yan J, Wang J, Liu Y and Li J 2019 Al-rich III-nitride materials and ultraviolet light-emitting diodes Light-Emitting Diodes (Berlin: Springer) pp 245-79

[87] Arif R A, Ee Y K and Tansu N 2007 Polarization engineering via staggered $\mathrm{InGaN}$ quantum wells for radiative efficiency enhancement of light emitting diodes Appl. Phys. Lett. 91091110

[88] Li H, Li P, Kang J, Li Z, Zhang Y, Li Z, Li J, Yi X, Li J and Wang G 2013 Quantum efficiency enhancement of 530nm InGaN green light-emitting diodes with shallow quantum well Appl. Phys. Express 6052102

[89] Wu F, Sun H, AJia I A, Roqan I S, Zhang D, Dai J, Chen C, Feng Z C and Li X 2017 Significant internal quantum efficiency enhancement of GaN/AlGaN multiple quantum wells emitting at $\sim 350 \mathrm{~nm}$ via step quantum well structure design J. Phys. D: Appl. Phys. 50245101

[90] Mathew C S, Kwang-Choong K, Robert M F, Daniel F F, Daniel A C, Makoto S, Kenji F, James S S, Steven P D and Shuji N 2007 Demonstration of nonpolar m-plane InGaN/GaN laser diodes Japan. J. Appl. Phys. 46 L190

[91] Wang T 2016 Topical review: development of overgrown semi-polar GaN for high efficiency green/yellow emission Semicond. Sci. Technol. 31093003

[92] Kang C H et al 2019 Semipolar (20-2-1) InGaN/GaN micro-photodetector for gigabit-per-second visible light communication Appl. Phys. Express 13014001

[93] Zhao Y, Tanaka S, Pan C-C, Fujito K, Feezell D, Speck J S, DenBaars S P and Nakamura S 2011 High-power blue-violet semipolar (20-2-1) InGaN/GaN light-emitting diodes with low efficiency droop at $200 \mathrm{~A} \mathrm{~cm}^{-2} \mathrm{Appl}$. Phys. Express 4082104

[94] Speck J S and Chichibu S F 2009 Nonpolar and semipolar group III nitride-based materials MRS Bull. 34 304-12

[95] Zhao Y et al 2012 Indium incorporation and emission properties of nonpolar and semipolar InGaN quantum wells Appl. Phys. Lett. 100201108

[96] Simon J, Protasenko V, Lian C, Xing H and Jena D 2010 Polarization-induced hole doping in wide-band-gap uniaxial semiconductor heterostructures Science 327 60-64

[97] Li S, Zhang T, Wu J, Yang Y, Wang Z, Wu Z, Chen Z and Jiang Y 2013 Polarization induced hole doping in graded AlxGa $1-x \mathrm{~N}(x=0.7 \sim 1)$ layer grown by molecular beam epitaxy Appl. Phys. Lett. 102062108

[98] Janjua B, Ng T K, Alyamani A Y, El-Desouki M M and Ooi B S 2014 Enhancing carrier injection using graded superlattice electron blocking layer for UVB light-emitting diodes IEEE Photon. J 61600312

[99] Jena D et al 2002 Realization of wide electron slabs by polarization bulk doping in graded III-V nitride semiconductor alloys Appl. Phys. Lett. 81 4395-7

[100] Wei J, Ouyang D F, Deng S Y, Peng F, Yang C and Luo X R 2019 Polarization doping technology towards high performance GaN-based heterostructure 
devices-IOPscience IOP Conf. Series: Materials Science and Engineering (Sanya, China) p 012052

[101] So B, Kim J, Kwak T, Kim T, Lee J, Choi U and Nam O 2018 Improved carrier injection of $\mathrm{AlGaN}$-based deep ultraviolet light emitting diodes with graded superlattice electron blocking layers $R S C A d v .835528-33$

[102] Janjua B et al 2018 Ultraviolet-A LED based on quantum-disks-in-algan-nanowires-optimization and device reliability IEEE Photon. J 102200711

[103] Si Q, Chen H, Li S, Lu S and Kang J 2017 Improved characteristics of $\mathrm{AlGaN}$-based deep ultraviolet light-emitting diodes with superlattice p-type doping IEEE Photon. J 92200807

[104] Ren Z, Yu H, Liu Z, Wang D, Xing C, Zhang H, Huang C, Long $S$ and Sun $\mathrm{H} 2020$ Band engineering of III-nitride-based deep-ultraviolet light-emitting diodes: a review J. Appl. Phys. 53073002

[105] Saito S, Hashimoto R, Hwang J and Nunoue S 2013 InGaN light-emitting diodes on c-face sapphire substrates in green gap spectral range Appl. Phys. Express 6111004

[106] Du C, Ma Z, Zhou J, Lu T, Jiang Y, Zuo P, Jia H and Chen H 2014 Enhancing the quantum efficiency of InGaN yellow-green light-emitting diodes by growth interruption Appl. Phys. Lett. 105071108

[107] Narukawa Y, Ichikawa M, Sanga D, Sano M and Mukai T 2010 White light emitting diodes with super-high luminous efficacy J. Phys. D: Appl. Phys. $\mathbf{4 3} 354002$

[108] Ajay A, Kotsar Y and Monroy E 2018 Infrared emitters using III-nitride semiconductors Nitride Semiconductor Light-Emitting Diodes (LEDs): Materials, Technologies, and Applications 2nd edn (Amsterdam: Elsevier) pp 587-617

[109] Zhu D and Humphreys C J 2016 Solid-state lighting based on light emitting diode technology Optics in Our Time (Berlin: Springer) pp 87-118

[110] Broell M, Sundgren P, Rudolph A, Schmid W, Vogl A and Behringer M 2014 New developments on high-efficiency infrared and InGaAlP light-emitting diodes at OSRAM opto semiconductors Proc. SPIE 9003 90030L

[111] Auf Der Maur M, Pecchia A, Penazzi G, Rodrigues W and Di Carlo A 2016 Efficiency drop in green InGaN/GaN light emitting diodes: the role of random alloy fluctuations Phys. Rev. Lett. 116027401

[112] Li P P et al 2018 Very high external quantum efficiency and wall-plug efficiency $527 \mathrm{~nm}$ InGaN green LEDs by MOCVD Opt. Express 2633108

[113] Jiang F et al 2019 Efficient InGaN-based yellow-light-emitting diodes Photon. Res. 7144

[114] Liu M, Zhao J, Zhou S, Gao Y, Hu J, Liu X and Ding X 2018 An InGaN/GaN superlattice to enhance the performance of green LEDs: exploring the role of V-pits Nanomaterials 8450

[115] Zhou S, Liu X, Yan H, Gao Y, Xu H, Zhao J, Quan Z, Gui C and Liu S 2018 The effect of nanometre-scale V-pits on electronic and optical properties and efficiency droop of GaN-based green light-emitting diodes Sci. Rep. 811053

[116] Murayama M, Nakayama Y, Yamazaki K, Hoshina Y, Watanabe H, Fuutagawa N, Kawanishi H, Uemura T and Narui H 2018 Watt-class green $(530 \mathrm{~nm})$ and blue (465 nm) laser diodes Phys. Status Solidi 2151700513

[117] Nakatsu Y, Nagao Y, Hirao T, Hara Y, Masui S, Yanamoto T and Nagahama S 2020 Blue and green InGaN semiconductor lasers as light sources for displays Proc. SPIE $11280112800 \mathrm{~S}$

[118] Strauß U, Avramescu A, Lermer T, Queren D, Gomez-Iglesias A, Eichler C, Müller J, Brüderl G and Lutgen S 2011 Pros and cons of green InGaN laser on c-plane GaN Phys. Status Solidi b 248 652-7
[119] Rose G 1839 Beschreibung einiger neuen Mineralien des Urals Ann. Phys. Chem. 124 551-73

[120] Wells H L 1893 Über die Cäsium- und Kalium-Bleihalogenide Z. Anorg. Chem. 3 195-210

[121] Goldschmidt V M 1926 Die Gesetze der Krystallochemie Naturwissenschaften 14 477-85

[122] Megaw H D 1945 Crystal structure of barium titanate Nature 155 484-5

[123] Weber D 1978 CH3NH3PbX3, ein Pb(II)-System mit kubischer Perowskitstruktur Z. Naturforsch. B 33b 1443-5

[124] Weber D 1978 CH3NH3SnBrxI3-x (x = 0-3), ein Sn(II)-System mit kubischer Perowskitstruktur $Z$. Naturforsch. B 33 862-5

[125] Kojima A, Teshima K, Shirai Y and Miyasaka T 2009 Organometal halide perovskites as visible-light sensitizers for photovoltaic cells J. Am. Chem. Soc. 131 6050-1

[126] Liu Y, Yang Z and Liu S F 2018 Recent progress in single-crystalline perovskite research including crystal preparation, property evaluation, and applications $A d v$. Sci. 51700471

[127] Zhou Y and Zhao Y 2019 Chemical stability and instability of inorganic halide perovskites Energy Environ. Sci. 12 1495-511

[128] Fu Y, Zhu H, Chen J, Hautzinger M P, Zhu X Y and Jin S 2019 Metal halide perovskite nanostructures for optoelectronic applications and the study of physical properties Nat. Rev. Mater. 4 169-88

[129] Wu H and Zhu X 2016 Perovskite oxide nanocrystals - synthesis, characterization, functionalization, and novel applications Perovskite Materials-Synthesis, Characterisation, Properties, and Applications (Rijeka: InTech) 153-83

[130] Ono L K and Qi Y 2018 Research progress on organic-inorganic halide perovskite materials and solar cells J. Phys. D: Appl. Phys. 51093001

[131] Saliba M, Correa-Baena J-P, Grätzel M, Hagfeldt A and Abate A 2018 Perovskite solar cells: from the atomic level to film quality and device performance Angew. Chem., Int. Ed. 57 2554-69

[132] Li Y F, Feng J and Sun H B 2019 Perovskite quantum dots for light-emitting devices Nanoscale 11 19119-39

[133] Akkerman Q A, D'Innocenzo V, Accornero S, Scarpellini A, Petrozza A, Prato M and Manna L 2015 Tuning the optical properties of cesium lead halide perovskite nanocrystals by anion exchange reactions J. Am. Chem. Soc. 137 10276-81

[134] Kitazawa N, Watanabe Y and Nakamura Y 2002 Optical properties of $\mathrm{CH}_{3} \mathrm{NH}_{3} \mathrm{PbX}_{3}(\mathrm{X}=$ halogen) and their mixed-halide crystals J. Mater. Sci. 37 3585-7

[135] Pourdavoud N et al 2018 Distributed feedback lasers based on $\mathrm{MAPbBr}_{3}$ Adv. Mater. Technol. 31700253

[136] Pourdavoud N et al 2019 Room-temperature stimulated emission and lasing in recrystallized cesium lead bromide perovskite thin films Adv. Mater. 311903717

[137] Hong K, Van Le Q, Kim S Y and Jang H W 2018 Low-dimensional halide perovskites: review and issues $J$. Mater. Chem. C 6 2189-209

[138] Gu L et al 2016 3D arrays of 1024-pixel image sensors based on lead halide perovskite nanowires Adv. Mater. 28 9713-21

[139] Chen J, Zhou S, Jin S, Li H and Zhai T 2015 Crystal organometal halide perovskites with promising optoelectronic applications J. Mater. Chem. C 4 11-27

[140] Lin H, Zhou C, Tian Y, Siegrist T and Ma B 2018 Low-dimensional organometal halide perovskites ACS Energy Lett. 3 54-62

[141] Saidaminov M I, Mohammed O F and Bakr O M 2017 Low-dimensional-networked metal halide perovskites: the next big thing ACS Energy Lett. 2 889-96 
[142] Zhang Y, Liu J, Wang Z, Xue Y, Ou Q, Polavarapu L, Zheng J, Qi X and Bao Q 2016 Synthesis, properties, and optical applications of low-dimensional perovskites Chem. Commun. 52 13637-55

[143] Xiang W and Tress W 2019 Review on recent progress of all-inorganic metal halide perovskites and solar cells $A d v$. Mater. 311902851

[144] Jena A K, Kulkarni A and Miyasaka T 2019 Halide perovskite photovoltaics: background, status, and future prospects Chem. Rev. 119 3036-103

[145] Duan J, Xu H, Sha W E I, Zhao Y, Wang Y, Yang X and Tang Q 2019 Inorganic perovskite solar cells: an emerging member of the photovoltaic community J. Mater. Chem. A $721036-68$

[146] Wei H and Huang J 2019 Halide lead perovskites for ionizing radiation detection Nat. Commun. 101066

[147] Zhao Y, Li C and Shen L 2018 Recent research process on perovskite photodetectors: a review for photodetector-materials, physics, and applications Chin. Phys. B 27127806

[148] Miao J and Zhang F 2019 Recent progress on highly sensitive perovskite photodetectors J. Mater. Chem. C 7 1741-91

[149] Wang Y, Gao M L, Wu J L and Zhang X W 2019 Metal halide perovskite photodetectors: material features and device engineering Chin. Phys. B 28018502

[150] Kang C H et al 2019 High-speed colour-converting photodetector with all-inorganic $\mathrm{Cs} \mathrm{PbBr}_{3}$ perovskite nanocrystals for ultraviolet light communication Light Sci. Appl. 894

[151] Van Le Q, Jang H W and Kim S Y 2018 Recent advances toward high-efficiency halide perovskite light-emitting diodes: review and perspective Small Methods 21700419

[152] Kumawat N K, Liu X K, Kabra D and Gao F 2019 Blue perovskite light-emitting diodes: progress, challenges and future directions Nanoscale 11 2109-20

[153] Stylianakis M, Maksudov T, Panagiotopoulos A, Kakavelakis G and Petridis K 2019 Inorganic and hybrid perovskite based laser devices: a review Materials 12859

[154] Lee K J et al 2019 Perovskite-based artificial multiple quantum wells Nano Lett. 19 3535-42

[155] Pan J et al 2015 Air-stable surface-passivated perovskite quantum dots for ultra-robust, single- and two-photon-induced amplified spontaneous emission J. Phys. Chem. Lett. 6 5027-33

[156] Veldhuis S A, Tay Y K E, Bruno A, Dintakurti S S H, Bhaumik S, Muduli S K, Li M, Mathews N, Sum T C and Mhaisalkar S G 2017 Benzyl alcohol-treated $\mathrm{CH}_{3} \mathrm{NH}_{3}$ $\mathrm{PbBr}_{3}$ nanocrystals exhibiting high luminescence, stability, and ultralow amplified spontaneous emission thresholds Nano Lett. 17 7424-32

[157] Hu Z et al 2018 Enhanced two-photon-pumped emission from in situ synthesized nonblinking $\mathrm{CsPbBr}{ }_{3} / \mathrm{SiO}_{2}$ nanocrystals with excellent stability Adv. Opt. Mater. 61700997

[158] Kim J, Ho-Baillie A and Huang S 2019 Review of novel passivation techniques for efficient and stable perovskite solar cells Sol. RRL 31800302

[159] Li J, Si J, Gan L, Liu Y, Ye Z and He H 2016 Simple approach to improving the amplified spontaneous emission properties of perovskite films ACS Appl. Mater. Interfaces 8 32978-83

[160] Castro-Hermosa S, Yadav S K, Vesce L, Guidobaldi A, Reale A, Di Carlo A and Brown T M 2017 Stability issues pertaining large area perovskite and dye-sensitized solar cells and modules J. Phys. D: Appl. Phys. 50033001

[161] Fu Y, Zhu H, Stoumpos C C, Ding Q, Wang J, Kanatzidis M G, Zhu X and Jin S 2016 Broad wavelength tunable robust lasing from single-crystal nanowires of cesium lead halide perovskites $(\mathrm{CsPbX} 3, \mathrm{X}=\mathrm{Cl}, \mathrm{Br}, \mathrm{I})$ ACS Nano $107963-72$

[162] Eperon G E, Stranks S D, Menelaou C, Johnston M B, Herz L M and Snaith H J 2014 Formamidinium lead trihalide: a broadly tunable perovskite for efficient planar heterojunction solar cells Energy Environ. Sci. 7 982-8

[163] Travis W, Glover E N K, Bronstein H, Scanlon D O and Palgrave R G 2016 On the application of the tolerance factor to inorganic and hybrid halide perovskites: a revised system Chem. Sci. 7 4548-56

[164] Cao W et al 2017 Halide-rich synthesized cesium lead bromide perovskite nanocrystals for light-emitting diodes with improved performance Chem. Mater. 29 5168-73

[165] Cheng L-P et al 2019 Efficient $\mathrm{CsPbBr}_{3}$ perovskite light-emitting diodes enabled by synergetic morphology control Adv. Opt. Mater. 71801534

[166] Cai W, Chen Z, Chen D, Su S, Xu Q, Yip H L and Cao Y 2019 High-performance and stable $\mathrm{CsPBr}_{3}$ light-emitting diodes based on polymer additive treatment RSC Adv. 9 27684-91

[167] Wang Y, Li X, Song J, Xiao L, Zeng H and Sun H 2015 All-inorganic colloidal perovskite quantum dots: a new class of lasing materials with favorable characteristics $A d v$. Mater. 27 7101-8

[168] Yakunin S, Protesescu L, Krieg F, Bodnarchuk M I, Nedelcu G, Humer M, De Luca G, Fiebig M, Heiss W and Kovalenko M V 2015 Low-threshold amplified spontaneous emission and lasing from colloidal nanocrystals of caesium lead halide perovskites Nat. Commun. 68056

[169] NREL Best research-cell efficiencies (available at: www.nrel.gov/pv/assets/pdfs/best-research-cellefficiencies.20191106.pdf)

[170] Green M A, Dunlop E D, Levi D H, Hohl-Ebinger J, Yoshita M and Ho-Baillie A W Y 2019 Solar cell efficiency tables (version 54) Prog. Photovolt. Res. Appl. 27 565-75

[171] Zhang Q et al 2019 Efficient metal halide perovskite light-emitting diodes with significantly improved light extraction on nanophotonic substrates Nat. Commun. 10727

[172] Lin K et al 2018 Perovskite light-emitting diodes with external quantum efficiency exceeding 20 per cent Nature 562 245-8

[173] Kang C et al 2019 Highly efficient and stable white light-emitting diodes using perovskite quantum dot paper Adv. Sci. 61902230

[174] Chang C Y, Solodukhin A N, Liao S Y, Mahesh K P O, Hsu C L, Ponomarenko S A, Luponosov Y N and Chao Y C 2019 Perovskite white light-emitting diodes based on a molecular blend perovskite emissive layer J. Mater. Chem. C 7 8634-42

[175] Lin C-H et al 2019 Hybrid-type white LEDs based on inorganic halide perovskite QDs: candidates for wide color gamut display backlights Photon. Res. 7579

[176] Mao J, Lin H, Ye F, Qin M, Burkhartsmeyer J M, Zhang H, Lu X, Wong K S and Choy W C H 2018 All-perovskite emission architecture for white light-emitting diodes ACS Nano 12 10486-92

[177] Ren Z, Xiao X, Ma R, Lin H, Wang K, Sun X W and Choy W C H 2019 Hole transport bilayer structure for quasi-2D perovskite based blue light-emitting diodes with high brightness and good spectral stability Adv. Funct. Mater. 291905339

[178] Georgiadou D G, Lin Y, Lim J, Ratnasingham S, McLachlan M A, Snaith H J and Anthopoulos T D 2019 High responsivity and response speed single-layer mixed-cation lead mixed-halide perovskite photodetectors 
based on nanogap electrodes manufactured on large-area rigid and flexible substrates Adv. Funct. Mater. 291901371

[179] Kang D-H, Pae S R, Shim J, Yoo G, Jeon J, Leem J W, Yu J S, Lee S, Shin B and Park J-H 2016 An ultrahigh-performance photodetector based on a perovskite-transition-metal-dichalcogenide hybrid structure Adv. Mater. 28 7799-806

[180] Deng W, Huang L, Xu X, Zhang X, Jin X, Lee S T and Jie J 2017 Ultrahigh-responsivity photodetectors from perovskite nanowire arrays for sequentially tunable spectral measurement Nano Lett. 17 2482-9

[181] Asuo I M, Fourmont P, Ka I, Gedamu D, Bouzidi S, Pignolet A, Nechache R and Cloutier S G 2019 Highly efficient and ultrasensitive large-area flexible photodetector based on perovskite nanowires Small 151804150

[182] Deng W et al 2019 All-sprayed-processable, large-area, and flexible perovskite/MXene-based photodetector arrays for photocommunication Adv. Opt. Mater. 71801521

[183] Tong S et al 2019 Fully-printed, flexible cesium-doped triple cation perovskite photodetector Appl. Mater. Today 15 389-97

[184] Liu Z, Li H, Qin C, Zhang T, Gu Y, Chen H, Zheng H and Li S 2019 Solution-processed inorganic perovskite flexible photodetectors with high performance Nanoscale Res. Lett. 14284

[185] Sutherland B R, Johnston A K, Ip A H, Xu J, Adinolfi V, Kanjanaboos P and Sargent E H 2015 Sensitive, fast, and stable perovskite photodetectors exploiting interface engineering ACS Photon. 2 1117-23

[186] Kang C H et al 2019 High-speed ultraviolet-C photodetector based on frequency down-converting $\mathrm{Cs} \mathrm{PbBr}_{3}$ perovskite nanocrystals on silicon platform 2019 IEEE Photonics Conf. (IEEE)

[187] Yang Y, Dai H, Yang F, Zhang Y, Luo D, Zhang X, Wang K, Sun X W and Yao J 2019 All-perovskite photodetector with fast response Nanoscale Res. Lett. 14291

[188] Wang H and Kim D H 2017 Perovskite-based photodetectors: materials and devices Chem. Soc. Rev. 46 5204-36

[189] Nedelcu G, Protesescu L, Yakunin S, Bodnarchuk M I, Grotevent M J and Kovalenko M V 2015 Fast anion-exchange in highly luminescent nanocrystals of cesium lead halide perovskites $(\mathrm{CsPbX} 3, \mathrm{X}=\mathrm{Cl}, \mathrm{Br}, \mathrm{I})$ Nano Lett. 15 5635-40

[190] Sutherland B R et al 2015 Perovskite thin films via atomic layer deposition Adv. Mater. 27 53-58

[191] Chua S J, Lim S H, Kanhere R and Tua G F 1991 Interdigitated contact semiconductor laser as optoelectronic and gate Electron. Lett. 27 1963-4

[192] Chua S J and Li S H 2005 Optically triggered light sources with interdigitated contact semiconductor lasers CLEO/Pacific Rim'95. The Pacific Rim Conf. on Lasers and Electro-Optics (IEEE) p 191

[193] Chua S J 1996 Smart lasers Semiconductor Devices ed K Lal (New Delhi, India: Narosa Publishing House) pp 162-8

[194] Guo Y, Alkhazragi O, Kang C H, Shen C, Mao Y, Sun X, Ng T K and Ooi B S 2019 A tutorial on laser-based lighting and visible light communications: device and technology Chin. Opt. Lett. 17040601

[195] Zafar F, Bakaul M and Parthiban R 2017 Laser-diode-based visible light communication: toward gigabit class communication IEEE Commun. Mag. 55 144-51

[196] Melanson J E and Lucy C A 2000 Violet (405 nm) diode laser for laser induced fluorescence detection in capillary electrophoresis Analyst 125 1049-52

[197] Girkin J, Ferguson A, Wokosin D and Gurney A 2000 Confocal microscopy using an InGaN violet laser diode at 406nm Opt. Express 7336
[198] Ooi A, Wong A, Ng T K, Marondedze C, Gehring C and Ooi B S 2016 Growth and development of Arabidopsis thaliana under single-wavelength red and blue laser light Sci. Rep. 633885

[199] Komori K, Takasu Y, Kumakura M, Takahashi Y and Yabuzaki T 2003 Injection-locking of blue laser diodes and its application to the laser cooling of neutral ytterbium atoms Japan. J. Appl. Phys. 42 5059-62

[200] Burns I S, Hult J and Kaminski C F 2004 Spectroscopic use of a novel blue diode laser in a wavelength region around $450 \mathrm{~nm}$ Appl. Phys. B 79 491-5

[201] Coldren L A 2000 Monolithic tunable diode lasers IEEE J. Sel. Top. Quantum Electron. 6 988-99

[202] Marsh J H and Hou L 2017 Mode-locked laser diodes and their monolithic integration IEEE J. Sel. Top. Quantum Electron. 231100611

[203] Miyajima T, Watanabe H, Ikeda M and Yokoyama H 2009 Picosecond optical pulse generation from self-pulsating bisectional GaN-based blue-violet laser diodes Appl. Phys. Lett. 94161103

[204] Kafar A, Stańczyk S, Grzanka S, Czernecki R, Leszczyński M, Suski T and Perlin P 2012 Cavity suppression in nitride based superluminescent diodes J. Appl. Phys. 111083106

[205] Olle V F, Vasil'ev P P, Wonfor A, Penty R V and White I H 2012 Ultrashort superradiant pulse generation from a $\mathrm{GaN} / \mathrm{InGaN}$ heterostructure Opt. Express 207035

[206] Shen C, Ng T K, Lee C, Nakamura S, Speck J S, DenBaars S P, Alyamani A Y, El-Desouki M M and Ooi B S 2018 Semipolar InGaN quantum-well laser diode with integrated amplifier for visible light communications $O p t$. Express 26 A219

[207] Holc K, Weig T, Köhler K, Wagner J and Schwarz U T 2013 Impact of band structure and absorber dynamics on self-Q-switching in GaN-based multisection laser diodes at high reverse bias Appl. Phys. Express 6084101

[208] Vasil'ev P P et al 2013 Mode locking in monolithic two-section InGaN blue-violet semiconductor lasers Appl. Phys. Lett. 102121115

[209] Shen C et al 2016 High-modulation-efficiency, integrated waveguide modulator-laser diode at $448 \mathrm{~nm} \mathrm{ACS}$ Photonics $3262-8$

[210] Watanabe H, Kuramoto M, Kono S, Ikeda M and Yokoyama H 2010 Blue-violet bow-tie self-pulsating laser diode with a peak power of $20 \mathrm{~W}$ and a pulse energy of 310 pJ Appl. Phys. Express 3122103

[211] Feng M et al 2018 On-chip integration of GaN-based laser, modulator, and photodetector grown on Si IEEE J. Sel. Top. Quantum Electron. 248200305

[212] Stanczyk S, Kafar A, Suski T, Wisniewski P, Czernecki R, Leszczynski M, Zajac M and Perlin P 2012 InGaN tapered laser diodes Electron. Lett. 481232

[213] Dumitru V, Schweizer H, Graäbeldinger H, Haärle R, Bader S, Bruürderl G, Weimar A, Lell A and Haärle V 2003 InGaN/GaN multi-quantum well distributed Bragg reflector laser diode with second-order gratings Electron. Lett. 39372

[214] Marinelli C, Sargent L J, Bordovsky M, Wonfor A, Rorison J M, Penty R V, White I H, Heard P J, Hasnain G and Schneider R P Jr 2001 Enhanced-performance operation of InGaN MQW lasers with air/nitride-distributed Bragg reflector defined by focused ion beam etching Proc. SPIE 428757

[215] Kneissl M, Paoli T L, Kiesel P, Treat D W, Teepe M, Miyashita N and Johnson N M 2002 Two-section InGaN multiple-quantum-well laser diode with integrated electroabsorption modulator Appl. Phys. Lett. 80 3283-5 
[216] Shen C, Lee C, Ng T K, Speck J S, Nakamura S, DenBaars S P, Alyamani A Y, Eldesouki M M and Ooi B S $2016 \mathrm{GHz}$ modulation enabled using large extinction ratio waveguide-modulator integrated with $404 \mathrm{~nm} \mathrm{GaN}$ laser diode 2016 IEEE Photonics Conf. (IPC) pp 813-4

[217] Monavarian M, Rashidi A, Aragon A A, Oh S H, Rishinaramangalam A K, DenBaars S P and Feezell D 2018 Impact of crystal orientation on the modulation bandwidth of InGaN/GaN light-emitting diodes Appl. Phys. Lett. 112041104

[218] Sari E, Nizamoglu S, Ozel T and Demir H V 2007 Blue quantum electroabsorption modulators based on reversed quantum confined Stark effect with blueshift Appl. Phys. Lett. 9011101

[219] Aoki M, Suzuki M, Sano H, Kawano T, Ido T, Taniwatari T, Uomi K and Takai A 1993 InGaAs/InGaAsP MQW electroabsorption modulator integrated with a DFB laser fabricated by band-gap energy control selective area MOCVD IEEE J. Quantum Electron. 29 2088-96

[220] Scheibenzuber W G, Hornuss C, Schwarz U T, Sulmoni L, Dorsaz J, Carlin J F and Grandjean N 2011 Self-pulsation at zero absorber bias in $\mathrm{GaN}$-based multisection laser diodes Appl. Phys. Express 4062702

[221] Holc K, Weig T, Pletschen W, Köhler K, Wagner J and Schwarz U T 2013 Picosecond pulse generation in monolithic GaN-based multi-section laser diodes Proc. SPIE 8625862515

[222] Tronciu V Z, Yamada M, Kawakami T, Ito S, Ohno T, Taneya M and Abram R A 2004 A theoretical and experimental investigation of the dynamics of tandem blue-violet lasers Opt. Commun. 235 409-14

[223] Oki T, Saito K, Watanabe H, Miyajima T, Kuramoto M, Ikeda M and Yokoyama H 2010 Passive and hybrid mode-locking of an external-cavity GaInN laser diode incorporating a strong saturable absorber Appl. Phys. Express 3032104

[224] Miyajima T, Kono S, Watanabe H, Oki T, Koda R, Kuramoto M, Ikeda M and Yokoyama H 2011 Saturable absorbing dynamics of GaInN multiquantum well structures Appl. Phys. Lett. 98171904

[225] Kawaguchi Y, Tani Y, Vaccaro P O, Ito S and Kawanishi H 2011 Electric field induced carrier sweep-out in tandem InGaN multi-quantum-well self-pulsating laser diodes Japan. J. Appl. Phys. $\mathbf{5 0} 020209$

[226] Kono S, Watanabe H, Koda R, Miyajima T and Kuramoto M 2012 200-fs pulse generation from a GaInN semiconductor laser diode passively mode-locked in a dispersion-compensated external cavity Appl. Phys. Lett. 101081121

[227] Koda R, Oki T, Kono S, Miyajima T, Watanabe H, Kuramoto M, Ikeda M and Yokoyama H 2012300 W peak power picosecond optical pulse generation by blue-violet GaInN mode-locked laser diode and semiconductor optical amplifier Appl. Phys. Express 5022702

[228] Sulmoni L, Lamy J M, Dorsaz J, Castiglia A, Carlin J F, Scheibenzuber W G, Schwarz U T, Zeng X, Boïko D L and Grandjean N 2012 Static and dynamic properties of multi-section InGaN-based laser diodes J. Appl. Phys. 112103112

[229] Olle V F, Wonfor A, Sulmoni L A M, Vasilèv P P, Lamy J M, Carlin J F, Grandjean N, Penty R V and White I H 2013 Hybrid and passive mode-locking of a monolithic two-section MQW InGaN/GaN laser diode IEEE Photonics Technol. Lett. 25 1514-6

[230] Weig T, Schwarz U T, Sulmoni L, Lamy J-M, Carlin J-F, Grandjean N and Boiko D 2013 Passive mode-locking in the cavity of monolithic GaN-based multi-section laser diodes Proc. SPIE $864086400 \mathrm{H}$
[231] Holc K, Lükens G, Weig T, Köhler K, Wagner J and Schwarz U T 2014 Gallium nitride laser diodes with integrated absorber: on the dynamics of self-pulsation Phys. Status Solidi 11 670-3

[232] Weig T, Lükens G, Holc K, Köhler K, Wagner J and Schwarz U T 2014 Absorption at large reverse bias in monolithic GaN-based short-pulse-multi-section laser diodes Proc. SPIE 9002 90020K

[233] Zeng X, Sulmoni L, Lamy J M, Stadelmann T, Grossmann S, Hoogerwerf A C, Grandjean N and Boïko D L 2015 Solitary pulse-on-demand production by optical injection locking of passively Q-switched InGaN diode laser near lasing threshold Appl. Phys. Lett. 106071101

[234] Weig T, Höck H, Holc K, Köhler K, Wagner J and Schwarz U T 2015 Implementation and investigation of mode locking in GaN-based laser diodes in external cavity configuration Phys. Status Solidi a 212 986-91

[235] Koda R, Takiguchi Y, Kono S, Watanabe H, Hanzawa Y, Nakajima H, Shiozaki M, Sugawara N, Kuramoto M and Narui H 2015 Generation of a $2.2 \mathrm{~nJ}$ picosecond optical pulse with blue-violet wavelength using a GaInN master oscillator power amplifier Appl. Phys. Lett. 107041116

[236] Kono S, Koda R, Kawanishi H and Narui H 2017 9-kW peak power and 150 -fs duration blue-violet optical pulses generated by GaInN master oscillator power amplifier Opt. Express 2514926

[237] Scheibenzuber W G, Schwarz U T, Sulmoni L, Carlin J F, Castiglia A and Grandjean N 2010 Bias-dependent absorption coefficient of the absorber section in GaN-based multisection laser diodes Appl. Phys. Lett. 97181103

[238] Koda R, Oki T, Miyajima T, Watanabe H, Kuramoto M, Ikeda M and Yokoyama H $2010100 \mathrm{~W}$ peak-power $1 \mathrm{GHz}$ repetition picoseconds optical pulse generation using blue-violet GaInN diode laser mode-locked oscillator and optical amplifier Appl. Phys. Lett. 97021101

[239] Saito K, Watanabe H, Miyajima T, Ikeda M and Yokoyama H 2010 Mode locking of an external-cavity bisection GaInN blue-violet laser diode producing 3 ps duration optical pulses Appl. Phys. Lett. 96031112

[240] Watanabe H, Miyajima T, Kuramoto M, Ikeda M and Yokoyama H 2010 10-W peak-power picosecond optical pulse generation from a triple section blue-violet self-pulsating laser diode Appl. Phys. Express 3052701

[241] Tsang D Z and Walpole J N 1983 Q-switched semiconductor diode lasers IEEE J. Quantum Electron. 19 145-56

[242] Jones D J, Zhang L M, Carroll J E and Marcenac D D 1995 Dynamics of monolithic passively mode-locked semiconductor lasers IEEE J. Quantum Electron. 31 1051-8

[243] Olle V, Vasil'ev P P, Wonfor A, Penty R and White I 2012 1.4 ps superradiant pulses from a GaN-based laser Conf. on Lasers and Electro-Optics 2012 (Washington, DC) p QM1G.2

[244] Boiko D L and Vasil'ev P P 2012 Superradiance dynamics in semiconductor laser diode structures Opt. Express 20 A9501

[245] White I H, Vasil'ev P P and Penty R V 2012 Ultrashort pulse generation in diode laser devices 2012 IEEE Photonics Conf. (IEEE) pp 396-7

[246] Boiko D L, Zeng X, Stadelmann T, Grossmann S, Hoogerwerf A, Weig T, Schwarz U T, Sulmoni L, Lamy J-M and Grandjean N 2013 Superfluorescent emission in electrically pumped semiconductor laser (arXiv:1302.0263) pp 1-19

[247] Boiko D L, Zeng X, Stadelmann T, Grossmann S and Hoogerwerf A C 2014 Superradiance in electrically pumped semiconductor laser: myth or reality? 2014 Int. Semiconductor Laser Conf. (IEEE) pp 221-2 
[248] Shen C, Ng T K, Leonard J T, Pourhashemi A, Nakamura S, DenBaars S P, Speck J S, Alyamani A Y, El-desouki M M and Ooi B S 2016 High-brightness semipolar $\left(2021^{-}\right)$blue InGaN/GaN superluminescent diodes for droop-free solid-state lighting and visible-light communications $O p t$. Lett. 41 2608-11

[249] Goldberg G R, Ivanov P, Ozaki N, Childs D T D, Groom K M, Kennedy K L and Hogg R A 2017 Gallium nitride light sources for optical coherence tomography Proc. SPIE $10104101041 \mathrm{X}$

[250] Goldberg G R, Boldin A, Andersson S M L, Ivanov P, Ozaki N, Taylor R J E, Childs D T D, Groom K M, Kennedy K L and Hogg R A 2017 Gallium nitride superluminescent light emitting diodes for optical coherence tomography applications IEEE J. Sel. Top. Quantum Electron. 232000511

[251] Shen C, Ng T K, Lee C, Leonard J T, Nakamura S, Speck J S, Denbaars S P, Alyamani A Y, El-Desouki M M and Ooi B S 2017 Semipolar InGaN-based superluminescent diodes for solid-state lighting and visible light communications Proc. SPIE $10104101041 \mathrm{U}$

[252] Eisenstein G 1989 Semiconductor optical amplifiers IEEE Circuits Devices Mag. 5 25-30

[253] Andreeva E V, Anikeev A S, Il'chenko S N, Lobintsov A A, Chamorovskii A Y, Shidlovskii V R, Shramenko M V and Yakubovich S D 2019 Tunable laser based on a semiconductor optical amplifier of red spectral region Quantum Electron. 49 493-6

[254] Blumenthal D J 2020 Photonic integration for UV to IR applications APL Photon. 520903

[255] Stanczyk S, Kafar A, Grzanka S, Sarzynski M, Mroczynski R, Najda S, Suski T and Perlin P 2018450 $\mathrm{nm}(\mathrm{Al}, \mathrm{In}) \mathrm{GaN}$ optical amplifier with double ' $\mathrm{j}$-shape' waveguide for master oscillator power amplifier systems Opt. Express 267351

[256] Kafar A, Stanczyk S, Schiavon D, Suski T and Perlin P 2020 Review-review on optimization and current status of (Al,In)GaN superluminescent diodes ECS J. Solid State Sci. Technol. 9015010

[257] Shen C, Lee C, Stegenburgs E, Lerma J H, Ng T K, Nakamura S, DenBaars S P, Alyamani A Y, El-Desouki M M and Ooi B S 2017 Semipolar III-nitride quantum well waveguide photodetector integrated with laser diode for on-chip photonic system Appl. Phys. Express 10042201

[258] Zhang J et al 2019 III-V-on-Si photonic integrated circuits realized using micro-transfer-printing APL Photon. 4110803

[259] Cho J, Cho S, Kim B J, Chae S, Sone C, Nam O H, Lee J W, Park Y and Kim T I 2000 InGaN/GaN multi-quantum well distributed Bragg reflector laser diode Appl. Phys. Lett. 76 1489-91

[260] Marinelli C et al 2001 Design and performance analysis of deep-etch air/nitride distributed Bragg reflector gratings for AlInGaN laser diodes Appl. Phys. Lett. 79 4076-8

[261] Wang H, Kumagai M, Tawara T, Nishida T, Akasaka T, Kobayashi N and Saitoh T 2002 Fabrication of an InGaN multiple-quantum-well laser diode featuring high reflectivity semiconductor/air distributed Bragg reflectors Appl. Phys. Lett. 81 4703-5

[262] Ren Q, Zhang B, Zhang B, Xu J, Yang Z J and Hu X D 2004 Micro-zone optical measurements on $\mathrm{GaN}$ based nitride/air distributed Bragg reflector (DBR) mirrors made by focused ion beam milling Phys. Status Solidi c $12450-3$

[263] Dai T et al 2007 Focused ion beam etched nitride/air DBRs as cavity mirror facets of violet InGaN/GaN multiplequantum well laser diodes Phys. Status Solidi c 4 196-9
[264] Xie W, Li J, Liao M, Deng Z, Wang W and Sun S 2019 Narrow linewidth distributed Bragg reflectors based on InGaN/GaN laser Micromachines 10529

[265] Sanz D C, Rorison J and Yu S 2005 InGaN/GaN MQW laser diodes with 4th order FIB-etched gratings 2005 Quantum Electronics and Laser Science Conf. vol 2 (IEEE) pp $1023-5$

[266] Nakamura S, Senoh M, Nagahama S, Iwasa N, Yamada T, Matsushita T, Kiyoku H and Sugimoto Y 1996 InGaN-based multi-quantum-well-structure laser diodes Japan. J. Appl. Phys. 35 L74-6

[267] Carroll J, Whiteaway J and Plumb D 1998 Distributed Feedback Semiconductor Lasers (London: IEE-SPIE)

[268] Masui S, Tsukayama K, Yanamoto T, Kozaki T, Nagahama S and Mukai T $2006 \mathrm{CW}$ operation of the first-order AlInGaN $405 \mathrm{~nm}$ distributed feedback laser diodes Japan. J. Appl. Phys. 45 L1223-5

[269] Kang J H et al 2020 Continuous-wave operation of DFB laser diodes based on $\mathrm{GaN}$ using 10th-order laterally coupled surface gratings Opt. Lett. 45935

[270] Schweizer H, Gräbeldinger H, Dumitru V, Jetter M, Bader S, Brüderl G, Weimar A, Lell A and Härle V 2002 Laterally coupled InGaN/GaN DFB laser diodes Phys. Status Solidi a 192 301-7

[271] Masui S, Tsukayama K, Yanamoto T, Kozaki T, Nagahama S-I and Mukai T 2008 Characterization of AlInGaN-based $405 \mathrm{~nm}$ distributed feedback laser diodes Proc. SPIE 6909 69090G

[272] Masui S, Tsukayama K, Yanamoto T, Kozaki T, Nagahama S and Mukai T 2006 First-order AlInGaN 405 nm distributed feedback laser diodes by current injection Japan. J. Appl. Phys. 45 L749-51

[273] Slight T J, Odedina O, Meredith W, Docherty K E and Kelly A E 2016 InGaN/GaN distributed feedback laser diodes with deeply etched sidewall gratings IEEE Photonics Technol. Lett. 28 2886-8

[274] Slight T J, Odedina O, Meredith W, Docherty K E and Kelly A E 2016 InGaN/GaN DFB laser diodes at $434 \mathrm{~nm}$ with deeply etched sidewall gratings Proc. SPIE 9748 97481A

[275] Slight T J, Yadav A, Odedina O, Meredith W, Docherty K E, Rafailov E and Kelly A E 2017 InGaN/GaN laser diodes with high order notched gratings IEEE Photonics Technol. Lett. 29 2020-2

[276] Kang J H, Martens M, Wenzel H, Hoffmann V, John W, Einfeldt S, Wernicke T and Kneiss1 M 2017 Optically pumped DFB lasers based on GaN using 10th-order laterally coupled surface gratings IEEE Photonics Technol. Lett. 29 138-41

[277] Najda S P et al 2017 Lateral grating DFB AlGaInN laser diodes for optical communications and atomic clocks XXIII Int. Conf. Spectr. Line Shapes vol 810 p 012053

[278] Slight T J et al 2018 Continuous-wave operation of (Al,In)GaN distributed-feedback laser diodes with high-order notched gratings Appl. Phys. Express 11112701

[279] Kang J H, Wenzel H, Hoffmann V, Freier E, Sulmoni L, Einfeldt S, Wernicke T, Kneissl M and Unger R-S 2018 10th order laterally coupled GaN-based DFB laser diodes with V-shaped surface gratings Proc. SPIE 10553 105530A

[280] Hofmann R et al 1996 Realization of optically pumped second-order GaInN-distributed-feedback lasers Appl. Phys. Lett. $692068-70$

[281] Kang J H, Wenzel H, Hoffmann V, Freier E, Sulmoni L, Unger R-S, Einfeldt S, Wernicke T and Kneissl M 2018 DFB laser diodes based on GaN using 10th order laterally coupled surface gratings IEEE Photonics Technol. Lett. 30 231-4 
[282] Zhang H, Cohen D A, Chan P, Wong M S, Mehari S, Becerra D L, Nakamura S and DenBaars S P 2019 Continuous-wave operation of a semipolar InGaN distributed-feedback blue laser diode with a first-order indium tin oxide surface grating Opt. Lett. 443106

[283] Deng Z, Li J, Liao M, Xie W and Luo S 2019 InGaN/GaN distributed feedback laser diodes with surface gratings and sidewall gratings Micromachines 10699

[284] Watson S et al 2019 Applications of single frequency blue lasers 2019 21st Int. Conf. on Transparent Optical Networks (ICTON) (Piscataway, NJ: IEEE) pp 1-4

[285] Gwyn S et al 2019 GaN-based distributed feedback laser diodes for optical communications Proc. SPIE 112071120700

[286] Zhang H, Cohen D, Chan P, Wong M, Mehari S, Nakamura S and DenBaars S 2020 Semipolar group III-nitride distributed-feedback blue laser diode with Indium tin oxide surface grating Proc. SPIE 113011130102

[287] Holguín-Lerma J A, Ng T K and Ooi B S 2019 Narrow-line InGaN/GaN green laser diode with high-order distributed-feedback surface grating Appl. Phys. Express 12042007

[288] Holguin-Lerma J A, Kong M, Alkhazragi O, Sun X, Ng T K and Ooi B S 2020 480-nm distributed-feedback InGaN laser diode for 10.5-Gbit/s visible-light communication Opt. Lett. 45742

[289] Hofmann R, Wagner V, Gauggel H P, Adler F, Ernst P, Sohmer A, Bolay H, Scholz F and Schweizer H 1997 Realization and characterization of optically pumped GaInN-DFB lasers IEEE J. Sel. Top. Quantum Electron. 3 456-60

[290] Hofstetter D, Thornton R L, Romano L T, Bour D P, Kneissl M and Donaldson R M 1998 Room-temperature pulsed operation of an electrically injected InGaN/GaN multi-quantum well distributed feedback laser Appl. Phys. Lett. 73 2158-60

[291] Abare A C, Hansen M, Speck J S, DenBaars S P and Coldren L A 1999 Electrically pumped distributed feedback nitride lasers employing embedded deielectric gratings Electron. Lett. 35 1559-60

[292] Hofmann R, Wagner V, Neuner M, Off J, Scholz F and Schweizer H 1999 Optically pumped GaInN/GaN-DFB lasers: overgrown lasers and vertical modes Mater. Sci. Eng. B 59 386-9

[293] Werner R, Reinhardt M, Emmerling M, Forchel A, Härle V and Bazhenov A 2000 High-resolution patterning and characterization of optically pumped first-order GaN DFB lasers Physica E 7 915-8

[294] Hofstetter D, Romano L T, Paoli T L, Bour D P and Kneissl M 2000 Realization of a complex-coupled InGaN/GaN-based optically pumped multiple-quantumwell distributed-feedback laser Appl. Phys. Lett. 76 2337-9

[295] Abare A C, Denbaars S P and Coldren L A 2000 Distributed feedback laser diodes employing embedded dielectric gratings located above the active region IEICE Trans. Electron. E83-C 560-3

[296] Holguín-Lerma J A, Ng T K and Ooi B S 2020 Off-the-shelf laser diodes with narrow-line emission at visible wavelengths by integration of distributed feedback (DFB) surface grating (conference presentation) Proc. SPIE 113011130104

[297] Poli N, Oates C W, Gill P and Tino G M 2013 Optical atomic clocks Riv. del Nuovo Cim. 1 555-624

[298] Kong M, Holguin-Lerma J A, Alkhazragi O, Sun X, Ng T K and Ooi B S 2020 10-Gbit/s sky-blue distributed feedback laser diode-based visible light communication 2020
Optical Fiber Communications Conf. and Exhibition, OFC 2020_Proc. (Piscataway, NJ: IEEE) p T3C.3

[299] Matuschek N and Duelk M 2017 Superluminescent light-emitting diodes Handbook of Optoelectronic Device Modeling and Simulation (Boca Raton, FL: CRC Press) pp 589-608

[300] Alatawi A A, Holguin-Lerma J A, Shen C, Shakfa M K, Alhamoud A A, Albadri A M, Alyamani A Y, Ng T K and Ooi B S 2018 High power GaN-based blue superluminescent diode exceeding $450 \mathrm{~mW} 2018$ IEEE Int. Semiconductor Laser Conf. (ISLC) (Piscataway, NJ: IEEE) pp 129-30

[301] Marco R, Julien D, Raffaele R, Marcus D, Christian V, Eric F, Antonino C, Gatien C, Jean-François C and Nicolas G 2010 High power blue-violet superluminescent light emitting diodes with InGaN quantum wells Appl. Phys. Express 361002

[302] Kopp F, Eichler C, Lell A, Tautz S, Ristić J, Stojetz B, Höß C, Weig T, Schwarz U T and Strauss U 2013 Blue superluminescent light-emitting diodes with output power above $100 \mathrm{~mW}$ for picoprojection Japan. J. Appl. Phys. 52 08JH07

[303] Hardy M T, Kelchner K M, Lin Y D, Hsu P S, Fujito K, Ohta H, Speck J S, Nakamura S and DenBaars S P 2009 $m$-plane GaN-based blue superluminescent diodes fabricated using selective chemical wet etching Appl. Phys. Express 2121004

[304] Fujimoto J G, Brezinski M E, Tearney G J, Boppart S A, Bouma B, Hee M R, Southern J F and Swanson E A 1995 Optical biopsy and imaging using optical coherence tomography Nat. Med. $1970-2$

[305] Böhm K, Marten P, Petermann K, Weidel E and Ulrich R 1981 Low-drift fibre gyro using a superluminescent diode Electron. Lett. 17 352-3

[306] Alphonse G A 2002 Design of high-power superluminescent diodes with low spectral modulation Proc. SPIE 4648 125-38

[307] Shen C, Holguin-Lerma J A, Alatawi A A, Zou P, Chi N, Ng T K and Ooi B S 2019 Group-III-nitride superluminescent diodes for solid-state lighting and high-speed visible light communications IEEE J. Sel. Top. Quantum Electron. 252000110

[308] Alatawi A A, Holguin-Lerma J A, Kang C H, Shen C, Subedi R C, Albadri A M, Alyamani A Y, Ng T K and Ooi B S 2018 High-power blue superluminescent diode for high CRI lighting and high-speed visible light communication Opt. Express 26 26355-64

[309] Feltin E et al 2009 Broadband blue superluminescent light-emitting diodes based on GaN Appl. Phys. Lett. 9581107

[310] Shen C, Lee C, Ng T K, Nakamura S, Speck J S, DenBaars S P, Alyamani A Y, El-Desouki M M and Ooi B S 2016 High-speed 405-nm superluminescent diode (SLD) with 807-MHz modulation bandwidth Opt. Express 24 20281-6

[311] Hu F, Holguín-Lerma J A, Mao Y, Shen C, Sun X, Kong M, Ng T K, Ooi B S and Chi N 2020 3.8-Gbit/s visible light communication (VLC) based on 443-nm superluminescent diode and bit-loading discrete-multiple-tone (DMT) modulation scheme Proc. SPIE $11307113070 \mathrm{H}$

[312] Kafar A, Stańczyk S, Targowski G, Czernecki R, Wiśniewski P, Leszczyński M, Suski T and Perlin P 2013 High optical power ultraviolet superluminescent InGaN diodes Proc. SPIE $\mathbf{8 6 2 5} 86251 \mathrm{~S}$

[313] Kafar A, Stanczyk S, Sarzynski M, Grzanka S, Goss J, Targowski G, Nowakowska-Siwinska A, Suski T and Perlin P 2016 Nitride superluminescent diodes with broadened emission spectrum fabricated using laterally patterned substrate Opt. Express 24 9673-82 
[314] Wang L, Wang L, Yu J, Hao Z, Luo Y, Sun C, Han Y, Xiong B, Wang J and Li H 2019 Abnormal stranski-krastanov mode growth of green InGaN quantum dots: morphology, optical properties, and applications in light-emitting devices ACS Appl. Mater. Interfaces 11 1228-38

[315] Castiglia A, Rossetti M, Matuschek N, Rezzonico R, Duelk M, Vélez C, Carlin J-F and Grandjean N 2016 GaN-based superluminescent diodes with long lifetime Proc. SPIE 9748 97481V

[316] Cahill R, Maaskant P P, Akhter M and Corbett B 2019 High power surface emitting InGaN superluminescent light-emitting diodes Appl. Phys. Lett. 115171102

[317] Rashidi A, Rishinaramangalam A K, Aragon A A, Mishkat-Ul-Masabih S, Monavarian M, Lee C, Denbaars S P and Feezell D F 2020 High-speed nonpolar InGaN/GaN superluminescent diode with $2.5 \mathrm{GHz}$ modulation bandwidth IEEE Photonics Technol. Lett. 32 383-6

[318] Zhang H, Shih C, Martin D, Caut A, Carlin J, Butté R and Grandjean N 2020 Broadened bandwidth amplified spontaneous emission from blue GaN-based short-cavity superluminescent light-emitting diodes ECS J. Solid State Sci. Technol. 9015019

[319] Liu J et al 2019 InGaN-based quantum well superluminescent diode monolithically grown on $\mathrm{Si} A C S$ Photonics 6 2104-9

[320] Kafar A, Stanczyk S, Sarzynski M, Grzanka S, Goss J, Makarowa I, Nowakowska-Siwinska A, Suski T and Perlin P 2017 InAlGaN superluminescent diodes fabricated on patterned substrates: an alternative semiconductor broadband emitter Photonics Res. 5 A30

[321] Primerov N et al 2019 A compact red-green-blue superluminescent diode module: a novel light source for AR microdisplays Proc. SPIE 11062 110620F

[322] Rossetti M, Castiglia A, Malinverni M, Mounir C, Matuschek N, Duelk M and Vélez C 2018 3-5: RGB superluminescent diodes for AR micro-displays SID Symp. Digest of Technical Papers vol 49 pp 17-20

[323] Piprek J 2019 What limits the efficiency of GaN-based superluminescent light-emitting diodes (SLEDs)? Opt. Quantum Electron. $\mathbf{5 1} 382$

[324] Harwell J R, Whitworth G L, Turnbull G A and Samuel I D W 2017 Green perovskite distributed feedback lasers Sci. Rep. 711727

[325] Frost T, Jahangir S, Stark E, Deshpande S, Hazari A, Zhao C, Ooi B S and Bhattacharya P 2014 Monolithic electrically injected nanowire array edge-emitting laser on (001) silicon Nano Lett. 14 4535-41

[326] Iga K 2018 Forty years of vertical-cavity surface-emitting laser: invention and innovation Japan. J. Appl. Phys. 57 08PA01

[327] Ra Y H, Rashid R T, Liu X, Sadaf S M, Mashooq K and Mi Z 2020 An electrically pumped surface-emitting semiconductor green laser $S c i . A d v .6$ eaav 7523

[328] Arafin S, Liu X and Mi Z 2013 Review of recent progress of III-nitride nanowire lasers J. Nanophoton. 7074599

[329] Li K H, Liu X, Wang Q, Zhao S and Mi Z 2015 Ultralow-threshold electrically injected AlGaN nanowire ultraviolet lasers on Si operating at low temperature Nat. Nanotechnol. 10 140-4

[330] Alias M S, Liu Z, Al-atawi A, Ng T K, Wu T and Ooi B S 2017 Continuous-wave optically pumped green perovskite vertical-cavity surface-emitter Opt. Lett. 42 3618-21

[331] Chen S and Nurmikko A 2017 Stable green perovskite vertical-cavity surface-emitting lasers on rigid and flexible substrates ACS Photonics 4 2486-94
[332] Chen S, Zhang C, Lee J, Han J and Nurmikko A 2017 High- $Q$, low-threshold monolithic perovskite thin-film vertical-cavity lasers Adv. Mater. 291604781

[333] Elafandy R T, Kang J H, Li B, Kim T K, Kwak J S and Han J 2020 Room-temperature operation of $c$-plane $\mathrm{GaN}$ vertical cavity surface emitting laser on conductive nanoporous distributed Bragg reflector Appl. Phys. Lett. 117011101

[334] Lim K T P, Deakin C, Ding B, Bai X, Griffin P, Zhu T, Oliver R A and Credgington D 2019 Encapsulation of methylammonium lead bromide perovskite in nanoporous GaN APL Mater. 7021107

[335] Demchyshyn S et al 2017 Confining metal-halide perovskites in nanoporous thin films Sci. Adv. 3 e 1700738

[336] De Giorgi M L and Anni M 2019 Amplified spontaneous emission and lasing in lead halide perovskites: state of the art and perspectives Appl. Sci. 94591

[337] Ou Q, Bao X, Zhang Y, Shao H, Xing G, Li X, Shao L and Bao Q 2019 Band structure engineering in metal halide perovskite nanostructures for optoelectronic applications Nano Mater. Sci. 1 268-87

[338] Fan J, Du W, Mi Y and Liu X 2019 Perovskite-based lasers Nanoscale Semiconductor Lasers (Amsterdam: Elsevier) pp 41-74

[339] Xing G, Mathews N, Lim S S, Yantara N, Liu X, Sabba D, Grätzel M, Mhaisalkar S and Sum T C 2014

Low-temperature solution-processed wavelength-tunable perovskites for lasing Nat. Mater. 13476

[340] Priante D, Dursun I, Alias M S, Shi D, Melnikov V A, Ng T K, Mohammed O F, Bakr O M and Ooi B S 2015 The recombination mechanisms leading to amplified spontaneous emission at the true-green wavelength in CH3NH3PbBr3 perovskites Appl. Phys. Lett. 10681902

[341] Li B, Fu L, Li S, Li H, Pan L, Wang L, Chang B and Yin L 2019 Pathways toward high-performance inorganic perovskite solar cells: challenges and strategies J. Mater. Chem. A 7 20494-518

[342] Wang Y, Zhi M, Chang Y-Q, Zhang J-P and Chan Y 2018 Stable, ultralow threshold amplified spontaneous emission from $\mathrm{CsPBr}_{3}$ nanoparticles exhibiting trion gain Nano Lett. 18 4976-84

[343] Wang S, Yu J, Zhang M, Chen D, Li C, Chen R, Jia G, Rogach A L and Yang X 2019 Stable, strongly emitting cesium lead bromide perovskite nanorods with high optical gain enabled by an intermediate monomer reservoir synthetic strategy Nano Lett. 19 6315-22

[344] Yan D, Shi T, Zang Z, Zhou T, Liu Z, Zhang Z, Du J, Leng Y and Tang $\mathrm{X} 2019$ Ultrastable $\mathrm{CsPbBr} 3$ perovskite quantum dot and their enhanced amplified spontaneous emission by surface ligand modification Small 151901173

[345] Huang C-Y, Zou C, Mao C, Corp K L, Yao Y-C, Lee Y-J, Schlenker C W, Jen A K Y and Lin L Y 2017 CsPbBr 3 perovskite quantum dot vertical cavity lasers with low threshold and high stability ACS Photonics 4 2281-9

[346] Gharajeh A, Haroldson R, Li Z, Moon J, Balachandran B, $\mathrm{Hu}$ W, Zakhidov A and Gu Q 2018 Continuous-wave operation in directly patterned perovskite distributed feedback light source at room temperature Opt. Lett. 43611

[347] Gunnarsson W B and Rand B P 2020 Electrically driven lasing in metal halide perovskites: challenges and outlook APL Mater. 8030902

[348] Wang Y, Li X, Zhao X, Xiao L, Zeng H and Sun H 2016 Nonlinear absorption and low-threshold multiphoton pumped stimulated emission from all-inorganic perovskite nanocrystals Nano Lett. 16 448-53 
[349] Zhang W, Peng L, Liu J, Tang A, Hu J-S, Yao J and Zhao Y S 2016 Controlling the cavity structures of two-photon-pumped perovskite microlasers $A d v$. Mater. 28 4040-6

[350] Yang B, Mao X, Yang S, Li Y, Wang Y, Wang M, Deng W and Han K 2016 Low threshold two-photon-pumped amplified spontaneous emission in $\mathrm{CH}_{3} \mathrm{NH}_{3} \mathrm{PbBr}_{3}$ microdisks ACS Appl. Mater. Interfaces 8 19587-92

[351] Tang $\mathrm{X}$ et al 2017 Perovskite $\mathrm{CsPb}_{2} \mathrm{Br}_{5}$ microplate laser with enhanced stability and tunable properties $A d v$. Opt. Mater. 51600788

[352] Arora N et al 2016 Photovoltaic and amplified spontaneous emission studies of high-quality formamidinium lead bromide perovskite films Adv. Funct. Mater. 26 2846-54

[353] Li C, Zang Z, Han C, Hu Z, Tang X, Du J, Leng Y and Sun K 2017 Highly compact $\mathrm{CsPbBr} 3$ perovskite thin films decorated by $\mathrm{ZnO}$ nanoparticles for enhanced random lasing Nano Energy 40 195-202

[354] De Giorgi M L, Perulli A, Yantara N, Boix P P and Anni M 2017 Amplified spontaneous emission properties of solution processed $\mathrm{CsPbBr}_{3}$ perovskite thin films J. Phys. Chem. C 121 14772-8

[355] Balena A, Perulli A, Fernandez M, De Giorgi M L, Nedelcu G, Kovalenko M V and Anni M 2018 Temperature dependence of the amplified spontaneous emission from $\mathrm{CsPBr}_{3}$ nanocrystal thin films J. Phys. Chem. C 122 5813-9

[356] Zhang L, Yuan F, Dong H, Jiao B, Zhang W, Hou X, Wang S, Gong Q and Wu Z 2018 One-step co-evaporation of all-inorganic perovskite thin films with room-temperature ultralow amplified spontaneous emission threshold and air stability ACS Appl. Mater. Interfaces 10 40661-71

[357] Zhao C, Tian W, Liu J, Sun Q, Luo J, Yuan H, Gai B, Tang J, Guo J and Jin S 2019 Stable two-photon pumped amplified spontaneous emission from millimeter-sized $\mathrm{CsPbBr}_{3}$ single crystals J. Phys. Chem. Lett. $102357-62$

[358] He H, Ma E, Chen X, Yang D, Chen B and Qian G 2019 Single crystal perovskite microplate for high-order multiphoton excitation Small Methods 31900396

[359] Liu Z, Hu Z, Shi T, Du J, Yang J, Zhang Z, Tang X and Leng Y 2019 Stable and enhanced frequency up-converted lasing from $\mathrm{CsPbBr}_{3}$ quantum dots embedded in silica sphere Opt. Express 279459

[360] Alias M S et al 2018 Review of nanophotonics approaches using nanostructures and nanofabrication for III-nitrides ultraviolet-photonic devices J. Nanophoton. 12043508

[361] Sutherland B R and Sargent E H 2016 Perovskite photonic sources Nat. Photonics 10 295-302

[362] Samuel I D W and Turnbull G A 2007 Organic semiconductor lasers Chem. Rev. 107 1272-95

[363] Brenner P, Bar-On O, Jakoby M, Allegro I, Richards B S, Paetzold U W, Howard I A, Scheuer J and Lemmer U 2019 Continuous wave amplified spontaneous emission in phase-stable lead halide perovskites Nat. Commun. 10988

[364] Suematsu Y 2014 Dynamic single-mode lasers J. Light Technol. 32 1144-58

[365] Ludlow A D, Boyd M M, Ye J, Peik E and Schmidt P O 2015 Optical atomic clocks Rev. Mod. Phys. 87637

[366] Antończak A J, Kozioł P, Sotor J Z, Kaczmarek P R and Abramski K M 2011 Laser Doppler vibrometry with a single-frequency microchip green laser Meas. Sci. Technol. 22115306

[367] Chen S, Roh K, Lee J, Chong W K, Lu Y, Mathews N, Sum T C and Nurmikko A 2016 A photonic crystal laser from solution based organo-lead iodide perovskite thin films ACS Nano 10 3959-67

[368] Doi A, Oketani R and Nawa Y 2018 High-resolution imaging in two-photon excitation microscopy using in situ estimations of the point spread function Biomed. Opt. Express 9202

[369] Cumpston B H et al 1999 Two-photon polymerization initiators for three-dimensional optical data storage and microfabrication Nature 398 51-54

[370] Makarov N S, Rebane A, Drobizhev M, Wolleb H and Spahni H 2007 Optimizing two-photon absorption for volumetric optical data storage J. Opt. Soc. Am. B 241874

[371] Lin H, Fan T, Sui J, Wang G, Chen J, Zhuo S and Zhang H 2019 Recent advances in multiphoton microscopy combined with nanomaterials in the field of disease evolution and clinical applications to liver cancer Nanoscale 11 19619-35

[372] Maestro L M, Rodriguez E M, Vetrone F, Naccache R, Ramirez H L, Jaque D, Capobianco J A and Solé J G 2010 Nanoparticles for highly efficient multiphoton fluorescence bioimaging Opt. Express 1823544

[373] Velusamy M et al 2009 A new series of quadrupolar type two-photon absorption chromophores bearing 11, 12-dibutoxydibenzo[ $[a, c]$-phenazine bridged amines; their applications in two-photon fluorescence imaging and two-photon photodynamic therapy Adv. Funct. Mater. 19 2388-97

[374] Kuebler S M, Xia C, Sharma R, Digaum J L, Martinez N P, Valle C L and Rumpf R C 2019 Fabrication of functional nanophotonic devices by multiphoton lithography Proc. SPIE 109151091502

[375] Pernice W H P, Xiong C, Schuck C and Tang H X 2012Second harmonic generation in phase matched aluminum nitride waveguides and micro-ring resonators Appl. Phys. Lett. 100223501

[376] Chen H, Fu H, Huang X, Zhang X, Yang T-H, Montes J A, Baranowski I and Zhao Y 2017 Low loss GaN waveguides at the visible spectral wavelengths for integrated photonics applications Opt. Express 25 31758-73

[377] Zhu H, Fu Y, Meng F, Wu X, Gong Z, Ding Q, Gustafsson M V, Trinh M T, Jin S and Zhu X Y 2015 Lead halide perovskite nanowire lasers with low lasing thresholds and high quality factors Nat. Mater. 14 636-42

[378] Wang D, Zhu T, Oliver R A and Hu E L 2018 Ultra-low-threshold InGaN/GaN quantum dot micro-ring lasers Opt. Lett. 43799

[379] Prabaswara A, Min J W, Zhao C, Janjua B, Zhang D, Albadri A M, Alyamani A Y, Ng T K and Ooi B S 2018 Direct growth of III-nitride nanowire-based yellow light-emitting diode on amorphous quartz using thin $\mathrm{Ti}$ interlayer Nanoscale Res. Lett. 1341

[380] Ratnesh R K and Mehata M S 2017 Investigation of biocompatible and protein sensitive highly luminescent quantum dots/nanocrystals of CdSe, CdSe/ZnS and CdSe/CdS Spectrochim. Acta A 179 201-10

[381] Saikia D, Chakravarty S, Sarma N S, Bhattacharjee S, Datta P and Adhikary N C 2017 Aqueous synthesis of highly stable CdTe/ZnS Core/Shell quantum dots for bioimaging Luminescence 32 401-8

[382] Extance A 2019 The reality behind solar power's next star material Nature 570 429-32

[383] Alias M S, Yang Y, Ng T K, Dursun I, Shi D, Saidaminov M I, Priante D, Bakr O M and Ooi B S 2016 Enhanced etching, surface damage recovery, and submicron patterning of hybrid perovskites using a chemically gas-assisted focused-ion beam for subwavelength grating photonic applications J. Phys. Chem. Lett. 7 137-42

[384] Fu H 2019 Review of lead-free halide perovskites as light-absorbers for photovoltaic applications: from materials to solar cells Sol. Energy Mater. Sol. Cells 193 107-32 
[385] Wang X, Zhang T, Lou Y and Zhao Y 2019 All-inorganic lead-free perovskites for optoelectronic applications Mater. Chem. Front. 3 365-75

[386] Xiao Z, Song Z and Yan Y 2019 From lead halide perovskites to lead-free metal halide perovskites and perovskite derivatives Adv. Mater. 311803792

[387] Dursun I et al 2016 Perovskite nanocrystals as a color converter for visible light communication ACS Photonics 3 1150-6

[388] Anon 2017 Scrutinizing lasers Nat. Photonics 11139

[389] Khenkin M V et al 2020 Consensus statement for stability assessment and reporting for perovskite photovoltaics based on ISOS procedures Nat. Energy 5 35-49

[390] Lee S, Forman C A, Kearns J, Leonard J T, Cohen D A, Nakamura S and DenBaars S P 2019 Demonstration of GaN-based vertical-cavity surface-emitting lasers with buried tunnel junction contacts Opt. Express 2731621

[391] Mishkat-Ul-Masabih S M, Luk T S, Monavarian M and Feezell D F 2019 Polarization-pinned emission of a continuous-wave optically pumped nonpolar GaN-based VCSEL using nanoporous distributed Bragg reflectors Opt. Express 279495
[392] Kamei T, Kamikawa T, Araki M, DenBaars S P, Nakamura S and Bowers J E 2020 Research toward a heterogeneously integrated InGaN laser on silicon Phys. Status Solidi a 2171900770

[393] Geuzebroek D, Dekker R and van Dijk P 2017 Photonics packaging made visible Opt. Photonik 12 34-38

[394] Arefin R, Ramachandra S H, Jung H, You W, Hasan S M N, Turski H, Dwivedi S and Arafin S 2020 III-N/SiN integrated photonics platform for blue wavelengths IEEE J. Quantum Electron. 566300309

[395] Chul Shin M et al 2020 Chip-scale blue light phased array Opt. Lett. 451934

[396] Sacher W D et al 2019 Visible-light silicon nitride waveguide devices and implantable neurophotonic probes on thinned $200 \mathrm{~mm}$ silicon wafers Opt. Express 2737400

[397] Duan Z, Yuan Y, Lu J C, Wang J L, Li Y, Svanberg S and Zhao G Y 2020 Underwater spatially, spectrally, and temporally resolved optical monitoring of aquatic fauna Opt. Express 282600

[398] West G N, Loh W, Kharas D, Sorace-Agaskar C, Mehta K K, Sage J, Chiaverini J and Ram R J 2019 Low-loss integrated photonics for the blue and ultraviolet regime APL Photonics 4026101 